\title{
Álgebras algébricas absolutamente valuadas
}

Eddie Arrieta Arrieta

DisSERTAÇÃO APRESENTADA

$\mathrm{AO}$

Instituto DE MATEMÁtica E EstatísticA

DA

Universidade DE SÃo PAUlo

PARA

OBTENÇÃO DO TÍTULO

$\mathrm{DE}$

Mestre EM CiÊNCIAS

Programa: Matemática

Orientador: Prof. Dr. Juan Carlos Gutiérrez Fernández

Durante o desenvolvimento deste trabalho o autor recebeu auxílio financeiro da CNPq

São Paulo, outubro de 2012 


\section{Álgebras algébricas absolutamente valudas}

Esta versão da dissertação contém as correções e alterações sugeridas pela Comissão Julgadora durante a defesa da versão original do trabalho, realizada em 14/11/2012. Uma cópia da versão original está disponível no

Instituto de Matemática e Estatística da Universidade de São Paulo.

Comissão Julgadora:

- Prof. Dr. Juan Carlos Gutiérrez Fernández (orientador) - IME-USP

- Prof. Dr. Henrique Guzzo Junior - IME-USP

- Prof. Dr. Plamen Emilov Kochloukov - Unicamp 
À genitora grande e ao velho de marcha devagar. 


\section{Agradecimentos}

Como pessoa e como estudante, sou especialmente grato com o professor Juan Carlos Gutiérrez Fernández, o meu orientador, por sua paciência e importantes ajudas com as quais foi possível a elaboração de esta dissertação.

Como cidadão, minha gratidão com esta República, o Brasil, por me permitir a utilização dos recursos da CNPq através do Instituto de Matemática e Estatística. Como homem, ao Velho, porque sei que não joga aos dados. 


\section{Resumo}

ARRIETA, E. A. Álgebras algébricas absolutamente valuadas. 2012. 120 f. Dissertação (Mestrado) - Instituto de Matemática e Estatística, Universidade de São Paulo, São Paulo, 2012.

O objetivo da dissertação é provar que toda álgebra, sobre o corpo dos números reais, algébrica e absolutamente valuada é de dimensão finita, e portanto isótopa a $\mathbb{R}, \mathbb{C}, \mathbb{H}$ ou $\mathbb{D}$. Observamos que $\mathbb{H}$ é a álgebra real dos Quatérnios e $\mathbb{D}$ a álgebra real dos Octônios. A demonstração do resultado é feita gradualmente, considerando inicialmente álgebras reais absolutamente valuadas algébrica com unidade, a seguir com unidade e finalmente, algébrica. Na demonstração do teorema será necessário combinar resultados não triviais de álgebras não associativas, análise funcional, álgebras de Banach e técnicas de ultraprodutos de espaços normados.

As álgebra absolutamente valuadas não são necessariamente associativas. Abraham Adrian Albert em 1947 mostrou que $\mathbb{R}, \mathbb{C}, \mathbb{H}$ e $\mathbb{D}$ são as únicas álgebras reais absolutamente valuadas de dimensão finita e com unidade; o mesmo Albert dois anos depois, em 1949, caracterizou essas mesmas álgebras como as únicas que são absolutamente valuadas algébricas e com unidade sobre os reais. Em 1960 Fred B. Wright e Kazimierz Urbanik provaram que $\mathbb{R}, \mathbb{C}$, $\mathbb{H}$ e $\mathbb{D}$ são as únicas álgebra reais absolutamente valuadas e com unidade. Recentemente, em 1997, Kaidi El-Amin, Maria Isabel Ramírez e Ángel Rodríguez Palacios mostraram que toda álgebra real absolutamente valuadas e algébrica é isótopa a uma de estas quatro. Nosso objetivo é desenvolver e unificar os resultados obtidos nestes 4 trabalhos.

Palavras-chave: Absolutamente valuada, Algébrica, Isótopa, Álgebras de Banach, Ultraprodutos. 


\section{Abstract}

ARRIETA, E. A. Absolute Valued Algebraic Algebras. 2012. 120 f. Dissertação (Mestrado) - Instituto de Matemática e Estatística, Universidade de São Paulo, São Paulo, 2012. Our goal here is to study the absolute valued algebraic real algebras. In order to reach our intention, we regard an absolute valued real algebra and on which one we impose: First, such one is finite-dimensional algebra; second; such one is algebraic algebra; third, such one is with unity; and in the end such one is algebraic algebra.

In the latter case, our aim, it needs of certain classic results of functional analysis and others one of Banach algebras; then, we reach that such one real algebra is isotope to one of the classical absolute valued real algebras $\mathbb{R}, \mathbb{C}, \mathbb{H}$ or $\mathbb{D}$. Where $\mathbb{H}$ is the Quaternions real algebra and $\mathbb{D}$ is the Octonions real algebra.

The absolute valued algebras are not necessarily associative. Abraham Adrian Albert was the first mathematician considering absolute valued algebras in a context not necessarily associative. In 1947, he proved that any finite-dimensional absolute valued real algebra with unit element is isomorphic to either real field $\mathbb{R}$, the complex field $\mathbb{C}$, the Quaternions algebra $\mathbb{H}$ or the Octonions algebra $\mathbb{D}$. Two years later, he demonstrated that $\mathbb{R}, \mathbb{C}, \mathbb{H}$ and $\mathbb{D}$ are the unique absolute valued algebraic real algebras with unit element.

Recently, in 1997, Kaidi El-Amin, Maria Isabel Ramírez and Ángel Rodríguez Palacios proved that any absolute valued algebraic real algebra is finite-dimensional.

Keywords: Absolute valued, Algebraic, isotope, Banach algebras. 


\section{Sumário}

Lista de Abreviaturas $\quad$ xi

Introdução $\quad$ xiii

1 Álgebras Absolutamente Valuadas $\quad 1$

1.1 Álgebras de Composição . . . . . . . . . . . . . . . . . 5 5

1.2 A.a.v. de Dimensão Finita com Unidade . . . . . . . . . . . . . . 12

1.3 A.a.v. Algébricas com Unidade . . . . . . . . . . . . . . 19

1.4 A.a.v. com Unidade . . . . . . . . . . . . . . . . . . 25

2 Espaços Normados e Álgebras de Banach 29

2.1 Espaços Normados . . . . . . . . . . . . . . . . . 29

2.2 Álgebra de Banach . . . . . . . . . . . . . . . . . . . . 35

2.3 Diferenciabilidade da Norma . . . . . . . . . . . . . . . 41

3 Âlgebras Algébricas Absolutamente Valuadas $\quad 51$

3.1 Ultrafiltros e Ultraprodutos . . . . . . . . . . . . . . . . . . 51

3.2 O Resultado . . . . . . . . . . . . . . . . . . 58

$\begin{array}{ll}\text { Referências Bibliográficas } & 61\end{array}$

$\begin{array}{ll}\text { Índice Remissivo } & 63\end{array}$ 


\section{Lista de Abreviaturas}

\begin{tabular}{|c|c|}
\hline$A$ & Álgebra sobre $\mathbb{F}$ \\
\hline A.a.v & Álgebra absolutamente valuada \\
\hline$A(a)$ & Subálgebra de $A$ gerada por $a$ \\
\hline $\mathbb{F}$ & Corpo \\
\hline$X, Y$ & Espaços métricos ou normados \\
\hline $\mathbb{H}$ & $\mathbb{R}$-álgebra dos Quatérnios \\
\hline $\mathbb{D}$ & $\mathbb{R}$-álgebra dos Octônios \\
\hline$R_{a}$ & Operador multiplicação à direita por $a$ \\
\hline$L_{a}$ & Operador multiplicação à esquerda por $a$ \\
\hline$\langle\rangle$. & Produto interno sobre um espaço vetorial \\
\hline$()$, & O associador de $A$ \\
\hline$\hat{X}$ & O completamento de $X$ \\
\hline$B L(X, Y)$ & O espaço das transformações lineares contínuas de $X$ em $Y$ \\
\hline$X^{\prime}$ & O espaço $B L(X, \mathbb{F})$ \\
\hline$\rho(x)$ & Resolvente de $x$ \\
\hline$\sigma(x)$ & O espectro de $x$ \\
\hline DTZ & Divisor topológico de zero \\
\hline $\operatorname{Inv}(A)$ & Conjunto dos elemento regulares numa álgebra de Banach \\
\hline $\operatorname{sing}(A)$ & Conjunto dos elementos singulares numa álgebra de Banach \\
\hline$\partial \operatorname{sing}(A)$ & A fronteira de $\operatorname{sing}(A)$ \\
\hline$f^{\prime}(x)$ & Derivada de Fréchet em $x$ \\
\hline $\operatorname{deg}(\mathrm{A})$ & Grau de uma álgebra de grau limitado \\
\hline $\mathcal{F}$ & Filtro sobre $I$ \\
\hline $\mathbb{F}\langle X\rangle$ & Álgebra livre não associativa sobre um conjunto não vazio $X$ \\
\hline $\mathcal{U}$ & Ultrafiltro sobre $I$ \\
\hline$\left(A_{i}\right)_{\mathcal{U}}$ & Ultraproduto de uma família de álgebras $A_{i}$ \\
\hline$\check{A}$ & Subálgebra de $A_{\mathcal{U}}$ isomorfa a $A$ \\
\hline $\lim _{\mathcal{U}}$ & Limite através do ultrafiltro $\mathcal{U}$ \\
\hline
\end{tabular}




\section{Introdução}

As álgebras reais absolutamente valuadas de dimensão finita surgem de forma natural ao considerar as soluções da seguinte equação quadrática

$$
\left(\sum_{i=1}^{n} x_{i}^{2}\right)\left(\sum_{i=1}^{n} y_{i}^{2}\right)=\sum_{i=1}^{n} z_{i}^{2}
$$

onde $x_{1}, \ldots, x_{n}, y_{1}, \ldots, y_{n}$ são indeterminadas independentes e $z_{i}=\sum_{j, k=1}^{n} a_{i j k} x_{j} y_{k}$ com $a_{i j k}$ números reais.

Adolf Hurwitz (1859 - 1919) mostrou em 1898 que se existir uma solução para dita equação quadrática, então $n=1,2,4$ ou 8. O teorema mostrado por A. Hurwitz estabelece que toda álgebra com unidade sobre o corpo dos números reais de dimensão finita e absolutamente valuada, com norma induzida por um produto interno, é isomorfa a $\mathbb{R}, \mathbb{C}, \mathbb{H}$ ou $\mathbb{D}$. Portanto, sua dimensão é 1, 2, 4 ou 8. Observamos que, $\mathbb{H}$ é a álgebra real dos Quatérnios e $\mathbb{D}$ a álgebra real dos Octônios.

A partir desse teorema de A. Hurwitz, temos duas linhas de pesquisa bem definidas:

i) A álgebra é considerada sobre qualquer corpo de característica diferente de dois e a dimensão não é necessariamente finita. Nesta direção, N. Jacobson, em [15], mostrou uma generalização do teorema de A. Hurwitz através das álgebras de composição. Tal prova é a matéria na Seção 1.1 do Capítulo 1. Vale a pena dizer aqui que em um trabalho de Irving Kaplansky, [17], ele mostrou o mesmo resultado, e nesta dissertação consideramos algumas das ideias da prova dada por Kaplansky para desenvolver a demonstração feita por N. Jacobson.

ii) Na outra direção, a álgebra é absolutamente valuada sobre o corpo dos números reais e a norma não é necessariamente induzida por um produto interno. Foi A.A. Albert, em [1], o primeiro em caracterizar as álgebras reais absolutamente valuada e de dimensão finita. O Teorema 1.6 apresenta tal caracterização. Albert, em [2], mostrou que o mesmo resultado é verdadeiro se a álgebra é algébrica. O Teorema 1.8 contém dita caracterização. É esta segunda linha a direção nesta dissertação.

Outro autor que obteve avanços importantes nesta última direção é Freb. B. Wright, [31], quem mostrou que toda álgebra real absolutamente valuada com unidade e de divisão é isomorfa com $\mathbb{R}, \mathbb{C}, \mathbb{H}$ ou $\mathbb{D}$. O mesmo F. B. Wright junto com Urbanik Kazimierz em [30], 
mostraram que os resultados obtidos por Albert são verdadeiros somente com as hipóteses que a álgebra seja absolutamente valuada com unidade. O Teorema 1.9 corresponde à prova dada por eles e em seguida damos um exemplo, segundo [30], que mostra a existência de álgebras absolutamente valuadas de dimensão infinita e assim encerramos o Capítulo 1.

Em [9], os autores K. El-Amin, M.I. Ramírez e A.R. Palacios, mostram que se uma álgebra real absolutamente valuada é algébrica, então é de dimensão finita. Nesta dissertação é apresentado, nos Capítulos 2 e 3, a matéria desenvolvida nesse trabalho mas a ordem dos assuntos é bem diferente. Na verdade os dois últimos capítulos apresentam uma bonita combinação entre alguns tópicos da análise funcional, álgebras de Banach e a teoria de ultraprodutos de espaços normados. Entre os resultados do Capítulo 2 destacamos o Corolário 2.1 que estabelece uma condição suficiente para que uma álgebra real absolutamente valuada seja de dimensão finita. É importante também o Corolário 2.4 pois, é utilizado de forma direta na prova do Teorema 3.2. De igual forma é importante neste capítulo as proposições 2.3, 2.4 e 2.5; já que são utilizadas de forma direta na prova de nosso objetivo, Teorema 3.2. O Capítulo 3 corresponde à matéria sobre ultraprodutos sendo o resultado principal o Teorema 3.1, também é importante neste capítulo o Corolário 3.4 o qual da a existência de um elemento de norma um em uma álgebra real algébrica absolutamente valuada, sobre o qual a norma é Fréchet diferenciável. 


\section{Capítulo 1}

\section{Álgebras Absolutamente Valuadas}

O objetivo desta dissertação é estudar as álgebras algébricas absolutamente valuadas sobre o corpo dos números reais. Uma álgebra $A$, diferente de zero, é dita álgebra absolutamente valuada, que nós abreviamos por a.a.v., se existir uma norma $\|$.$\| sobre A$ tal que $\|x y\|=\|x\|\|y\|$ para todo $x, y \in A$, e uma álgebra $A$ chama-se algébrica se para cada $x$ em $A$, a subálgebra gerada por $x$, denotada por $A(x)$, é de dimensão finita. Se a norma $\|$.$\| em A$ satisfaz $\|x y\| \leq\|x\|\|y\|$, para todo $x, y \in A$, então $A$ chama-se álgebra normada. Obviamente, as álgebras absolutamente valuadas são normadas.

As álgebras reais absolutamente valuadas de dimensão finita surgem de forma natural no famoso problema de Adolf Hurwitz. Consideremos a seguinte equação quadrática

$$
\left(\sum_{i=1}^{n} x_{i}^{2}\right)\left(\sum_{i=1}^{n} y_{i}^{2}\right)=\sum_{i=1}^{n} z_{i}^{2}
$$

onde $x_{1}, \ldots, x_{n}, y_{1}, \ldots, y_{n}$ são indeterminadas independentes e

$$
z_{i}=\sum_{j, k=1}^{n} a_{i j k} x_{j} y_{k}
$$

com $a_{i j k}$ números reais, isto implica que $z_{i}=z_{i}(X, Y)$ é uma forma bilinear em $X=$ $\left(x_{1}, \ldots, x_{n}\right)$ e $Y=\left(y_{1}, \ldots, y_{n}\right)$. A. Hurwitz mostrou que existem somente soluções não-triviais, nos termos $a_{i j k}$, para a equação acima, quando $n=1,2,4$ ou 8 .

Observamos que se $\left\{a_{i j k}\right\}_{1 \leq i, j, k \leq n}$ é uma solução para (1.1), então podemos definir uma álgebra $A$, sobre o corpo dos reais, de dimensão $n$, com produto $e_{i} e_{j}=\sum_{k=1}^{n} a_{i j k} e_{k}$, onde $\left\{e_{1}, \ldots, e_{n}\right\}$ é uma base de $A$ como $\mathbb{R}$-espaço vetorial. Se consideramos \|.\|, a norma em $A$ induzida pelo único produto interno que faz a base $\left\{e_{1}, \ldots, e_{n}\right\}$ ortonormal, então a equação (1.1) implica que $\|x y\|=\|x\|\|y\|$ para todo $x, y \in A$, isto é, $A$ é uma $\mathbb{R}$-álgebra absolutamente valuada.

Reciprocamente, suponhamos $A$ uma álgebra real absolutamente valuada de dimensão finita, com norma definida através de um produto interno. Seja $\left\{e_{1}, \ldots, e_{n}\right\}$ uma base ortonormal de $A$ e $\left\{a_{i j k}\right\}_{1 \leq i, j, k \leq n}$ as constantes de estrutura de $A$ em relação a esta base, isto é, $e_{i} e_{j}=\sum_{k=1}^{n} a_{i j k} e_{k}$. Se $x=\sum_{i=1}^{n} x_{i} e_{i}$ e $y=\sum_{j=1}^{n} y_{j} e_{j}$ são dois elementos arbitrários em $A$, então como $A$ é uma álgebra absolutamente valuada, segue que $\|x y\|^{2}=\|x\|^{2}\|y\|^{2}$, isto é,

$$
\left\|\sum_{i, j, k=1}^{n} a_{i j k} x_{i} y_{j} e_{k}\right\|^{2}=\left\|\sum_{i=1}^{n} x_{i} e_{i}\right\|^{2}\left\|\sum_{j=1}^{n} y_{j} e_{j}\right\|^{2} .
$$


Portanto,

$$
\sum_{k=1}^{n}\left(\sum_{i, j=1}^{n} a_{i j k} x_{i} y_{j}\right)^{2}=\left(\sum_{i=1}^{n} x_{i}{ }^{2}\right)\left(\sum_{j=1}^{n} y_{j}{ }^{2}\right) .
$$

Assim, $\left\{a_{i j k}\right\}_{1 \leq i, j, k \leq n}$ é uma solução da equação (1.1).

Consequentemente, a equação (1.1) possui soluções não triviais para um inteiro positivo $n$ se, e somente se, existir uma álgebra real absolutamente valuada de dimensão $n$, com norma induzida por um produto interno.

Vamos ilustrar, com alguns exemplos, como esta correspondência nos permite determinar soluções para a equação (1.1) de maneira natural.

Caso $n=1$. $\mathbb{R}$ é uma $\mathbb{R}$ - álgebra absolutamente valuada com relação à norma induzida pelo produto interno $\langle a, b\rangle=a b$, e $\{1\}$ é uma base ortonormal. Assim, $a_{111}=1$ e, portanto, uma solução de (1.1) para $n=1$ é dada por

$$
x^{2} y^{2}=(x y)^{2} .
$$

Caso $n=2$. Seja $A=\mathbb{C}$ a $\mathbb{R}$-álgebra dos números complexos. $\mathbb{C}$ é uma $\mathbb{R}$-álgebra absolutamente valuada com norma induzida pelo produto interno usual dos complexos, onde $\{1, i\}$ é uma base ortonormal. As constantes de estrutura desta base são

$$
a_{111}=1, a_{112}=0, a_{121}=0, a_{122}=1, a_{211}=0, a_{212}=1, a_{221}=-1, a_{222}=0
$$

que determinam a seguinte solução para (1.1),

$$
\left(x_{1}^{2}+x_{2}^{2}\right)\left(y_{1}^{2}+y_{2}^{2}\right)=\left(x_{1} y_{1}-x_{2} y_{2}\right)^{2}+\left(x_{2} y_{1}+x_{1} y_{2}\right)^{2} .
$$

Caso $n=4$. Seja $A=\mathbb{H}$, a $\mathbb{R}$-álgebra dos Quatérnios. $\mathbb{H}$ é uma álgebra de dimensão quatro e possui uma base, $\Phi=\{1, i, j, k\}$, com tabela de multiplicação dada por

\begin{tabular}{|c||c|c||c|c|}
\hline$\cdot$ & 1 & $i$ & $j$ & $k$ \\
\hline \hline 1 & 1 & $i$ & $j$ & $k$ \\
\hline$i$ & $i$ & -1 & $k$ & $-j$ \\
\hline \hline$j$ & $j$ & $-k$ & -1 & $i$ \\
\hline$k$ & $k$ & $j$ & $-i$ & -1 \\
\hline
\end{tabular}

Tabela 1.1: Tabela do produto dos Quatérnios

Se $x=x_{1} 1+x_{2} i+x_{3} j+x_{4} k$ e $y=y_{1} 1+y_{2} i+y_{3} j+y_{4} k$ são dois elementos arbitrários em $\mathbb{H}$, então definimos seu produto interno por $\langle x, y\rangle=\sum_{i=1}^{4} x_{i} y_{i}$. Em relação a este produto escalar, a base $\Phi$ é ortonormal. Se $\bar{x}=x_{1} 1-x_{2} i-x_{3} j-x_{4} k$, é o conjugado de $x$, obtemos que

$$
\bar{x} \bar{y}=\overline{y x} \quad \text { e } \quad \overline{\bar{x}}=x,
$$

para cada $x, y \in \mathbb{H}$. Para a norma induzida pelo produto escalar, dada por

$$
\|x\|=\sqrt{\langle x, x\rangle}=\sqrt{x \bar{x}}
$$

segue que $\|x y\|^{2}=(x y)(\overline{x y})=x(y \bar{y}) \bar{x}=(x \bar{x})(y \bar{y})=\|x\|^{2}\|y\|^{2}$. Assim, $\mathbb{H}$ é uma $\mathbb{R}$-álgebra absolutamente valuada e, portanto, as constantes de estrutura $\left\{a_{i j k}\right\}_{1<i, j, k<4}$, para a álgebra $\mathbb{H}$ em relação à base $\Phi$, determinam uma solução para a equação (1.1) dada por 


$$
\left(x_{1}^{2}+x_{2}^{2}+x_{3}^{2}+x_{4}^{2}\right)\left(y_{1}^{2}+y_{2}^{2}+y_{3}^{2}+y_{4}^{2}\right)=z_{1}^{2}+z_{2}^{2}+z_{3}^{2}+z_{4}^{2},
$$

com

$$
\begin{aligned}
& z_{1}=x_{1} y_{1}-x_{2} y_{2}-x_{3} y_{3}-x_{4} y_{4}, \\
& z_{2}=x_{2} y_{1}+x_{1} y_{2}-x_{4} y_{3}+x_{3} y_{4}, \\
& z_{3}=x_{3} y_{1}+x_{4} y_{2}+x_{1} y_{3}-x_{2} y_{4}, \\
& z_{4}=x_{4} y_{1}-x_{3} y_{2}+x_{2} y_{3}+x_{1} y_{4} .
\end{aligned}
$$

Esta solução foi descoberta por Euler no século XVIII, esquecida, e redescoberta por Hamilton no século XIX, ver [29]. Ao pouco tempo da redescoberta de Hamilton, Cayley descobriu uma identidade similar para 8-quadrados, que mostramos a seguir.

Caso $n=8$. Seja $A=\mathbb{D}$ a $\mathbb{R}$-álgebra dos Octônios. Temos que $\mathbb{D}$ é uma álgebra de dimensão oito e possui uma base, que denotaremos por $\Phi=\left\{1, i, j, k, i_{1}, i_{2}, i_{3}, i_{4}\right\}$, com tabela do produto dada por

\begin{tabular}{|c||c|c|c|c||c|c|c|c|}
\hline$\cdot$ & 1 & $i$ & $j$ & $k$ & $i_{1}$ & $i_{2}$ & $i_{3}$ & $i_{4}$ \\
\hline \hline 1 & 1 & $i$ & $j$ & $k$ & $i_{1}$ & $i_{2}$ & $i_{3}$ & $i_{4}$ \\
\hline$i$ & $i$ & -1 & $k$ & $-j$ & $i_{2}$ & $-i_{1}$ & $-i_{4}$ & $i_{3}$ \\
\hline$j$ & $j$ & $-k$ & -1 & $i$ & $i_{3}$ & $i_{4}$ & $-i_{1}$ & $-i_{2}$ \\
\hline$k$ & $k$ & $j$ & $-i$ & -1 & $i_{4}$ & $-i_{3}$ & $i_{2}$ & $-i_{1}$ \\
\hline \hline$i_{1}$ & $i_{1}$ & $-i_{2}$ & $-i_{3}$ & $-i_{4}$ & -1 & $i$ & $j$ & $k$ \\
\hline$i_{2}$ & $i_{2}$ & $i_{1}$ & $-i_{4}$ & $i_{3}$ & $-i$ & -1 & $-k$ & $j$ \\
\hline$i_{3}$ & $i_{3}$ & $i_{4}$ & $i_{1}$ & $-i_{2}$ & $-j$ & $k$ & -1 & $-i$ \\
\hline$i_{4}$ & $i_{4}$ & $-i_{3}$ & $i_{2}$ & $i_{1}$ & $-k$ & $-j$ & $i$ & -1 \\
\hline
\end{tabular}

Tabela 1.2: Tabela do produto dos Octônios

A base $\Phi$ é ortonormal com respeito ao produto interno definido por

$$
\langle x, y\rangle=\sum_{i=1}^{8} x_{i} y_{i}
$$

para $x=x_{1} 1+x_{2} i+x_{3} j+x_{4} k+x_{5} i_{1}+x_{6} i_{2}+x_{7} i_{3}+x_{8} i_{4}$ e $y=y_{1} 1+y_{2} i+y_{3} j+y_{4} k+y_{5} i_{1}+$ $y_{6} i_{2}+y_{7} i_{3}+y_{8} i_{4}$ elementos arbitrários da álgebra $\mathbb{D}$. A norma induzida por este produto escalar é dada por $\|x\|=\sqrt{\langle x, x\rangle}$, para cada elemento $x$ em $\mathbb{D}$.

Se $x=x_{1} 1+x_{2} i+x_{3} j+x_{4} k+x_{5} i_{1}+x_{6} i_{2}+x_{7} i_{3}+x_{8} i_{4}$ em $\mathbb{D}$, então o conjugado de $x$ é definido por $\bar{x}=x_{1} 1-x_{2} i-x_{3} j-x_{4} k-x_{5} i_{1}-x_{6} i_{2}-x_{7} i_{3}-x_{8} i_{4}$. É imediato mostrar que a aplicação $-: \mathbb{D} \longrightarrow \mathbb{D}$, dada por $x \mapsto \bar{x}$ satisfaz as seguintes propriedades:

$$
\overline{(x+y)}=\bar{x}+\bar{y}, \quad \overline{1}=1, \quad \overline{\bar{x}}=x,
$$

para todo $x, y$ em $\mathbb{D}$.

A álgebra real dos Octônios pode ser obtida da álgebra real associativa dos Quatérnios através do processo de duplicação de Cayley-Dickson. Através deste processo podemos identificar cada elemento de $\mathbb{D}$ como um par de elementos de $\mathbb{H}$. Dado $x=x_{1} 1+x_{2} i+x_{3} j+$ $x_{4} k+x_{5} i_{1}+x_{6} i_{2}+x_{7} i_{3}+x_{8} i_{4} \in \mathbb{D}$, representamos $x$ como um par de quatérnios $x=(a, b)$ com $a=x_{1} 1+x_{2} i+x_{3} j+x_{4} k$ e $b=x_{5} 1+x_{6} i+x_{7} j+x_{8} k$ em $\mathbb{H}$. Assim, a base $\Phi$ de $\mathbb{D}$, é 
identificada com os pares

$$
\{(1,0),(i, 0),(j, 0),(k, 0),(0,1),(0, i),(0, j),(0, k)\} .
$$

A soma e o produto dos pares é dada por:

$$
\begin{gathered}
(a, b)+(c, d)=(a+c, b+d), \\
(a, b) \cdot(c, d)=(a c-\bar{d} b, d a+b \bar{c}) .
\end{gathered}
$$

Com esta identificação, o conjugado de $x=(a, b)$ é, $\overline{(a, b)}=(\bar{a},-b)$, onde $\bar{a}$ é o conjugado de $a$ em $\mathbb{H}$. Daí, é fácil mostrar que

$$
\overline{x y}=\bar{y} \bar{x},
$$

para todo $x, y$ em $\mathbb{D}$. De fato, se $y=(c, d)$, então $x y=(a c-\bar{d} b, d a+b \bar{c})$, portanto $\overline{x y}=(\bar{c} \bar{a}-\bar{b} d,-d a-b \bar{c})=(\bar{c},-d)(\bar{a},-b)=\bar{y} \bar{x}$. Finalmente,

$$
\begin{aligned}
\|x y\|^{2} & =\|(x y)(\overline{x y})\|=\|(a c-\bar{d} b, d a+b \bar{c})(\bar{c} \bar{a}-\bar{b} d,-d a-b \bar{c})\| \\
& =\left\|\left(\|a\|^{2}\|c\|^{2}-a c \bar{b} d-\bar{d} b \bar{c} \bar{a}+\|b\|\left\|^{2}\right\| d\left\|^{2}+\right\| a\left\|^{2}\right\| d\left\|^{2}+\bar{a} \bar{u}+u a+\right\| b\left\|^{2}\right\| c \|^{2}, 0\right)\right\| \\
& =\left\|\left(\left(\|a\|^{2}+\|b\|^{2}\right)\left(\|c\|^{2}\|d\|^{2}\right)-a u-\overline{a u}+u a+\overline{u a}, 0\right)\right\| \\
& =\left\|\left(\left(\|a\|^{2}+\|b\|^{2}\right)\left(\|c\|^{2}+\|d\|^{2}\right), 0\right)\right\|=\left(\|a\|^{2}+\|b\|^{2}\right)\left(\|c\|^{2}+\|d\| \|^{2}\right)=\|x\|^{2}\|y\|^{2}
\end{aligned}
$$

onde $u=c \bar{b} d$. Portanto, $\mathbb{D}$ é uma álgebra real absolutamente valuada. Assim, as constantes de estrutura da álgebra, com respeito à base ortonormal $\Phi$, determinam uma solução para a equação (1.1) dada por

$$
\left(\sum_{i=1}^{8} x_{i}^{2}\right)\left(\sum_{i=1}^{8} y_{i}^{2}\right)=\sum_{i=1}^{8} z_{i}^{2}
$$

onde

$$
\begin{aligned}
& z_{1}=x_{1} y_{1}-x_{2} y_{2}-x_{3} y_{3}-x_{4} y_{4}-x_{5} y_{5}-x_{6} y_{6}-x_{7} y_{7}-x_{8} y_{8}, \\
& z_{2}=x_{1} y_{2}+x_{2} y_{1}+x_{3} y_{4}-x_{4} y_{3}+x_{5} y_{6}-x_{6} y_{5}-x_{7} y_{8}+x_{8} y_{7}, \\
& z_{3}=x_{1} y_{3}-x_{2} y_{4}+x_{3} y_{1}+x_{4} y_{2}+x_{5} y_{7}+x_{6} y_{8}-x_{7} y_{5}-x_{8} y_{6}, \\
& z_{4}=x_{1} y_{4}+x_{2} y_{3}-x_{3} y_{2}+x_{4} y_{1}+x_{5} y_{8}-x_{6} y_{7}+x_{7} y_{6}-x_{8} y_{5}, \\
& z_{5}=x_{1} y_{5}-x_{2} y_{6}-x_{3} y_{7}-x_{4} y_{8}+x_{5} y_{1}+x_{6} y_{2}+x_{7} y_{3}+x_{8} y_{4}, \\
& z_{6}=x_{1} y_{6}+x_{2} y_{5}-x_{3} y_{8}+x_{4} y_{7}-x_{5} y_{2}+x_{6} y_{1}-x_{7} y_{4}+x_{8} y_{3}, \\
& z_{7}=x_{1} y_{7}+x_{2} y_{8}+x_{3} y_{5}-x_{4} y_{6}-x_{5} y_{3}+x_{6} y_{4}+x_{7} y_{1}-x_{8} y_{2}, \\
& z_{8}=x_{1} y_{8}-x_{2} y_{7}+x_{3} y_{6}+x_{4} y_{5}-x_{5} y_{4}-x_{6} y_{3}+x_{7} y_{2}+x_{8} y_{1} .
\end{aligned}
$$

O famoso resultado que A. Hurwitz mostrou estabelece o seguinte :

Teorema 1.1 Seja $\mathbb{F}$ um corpo de caracteristica diferente de 2. Se existir uma identidade

$$
\left(x_{1}^{2}+\cdots+x_{n}^{2}\right)\left(y_{1}^{2}+\cdots+y_{n}^{2}\right)=z_{1}^{2}+\cdots+z_{n}^{2},
$$

para todo $x_{1}, \ldots, x_{n}, y_{1}, \ldots, y_{n}$ em $\mathbb{F}$, onde cada $z_{k}$ é uma função $\mathbb{F}$-bilinear nas $x$ 's e y's, então $n=1,2,4$ ou 8.

A prova original de Hurwitz foi sobre o corpo $\mathbb{F}=\mathbb{C}$, porém a prova também é valida sobre qualquer corpo $\mathbb{F}$ de característica diferente de dois. Se a característica de $\mathbb{F}$ for dois então existe uma solução para a relação acima para todo $n$, já que em característica 2 a suma de quadrados também é um quadrado. 
Observamos que uma álgebra real absolutamente valuada de dimensão finita, induzida por uma solução da equação (1.1), não é necessariamente com unidade. Esta situação nos leva a introduzir uma bonita idéia de A.A. Albert, o conceito de álgebras isótopas, que nos permite relacionar as álgebras absolutamente valuadas sem unidade com as álgebras absolutamente valuadas com unidade. Seja $A$ uma $\mathbb{R}$-álgebra absolutamente valuada de dimensão finita (não necessariamente com unidade). Então, para cada $a \in A$ com $\|a\|=1$, os operadores em $A$ dados por $R_{a}: x \mapsto x a, L_{a}: x \mapsto a x$, multiplicação à direita e multiplicação à esquerda respectivamente, satisfazem $\left\|R_{a}(x)\right\|=\|x a\|=\|x\|=\|a x\|=\left\|L_{a}(x)\right\|$. Assim, $R_{a}$ e $L_{a}$ são aplicações lineares injetoras e, como $A$ é de dimensão finita, são bijetoras. Daí, $R_{a}$ e $L_{a}$ são operadores ortogonais e, portanto, $R_{a}^{-1}$ e $L_{a}^{-1}$ também são ortogonais. Fixado um $a \in A$ de norma um, definimos um novo produto, denotado por ".", em $A$ dado por

$$
x \cdot y=R_{a}^{-1}(x) L_{a}^{-1}(y)
$$

Obtemos assim, uma nova álgebra real absolutamente valuada com a mesma norma de $A$ e onde $e=a^{2}$ é unidade, visto que para cada $x \in A$, temos

$$
e \cdot x=R_{a}^{-1}\left(a^{2}\right) L_{a}^{-1}(x)=a L_{a}^{-1}(x)=x, \quad x \cdot e=R_{a}^{-1}(x) L_{a}^{-1}\left(a^{2}\right)=R_{a}^{-1}(x) a=x .
$$

A nova álgebra obtida a partir de $A$ e com produto o produto denotado por ".", chama-se álgebra isótopa de $A$ (segundo A.A. Albert em [1]).

Definição 1.1 Duas álgebras sobre o mesmo corpo, $A_{1}$ e $A_{2}$, são isótopas se são iguais como espaços vetoriais e o produto em $A_{2}$, denotado por $\cdot$, é obtido do produto de $A_{1}$, denotado por justaposição, através da seguinte fórmula

$$
x \cdot y=T_{1}(x) T(y),
$$

sendo $T$ e $T_{1}$ operadores não singulares sobre $A_{1}$.

Temos assim o seguinte fato importante:

Lema 1.1 Toda $\mathbb{R}$-álgebra absolutamente valuada e de dimensão finita é isótopa a uma álgebra real absolutamente valuada com unidade.

Em [8] encontramos que a idéia mostrada acima, pode-se generalizar para álgebras sobre um corpo arbitrário. Seja $A$ um álgebra sobre um corpo $\mathbb{F}$ tal que existam $a, b \in A$ onde $L_{a}$ e $R_{b}$ são bijetoras. Então $A$ é isótopa a uma álgebra com unidade, com produto definido por $x \cdot y=R_{b}^{-1}(x) L_{a}^{-1}(y)$, com unidade $e=a b$.

A seguir veremos dois métodos para provar o Teorema de A. Hurwitz. No primeiro, são utilizadas as álgebras de composição e o segundo através das álgebras absolutamente valuadas. Em [22] é dada uma outra prova do Teorema de A. Hurwitz usando técnicas de álgebras de Clifford.

\section{1 Álgebras de Composição}

Nesta seção é dada uma prova do teorema de A. Hurwitz através das álgebras de composição sobre um corpo $\mathbb{F}$ de característica diferente de dois. Primeiro mostramos que toda álgebra de composição é quadrática e alternativa, em seguida introduzimos o processo de duplicação de Cayley-Dickson e mostramos que se $A_{0}$ é uma subálgebra própria, de dimensão 
finita, de uma álgebra de composição $A$ tal que $1 \in A_{0}$ e a forma quadrática é não degenerada sobre $A_{0}$, então $A$ contém uma subálgebra isomorfa à duplicação de $A_{0}$. O assunto nesta seção segue as linhas de [15], [17], [20] e [27].

Observamos, que cada $\mathbb{R}$-álgebra absolutamente valuada de dimensão finita com norma induzida por um produto interno, pode ser considerada como uma álgebra de composição, portanto, toda $\mathbb{R}$-álgebra induzida por uma solução da equação (1.1) é isótopa a uma $\mathbb{R}$ álgebra de composição.

Vejamos agora como a teoria das álgebras de composição nos permite provar o Teorema de A. Hurwitz. Seja $A$ uma $\mathbb{F}$-álgebra, com característica de $\mathbb{F}$ diferente de dois, e $Q: A \longrightarrow \mathbb{F}$ uma forma quadrática não degenerada que admite composição, isto é,

$$
Q(x y)=Q(x) Q(y)
$$

para todo $x, y$ em $A$. Uma forma quadrática $Q$ é não degenerada se a forma bilinear simétrica associada

$$
Q(x, y):=Q(x+y)-Q(x)-Q(y)
$$

e não degenerada, no sentido que seu radical $\operatorname{rad}(Q):=\{z \in A: Q(z, x)=0\}$ é zero. Uma álgebra de composição é um par formado por uma álgebra (não necessariamente associativa) que possui unidade junto com uma forma quadrática não degenerada que admite composição. Observemos que a lei de composição dada pela Equação (1.2) implica que $Q(x) Q(1)=Q(x)$ para todo $x \in A$, $\log o$

$$
Q(1)=1
$$

O traço $T$ é definido por

$$
T(x):=Q(1, x) .
$$

Substituindo em (1.2) o fator $y$ por $y+z$, obtemos

$$
Q(x y, x z)=Q(x) Q(y, z) \quad(x, y, z \in A) .
$$

Se em (1.4) trocamos $x$ por $x+1$, obtemos

$$
Q(x y, z)+Q(y, x z)=T(x) Q(y, z) \quad(x, y, z \in A)
$$

Substituindo $y$ por $x y$ em $(1.5)$ temos $Q(x(x y), z)+Q(x y, x z)=T(x) Q(x y, z)$ que combinando com a relação (1.4) seque

$$
Q(x(x y), z)+Q(x) Q(y, z)=T(x) Q(x y, z) .
$$

Portanto, $Q(x(x y)-T(x) x y+Q(x) y, z)=0$ para todo $z \in A$. Como $Q$ é não degenerada, podemos afirmar que

$$
x(x y)-T(x) x y+Q(x) y=0 .
$$

Portanto quando $y=1$ obtemos que

$$
x^{2}-T(x) x+Q(x) 1=0,
$$

para todo elemento $x$ em $A$. Por outro lado, a subtração, da equação (1.7) multiplicada pela direita por $y$, e a equação (1.6), nos determina a relação $x^{2} y=x(x y)$ para todo $x, y \in A$. Por dualidade, seque que $y x^{2}=(y x) x$, para todo $x, y \in A$.

Uma $\mathbb{F}$-álgebra com unidade e característica de $\mathbb{F}$ diferente de dois, é dita quadrática se para todo $x$ na álgebra, o conjunto $\left\{1, x, x^{2}\right\}$ é linearmente dependente. Para cada $x, y, z \in A$, 
definimos o associador de $A,(x, y, z)$ por

$$
(x, y, z):=(x y) z-x(y z) .
$$

Uma álgebra é dita alternativa se para todo par de elementos $x, y$ na álgebra temos que $(x, x, y)=0$ e $(y, x, x)=0$. Observamos que uma álgebra é alternativa se, e somente se, cada subálgebra gerada por dois elementos é associativa.

Provamos acima os seguintes resultados.

Lema 1.2 Seja A uma $\mathbb{F}$-álgebra de composição sobre um corpo $\mathbb{F}$, de característica diferente de dois. Então A é alternativa, quadrática e satisfaz a identidade

$$
x^{2}-T(x) x+Q(x) 1=0,
$$

para todo elemento $x$ em $A$.

Lema 1.3 Em toda álgebra alternativa sobre um corpo $\mathbb{F}$ de característica diferente de 2, temos que o associador é uma aplicação trilinear alternada e satisfaz a identidade de Moufang, isto é,

$$
(z x)(y z)=z(x y) z, \quad(\text { Identidade de Moufang })
$$

para todo $x, y, z$ na álgebra.

\section{Demonstração:}

Para mostrar que o associador é uma aplicação alternada é suficiente provar que $(x, y, x)=0$ para todo $x, y$ na álgebra. Linearizando a identidade $(x, x, y)=0$ obtemos que $(x, z, y)=$ $-(z, x, y)$ e portanto $(x, y, x)=-(y, x, x)=0$.

Como o associador é uma aplicação alternada temos que, $(z, x, y)=(x, y, z)$ para todo $x, y, z$ na álgebra, isto é,

$$
(z x) y+x(y z)=z(x y)+(x y) z
$$

Se substituímos $x$ por $z x$ na equação (1.8) obtemos $\left(z^{2} x\right) y+(z x)(y z)=z((z x) y)+((z x) y) z$. Se agora substituímos $y$ por $y z$ na mesma equação (1.8) segue que, $(z x)(y z)+x\left(y z^{2}\right)=$ $z(x(y z))+(x(y z)) z$. Então, somando as igualdades que obtemos das substituições, segue:

$$
\begin{aligned}
\left(z^{2} x\right) y+2(z x)(y z)+x\left(y z^{2}\right) & =z((z x) y)+((z x) y) z+z(x(y z))+(x(y z)) z \\
& =z((z x) y+x(y z))+((z x) y+x(y z)) z \\
& =z(z(x y)+(x y) z)+(z(x y)+(x y) z) z \\
& =z^{2}(x y)+z(x y) z+z(x y) z+(x y) z^{2} \\
& =z^{2}(x y)+2 z(x y) z+(x y) z^{2}
\end{aligned}
$$

Substituindo $z$ por $z^{2}$ na equação (1.8) resulta que,

$$
\left(z^{2} x\right) y+x\left(y z^{2}\right)=z^{2}(x y)+(x y) z^{2} .
$$

Assim, $z^{2}(x y)+2(z x)(y z)+(x y) z^{2}=z^{2}(x y)+2 z(x y) z+(x y) z^{2}$ e como a característica de $\mathbb{F}$ é diferente de dois obtemos a identidade de Moufang.

A involução canônica de $A$ é uma aplicação linear definida por

$$
\bar{x}:=T(x) 1-x,
$$


para todo $x \in A$. Por equação (1.5) seque imediatamente que

$$
Q(x y, z)=Q(y, T(x) z-x z)=Q(y, \bar{x} z) .
$$

Por dualidade de (1.4) segue que $Q(y x, z, x)=Q(x) Q(y, z)$ e substituindo $x$ por $x+1$, obtemos de forma análoga, a relação dual de (1.9)

$$
Q(y x, z)=Q(y, z \bar{x}) .
$$

Lema 1.4 Em cada álgebra de composição A a involução canônica é uma involução, isto é,

$$
\overline{1}=1 \quad \overline{\bar{x}}=x \quad \overline{x y}=\bar{y} \bar{x}
$$

e satisfaz

$$
T(\bar{x})=T(x), \quad \bar{x} x=Q(x) 1=x \bar{x},
$$

para todo $x$ em $A$.

\section{Demonstração:}

Como $T(1)=Q(1,1)=2 Q(1)=2$, segue que $\overline{1}=T(1) 1-1=1$.

Vejamos que $\overline{\bar{x}}=x$. Pela definição de $T$, obtemos $T(\bar{x})=Q(\bar{x}, 1)=Q(T(x) 1-x, 1)=$ $T(x) Q(1,1)-Q(x, 1)=2 T(x)-T(x)=T(x)$. Então

$$
\overline{\bar{x}}=T(\bar{x}) 1-\bar{x}=T(x) 1-(T(x) 1-x)=x
$$

para todo $x \in A$. Usando as equações (1.9) e (1.10) obtemos que

$$
Q(z,(\overline{x y}-\bar{y} \bar{x}))=Q(z, \overline{x y})-Q(z, \bar{y} \bar{x})=Q(x y, \bar{z})-Q(x, \bar{z} \bar{y})=Q(x, \bar{z} \bar{y})-Q(x, \bar{z} \bar{y})=0,
$$

para todo $z \in A$. Pelo fato de que $Q$ é não degenerada segue que, $\overline{x y}=\bar{y} \bar{x}$.

Substituindo a $T(x)$ por $\bar{x}+x$ na equação (1.7) obtemos

$$
x^{2}-(\bar{x}+x) x+Q(x) 1=0=x^{2}-x(\bar{x}+x)+Q(x) 1,
$$

portanto, $\bar{x} x=Q(x) 1=x \bar{x}$, para todo $x \in A$.

A construção de Cayley-Dickson. Dada uma álgebra de composição de dimensão finita, podemos obter uma nova álgebra com dimensão duas vezes a dimensão da álgebra, através do processo de duplicação de Cayley-Dickson. Se $\operatorname{dim} A<\infty$, e $\alpha \in \mathbb{F} \backslash\{0\}$, no $\mathbb{F}$-espaço vetorial $A \oplus A$, a soma direta de duas copias de $A$, formada por pares ordenados $(x, y)$ para $x, y \in A$, definimos um novo produto e involução por

$$
\begin{gathered}
(a, b) \cdot(x, y)=(a x+\alpha \bar{y} b, y a+b \bar{x}), \\
(x, y)^{*}=(\bar{x},-y),
\end{gathered}
$$

para todo $a, b, x, y \in A$. A nova álgebra, denotada por $A(\alpha)$, tem unidade $\mathbf{1}=(1,0)$ e a aplicação linear " $*^{\prime \prime}$, é uma involução pois $\mathbf{1}^{*}=\mathbf{1},(x, y)^{* *}=(x, y) \mathrm{e}$

$$
((a, b) \cdot(x, y))^{*}=(\overline{a x+\alpha \bar{y} b},-y a-b \bar{x})=(\bar{x},-y) \cdot(\bar{a},-b)=(x, y)^{*} \cdot(a, b)^{*} .
$$

Podemos dizer que $A$ é uma subálgebra de $A(\alpha)$ através do monomorfismo de álgebras

$$
x \mapsto(x, 0)
$$


Assim identificamos cada elemento $x$ de $A$ com o par $(x, 0)$ em $A(\alpha)$ e se representamos $(0,1)$ por $\ell$, então podemos identificar o par $(0, y)=(y, 0) \cdot(0,1)$ com $y \ell \in A \ell$. Portanto, escrevemos $(x, y)=x+y \ell$. Segundo esta notação, $A(\alpha)=A \oplus A \ell$. O produto e a involução podem-se escrever como

$$
\begin{gathered}
(a+b \ell) \cdot(x+y \ell)=(a x+\alpha \bar{y} b)+(y a+b \bar{x}) \ell, \\
(x+y)^{*}=\bar{x}-y \ell .
\end{gathered}
$$

Portanto, $A$ é uma subálgebra de $A(\alpha)$ e temos as seguintes regras do produto de CayleyDickson:

$$
\begin{aligned}
x \ell & =\ell \bar{x}, \\
x(y \ell) & =(y x) \ell, \\
(x \ell) y & =(x \bar{y}) \ell, \\
(x \ell)(y \ell) & =\alpha \bar{y} x .
\end{aligned}
$$

A involução satisfaz as seguintes propriedades

$$
(x+y \ell) \cdot(x+y \ell)^{*}=(x+y \ell) \cdot(\bar{x}-y \ell)=(x \bar{x}-\alpha \bar{y} y)+(-y x+y \overline{\bar{x}}) \ell=(Q(x)-\alpha Q(y)) \mathbf{1}
$$

e

$$
(x+y \ell)+(x+y \ell)^{*}=(x+\bar{x})
$$

para todo $x, y \in A$.

Vamos a ilustrar com um exemplo, como funciona o processo de duplicação de CayleyDickson. Seja $A=\mathbb{F} 1$, o corpo $\mathbb{F}$. Por duplicação obtemos $A(\alpha)$, uma álgebra de dimensão dois, que possui uma base $\{1, i\}$ tal que 1 é a unidade da álgebra e $i^{2}=\alpha 1$. Obviamente $A(\alpha)$ é uma álgebra quadrática. Duplicando a álgebra quadrática $A(\alpha)$, obtemos uma $\mathbb{F}$ álgebra de dimensão quatro $A(\alpha, \beta)$, que chamamos álgebra generalizada dos Quatérnios. Se agora duplicamos a álgebra generalizada dos Quatérnios, obtemos $A(\alpha, \beta, \gamma)$, uma álgebra de dimensão oito sobre $\mathbb{F}$, chamada álgebra generalizada dos Octônios. Cada uma das $\mathbb{F}$-álgebras de composição obtidas acima a partir de $\mathbb{F} 1$ através do processo de duplicação de CayleyDickson são chamadas álgebras de Hurwitz sobre $\mathbb{F}$. A tabela do produto de $A(\alpha, \beta, \gamma)$ é dada por:

\begin{tabular}{|c||c|c|c|c||c|c|c|c|}
\hline$\cdot$ & 1 & $i$ & $j$ & $k$ & $\ell$ & $i \ell$ & $j \ell$ & $k \ell$ \\
\hline \hline 1 & 1 & $i$ & $j$ & $k$ & $\ell$ & $i \ell$ & $j \ell$ & $k \ell$ \\
\hline$i$ & $i$ & $\alpha 1$ & $k$ & $\alpha j$ & $i \ell$ & $\alpha \ell$ & $-k \ell$ & $-\alpha j \ell$ \\
\hline$j$ & $j$ & $-k$ & $\beta 1$ & $-\beta i$ & $j \ell$ & $k \ell$ & $\beta \ell$ & $\beta i \ell$ \\
\hline$k$ & $k$ & $-\alpha j$ & $\beta i$ & $-\alpha \beta 1$ & $k \ell$ & $\alpha j \ell$ & $-\beta i \ell$ & $-\alpha \beta \ell$ \\
\hline \hline$\ell$ & $\ell$ & $-i \ell$ & $-j \ell$ & $-k \ell$ & $\gamma 1$ & $-\gamma i$ & $-\gamma j$ & $-\gamma k$ \\
\hline$i \ell$ & $i \ell$ & $-\alpha \ell$ & $-k \ell$ & $-\alpha j \ell$ & $\gamma i$ & $-\alpha \gamma 1$ & $\gamma k$ & $\alpha \gamma j$ \\
\hline$j \ell$ & $j \ell$ & $k \ell$ & $-\beta \ell$ & $\beta i \ell$ & $\gamma j$ & $-\gamma k$ & $-\beta \gamma 1$ & $-\beta \gamma i$ \\
\hline$k \ell$ & $k \ell$ & $\alpha j \ell$ & $-\beta i \ell$ & $\alpha \beta \ell$ & $\gamma k$ & $-\alpha \gamma j$ & $\beta \gamma i$ & $\alpha \beta \gamma 1$ \\
\hline
\end{tabular}

Tabela 1.3: Tabela do produto das $\mathbb{F}$-álgebras de Hurwitz

Observemos que se as constantes de duplicação $\alpha, \beta$ e $\gamma$ são iguais a -1 e $\mathbb{F}=\mathbb{R}$ a Tabela 1.3 corresponde à Tabela 1.2 . 
Se agora duplicamos a álgebra generalizada dos Octônios, obtemos $A(\alpha, \beta, \gamma, \delta)$, uma álgebra de dimensão dezesseis sobre $\mathbb{F}$. Representemos por $\mathfrak{m}$ ao par $(0,1)$ em $A(\alpha, \beta, \gamma, \delta)$. Usando as regras do produto de Cayley-Dickson, (Equações (1.11), (1.12), (1.13) e (1.14)), obtemos o seguinte resultado.

Lema 1.5 A álgebra $A(\alpha, \beta, \gamma, \delta)$ não é alternativa, portanto, não é de composição.

\section{Demonstração:}

Temos que

$$
\begin{aligned}
(i, j, \ell \mathfrak{m}) & =(i j)(\ell \mathfrak{m})-i(j(\ell \mathfrak{m}))=k(\ell \mathfrak{m})-i((\ell j) \mathfrak{m}) \quad(\text { Por 1.12) } \\
& =(\ell k) \mathfrak{m}-((\ell j) i) \mathfrak{m}=(\bar{k} \ell) \mathfrak{m}-((\bar{j} \ell) i) \mathfrak{m} \quad(\text { Por 1.11) } \\
& =-(k \ell) \mathfrak{m}+((j \ell) i) \mathfrak{m}=-(k \ell) \mathfrak{m}+((j \bar{i}) \ell) \mathfrak{m}=-(k \ell) \mathfrak{m}+(k \ell) \mathfrak{m}=0 .
\end{aligned}
$$

Por outro lado,

$$
\begin{aligned}
(i, \ell \mathfrak{m}, j) & =(i(\ell \mathfrak{m})) j-i((\ell \mathfrak{m}) j)=((\ell i) \mathfrak{m}) j-i((\ell \bar{j}) \mathfrak{m}) \quad(\text { Por } 1.12 \text { e } 1.13) \\
& =((\ell i) \bar{j}) \mathfrak{m}-((\ell \bar{j}) i) \mathfrak{m}=((\bar{i} \ell) \bar{j}) \mathfrak{m}-((j \ell) i) \mathfrak{m} \quad(\text { Por } 1.11 \text { e } 1.13) \\
& =-((i \ell) \bar{j}) \mathfrak{m}-((j \ell) i) \mathfrak{m}=-((i j) \ell) \mathfrak{m}-((j \bar{i}) \ell) \mathfrak{m}=-(k \ell) \mathfrak{m}-((i j) \ell) \mathfrak{m} \\
& =-2(k \ell) \mathfrak{m} \neq 0,
\end{aligned}
$$

pois $\mathbb{F}$ é um corpo de característica diferente de dois. Assim o associador sobre a álgebra não é uma aplicação alternada. Portanto, $A(\alpha, \beta, \gamma, \delta)$ não é alternativa.

Na verdade pode-se provar de maneira direta, usando as definições, o seguinte lema.

Lema 1.6 Seja A uma $\mathbb{F}$-álgebra com 1 e com involução escalar, isto é, $x \bar{x}$ e $x+\bar{x}$ são elementos de $\mathbb{F} 1$ para todo $x \in A$, e $x \bar{x}=Q(x) 1$ onde $Q$ é uma forma quadrática não degenerada sobre A. Então:

i) $A(\alpha)$ sempre tem uma involução escalar não trivial;

ii) $A(\alpha)$ é comutativa e associativa se, e somente se, A é comutativa, associativa e a involução é a identidade;

iii) $A(\alpha)$ é associativa se, e somente se, $A$ é comutativa e associativa;

iv) $A(\alpha)$ é alternativa se, e somente se, $A$ é associativa.

Também precisamos do seguinte fato importante válido em $A$.

Lema 1.7 Sejam A uma álgebra de composição sobre um corpo $\mathbb{F}$ de característica diferente de 2, $A_{0}$ uma subálgebra própria de dimensão finita que contém o 1 e a forma quadrática sobre $A_{0}$ é não degenerada. Então existem elementos $\ell \in A_{0}^{\perp} \operatorname{com} Q(\ell)=-\alpha \neq 0$ e para um tal elemento $A_{0}+A_{0} \ell$ é uma subálgebra de $A$ isomorfa a $A_{0}(\alpha)$.

\section{Demonstração:}

Como a forma quadrática $Q$ é não degenerada sobre $A_{0}$ e dado que $\operatorname{dim}\left(A_{0}\right)<\infty$, podemos escrever $A=A_{0} \oplus A_{0}^{\perp}$. Também temos que $A_{0}^{\perp} \neq 0$, pois $A_{0}$ é uma subálgebra própria de $A$. Como $Q$ é não degenerada segue que $0 \neq Q\left(A_{0}^{\perp}, A\right)=Q\left(A_{0}^{\perp}, A_{0} \oplus A_{0}^{\perp}\right)=Q\left(A_{0}^{\perp}, A_{0}^{\perp}\right)$. 
Portanto, $Q$ não é identicamente zero sobre $A_{0}^{\perp}$. Daí, existe $\ell \neq 0$ em $A_{0}^{\perp}$ tal que $Q(\ell)=$ $-\alpha \neq 0$. Seja $x \ell \in A_{0} \ell$. Pela equação (1.9), segue que

$$
Q(x \ell, y)=Q(\ell, \bar{x} y)=0
$$

para todo $y \in A_{0}$. Assim, $A_{0} \ell \subset A_{0}^{\perp}$ e portanto,

$$
K=A_{0} \oplus A_{0} \ell
$$

Dado $x+y \ell$ em $K$, segue que

$$
\begin{aligned}
Q(x+y \ell) & =Q(x)+Q(y \ell)+Q(x, y \ell)=Q(x)+Q(y) Q(\ell)=Q(x)-\alpha Q(y), \\
T(x+y \ell) & =Q(x+y \ell, 1)=Q(x, 1)=T(x), \\
\overline{x+y \ell} & =T(x+y \ell) 1-(x+y \ell)=\bar{x}-y \ell .
\end{aligned}
$$

Mostraremos que $K$ é uma subálgebra de $A$ que satisfaz as regras do produto de CayleyDickson. (Equações (1.11), (1.12), (1.13) e (1.14)). Primeiro observamos que o escalar $\alpha$ representa o quadrado do elemento $\ell$,

$$
\ell^{2}=\alpha 1
$$

já que por relação $\bar{\ell}=-\ell$, e Lema 1.4 seque que $\ell^{2}=-\bar{\ell} \ell=-Q(\ell) 1=-(-\alpha) 1=\alpha 1$.

Por outro lado, se $x \in A_{0}$, temos que $x \ell=-\overline{x \ell}=-\bar{\ell} \bar{x}=\ell \bar{x}$, isto é

$$
x \ell=\ell \bar{x} .
$$

Agora, usando que $A$ é alternativa, a relação acima e a identidade de Moufang, obtemos

$$
(x \ell)(y \ell)=(\ell \bar{x})(y \ell)=\ell(\bar{x} y) \ell=\ell((\bar{x} y) \ell)=\ell(\ell(\bar{y} x))=\ell^{2}(\bar{y} x)=\alpha \bar{y} x,
$$

para todo $x, y$ em $A_{0}$. Agora, temos que

$$
\begin{array}{rlr}
0 & =(\ell, \bar{x}, \bar{y})+(\bar{x}, \ell, \bar{y})=(\ell \bar{x}) \bar{y}-\ell(\bar{x} \bar{y})+(\bar{x} \ell) \bar{y}-\bar{x}(\ell \bar{y}) \quad \text { (A é alternativa) } \\
& =(x \ell) \bar{y}-(y x) \ell+(\bar{x} \ell) \bar{y}-\bar{x}(y \ell) & (\text { Por } 1.11) \\
& =((x+\bar{x}) \ell) \bar{y}-(y x) \ell-\bar{x}(y \ell) & \\
& =(x+\bar{x})(\ell \bar{y})-\bar{x}(y \ell)-(y x) \ell \quad(x+\bar{x}=T(x) 1 \in \mathbb{F} 1) \\
& =x(y \ell)-(y x) \ell
\end{array}
$$

Portanto,

$$
x(y \ell)=(y x) \ell
$$

para todo $x, y$ em $A_{0}$. Finalmente

$$
\begin{aligned}
(x \bar{y}) \ell & =\bar{y}(x \ell) & & (\text { por relação acima }) \\
& =-\bar{y}(x \ell) & & \left(A_{0}\left(A_{0} \ell\right) \subset A_{0} \ell \mathrm{e} \bar{z}=-z \text { para todo } z \in A_{0} \ell\right) \\
& =-(\overline{x \ell}) y & & (\text { propriedade da involução) } \\
& =(x \ell) y & &
\end{aligned}
$$

Isto termina a demonstração do lema. 
Nós agora podemos enunciar e provar o Teorema de A. Hurwitz.

Teorema 1.2 Sobre um corpo $\mathbb{F}$ de característica diferente de dois, a menos de isomorfismo, o corpo $\mathbb{F} 1, \mathbb{F} 1 \oplus \mathbb{F} 1$, as álgebras generalizadas dos Quatérnios e as álgebras generalizadas dos Octônios, são as únicas álgebras de composição.

\section{Demonstração:}

Seja $A$ uma $F$-álgebra de composição. Se $\mathbb{F} 1$ é uma subálgebra própria de $A$, então $A$ contém uma subálgebra $C_{1}$ que é quadrática e isomorfa à duplicação de $\mathbb{F}$.

Se $C_{1}$ é uma subálgebra própria de $A$, então $A$ contém uma subálgebra generalizada dos Quatérnios $C_{2}$.

Se $C_{2}$ for subálgebra própria de $A$, então obtemos que em $A$ existe uma subálgebra $C_{3}$ isomorfa à álgebra generalizada dos Octônios.

Se $C_{3}$ for uma subálgebra própria de $A$, pelo Lema 1.7, obtemos uma subálgebra $C_{4} \mathrm{em}$ $A$ que é isomorfa à duplicação de $C_{3}$. Mas pelo Lema $1.5, C_{4}$ não é alternativa, ou seja, $A$ contém uma subálgebra não alternativa o que não é possível pelo Lema 1.2. Assim $C_{3}$ não pode ser subálgebra própria de $A$, deve ser a álgebra toda, e paramos na dimensão 8 .

Observemos que na prova anterior não foi assumido que a álgebra de composição fosse de dimensão finita. Portanto, toda álgebra de composição é de dimensão finita e sua dimensão é $1,2,4$ ou 8.

A demonstração dada do teorema de A. Hurwitz está baseada na prova de N. Jacobson em [14] e [15]. Esta prova é obtida através do processo de duplicação de Cayley-Dickson.

Dado que duas álgebras isótopas são iguais como espaços vetoriais, toda $\mathbb{R}$-álgebra induzida por uma solução da equação (1.1) (não necessariamente com unidade), tem dimensão $1,2,4$ ou 8.

\section{$1.2 \quad$ A.a.v. de Dimensão Finita com Unidade}

Nesta seção provamos o Teorema de A. Hurwitz através das álgebras absolutamente valuadas e usando o famoso teorema de F.G. Frobenius. Aqui seguimos as linhas de [1] e [31].

A segunda prova está baseada em [1], onde são consideradas $\mathbb{R}$-álgebras absolutamente valuadas de dimensão finita com unidade. Antes de iniciar, fazemos o seguinte comentário: na primeira prova $\mathbb{F}$ é um corpo qualquer de característica diferente de dois e, nesta prova, consideramos álgebras reais. Mostraremos no Lema 1.11, que a norma, numa álgebra real com unidade absolutamente valuada de dimensão finita, está induzida por um produto interno.

Assumimos nesta seção que $A$ é uma álgebra real com unidade.

Lema 1.8 Sejam A uma álgebra real sem divisores de zero, de dimensão finita e a $\in$ A não escalar, ou seja $a \in A \backslash \mathbb{R} 1$. Então o polinômio característico de $R_{a}$ é produto de fatores quadráticos irredutíveis, isto é não possui raízes reais.

\section{Demonstração:}

Se existir $\alpha$ em $\mathbb{R}$ autovalor de $R_{a}$, então tomando um autovetor correspondente $v \neq 0$, temos que $R_{a}(v)=\alpha v, \operatorname{logo} v(a-\alpha 1)=0$. Como $A$ não tem divisores de zero, $a=\alpha 1$, o que não é possível. Portanto, tal $\alpha$ não existe.

Observemos que uma álgebra real de dimensão finita é sem divisores de zero se, e somente se, é de divisão. De fato, se $A$ é sem divisores de zero, dado $a \in A \backslash\{0\}$ segue que $L_{a}$, 
o operador sobre $A$ multiplicação pela esquerda, é injetor e por ser a álgebra de dimensão finita é sobrejetor. Portanto, para cada $b \in A$ existe um único $x \in A$ tal que $a x=b$. De forma análoga, trocando $L_{a}$ por $R_{a}$ obtemos que para cada $c \in A$, existe um único $x \in A$ tal que $x a=c$. A recíproca é clara.

No seguinte resultado, utilizamos o conceito de complexificação de uma $\mathbb{R}$ álgebra $A$, o qual é definido do seguinte modo.

Definimos a complexificação de uma $\mathbb{R}$-álgebra $A$ como

$$
A_{\mathbb{C}}=\mathbb{C} \otimes_{\mathbb{R}} A .
$$

Em $A_{\mathbb{C}}$ são definidos a multiplicação de elementos e a multiplicação por um número complexo de maneira "natural",

$$
(\lambda \otimes x)\left(\lambda^{\prime} \otimes y\right)=\left(\lambda \lambda^{\prime}\right) \otimes(x y), \quad \lambda\left(\lambda^{\prime} \otimes x\right)=\left(\lambda \lambda^{\prime}\right) \otimes x,
$$

para todo $\lambda, \lambda^{\prime} \in \mathbb{C}$ e $x, y \in A$. É fácil verificar que $A_{\mathbb{C}}$ torna-se, assim, uma álgebra sobre os complexos. Se $\Phi=\left\{a_{1}, \ldots, a_{n}\right\}$ é uma base de $A$, então podemos provar facilmente que $\Phi_{\mathbb{C}}=\left\{1 \otimes a_{1}, \ldots, 1 \otimes a_{n}\right\}$ é uma base de $A_{\mathbb{C}} \cdot \operatorname{Assim}_{\operatorname{dim}_{\mathbb{R}}}(A)=\operatorname{dim}_{\mathbb{C}}\left(A_{\mathbb{C}}\right)$.

Seja $f$ um operador linear de $A$ em $A$. Então sua complexificação $f_{\mathbb{C}}=I \otimes_{\mathbb{R}} f$ é definido como

$$
f_{\mathbb{C}}(\lambda \otimes x)=\lambda \otimes f(x), \quad(\lambda \in \mathbb{C}, \quad x \in A)
$$

é um operador linear de $A_{\mathbb{C}}$ em $A_{\mathbb{C}}$. Se $M \in M_{n}(\mathbb{R})$ for a matriz de $f$ em relação à base $\Phi$, então $M$ também será a matriz de $f_{\mathbb{C}}$ em relação à base $\Phi_{\mathbb{C}}$ de $A_{\mathbb{C}}$. Portanto, $f$ e $f_{\mathbb{C}}$ possuem o mesmo polinômio característico.

Usando as propriedades do produto tensorial, observamos que cada elemento em $A_{\mathbb{C}}$ pode-se exprimir de maneira única na forma

$$
1 \otimes x+i \otimes y
$$

É usual representar este elemento na forma $x+i y$. Com esta notação, temos que

$$
f_{\mathbb{C}}(x+i y)=f(x)+i f(y)
$$

para todo $x, y \in A$. A seguir, no seguinte lema, usaremos esta notação.

Lema 1.9 Seja A uma $\mathbb{R}$-álgebra absolutamente valuada de dimensão finita. Se a $\in$ A, então toda raiz complexa do polinômio característico de $R_{a}$ tem norma $\|a\|$.

\section{Demonstração:}

Seja $\lambda=\rho(\cos \theta+i \sin \theta) \in \mathbb{C}$ uma raiz do polinômio característico do operador $R_{a}$. Então $\lambda$ é um autovalor do operador $f=I \otimes_{\mathbb{R}} R_{a}$. Assim, existe $u=x+y i \neq 0$ em $A_{\mathbb{C}}$, com $\gamma=$ $\|x\|+\|y\| \neq 0$, tal que $f(u)=\lambda u$, logo $f^{m}(u)=\lambda^{m}(u)=\left(\rho^{m}(\cos (m \theta)+i \sin (m \theta))\right)(x+y i)$, para todo inteiro positivo $m$. Por outro lado, $f^{m}(u)=f^{m}(x+y i)=R_{a}^{m}(x)+R_{a}^{m}(y) i$. Portanto,

$$
\left(\begin{array}{c}
R_{a}^{m}(x) \\
R_{a}^{m}(y)
\end{array}\right)=\rho^{m}\left(\begin{array}{cc}
\cos (m \theta) & -\sin (m \theta) \\
\sin (m \theta) & \cos (m \theta)
\end{array}\right)\left(\begin{array}{l}
x \\
y
\end{array}\right) .
$$

Tomando normas obtemos,

$$
\begin{gathered}
\left\|R_{a}^{m}(x)\right\| \leq \rho^{m}(\|x\|+\|y\|) \leq \rho^{m} \gamma \\
\left\|R_{a}^{m}(y)\right\| \leq \rho^{m}(\|x\|+\|y\|) \leq \rho^{m} \gamma .
\end{gathered}
$$


Usando agora que $A$ é absolutamente valuada, segue que

$$
\|a\|^{m}\|x\| \leq \rho^{m} \gamma, \quad\|a\|^{m}\|y\| \leq \rho^{m} \gamma
$$

Somando lado a lado, ambas desigualdades, obtemos que $\|a\|^{m} \leq 2 \rho^{m}$ para todo inteiro positivo $m$. Portanto, $\|a\|=0$ se $\rho=0$. Suponha agora que $\rho \neq 0$, multiplicando pelo inverso de $\rho, m$ vezes, temos que $\|a\|^{m}\left(\rho^{-1}\right)^{m} \leq 2$ para todo inteiro positivo $m$, o que implica que $\|a\| \rho^{-1} \leq 1$, isto é,

$$
\|a\| \leq \rho .
$$

Seja agora $M \in M_{2}(\mathbb{R})$ a matriz que aparece na relação (1.15). Temos que $M$ é uma matriz ortogonal e sua inversa é igual a sua transposta. Assim, multiplicando, ambos membros da igualdade (1.15) por $M^{t}$, pela esquerda, obtemos a igualdade matricial

$$
\rho^{m}\left(\begin{array}{l}
x \\
y
\end{array}\right)=\left(\begin{array}{cc}
\cos (m \theta) & \sin (m \theta) \\
-\sin (m \theta) & \cos (m \theta)
\end{array}\right)\left(\begin{array}{c}
R_{a}^{m}(x) \\
R_{a}^{m}(y)
\end{array}\right) .
$$

Tomando normas, como no caso anterior, obtemos que

$$
\begin{aligned}
& \rho^{m}\|x\| \leq\left\|R_{a}(x)^{m}\right\|+\left\|R_{a}(y)^{m}\right\|=\|a\|^{m}\|x\|+\|a\|^{m}\|y\|=\|a\|^{m} \gamma, \\
& \rho^{m}\|y\| \leq\left\|R_{a}(x)^{m}\right\|+\left\|R_{a}(y)^{m}\right\|=\|a\|^{m}\|x\|+\|a\|^{m}\|y\|=\|a\|^{m} \gamma .
\end{aligned}
$$

Somando as duas desigualdades e multiplicando por $\gamma^{-1}$ temos que $\rho^{m} \leq 2\|a\|^{m}$, logo $\rho^{m}\left(\|a\|^{-1}\right)^{m} \leq 2$ para todo inteiro positivo $m$. Portanto $\rho\|a\|^{-1} \leq 1$, isto é

$$
\rho \leq\|a\|
$$

Isto termina a demonstração do lema.

Observamos que no lema anterior foi utilizado o seguinte fato fácil de provar. Se $\lambda$ é um autovalor de um endomorfismo linear $f$, e $p(X)$ é um polinômio, então $p(\lambda)$ é um autovalor do endomorfismo $p(f)$.

Lema 1.10 Seja a não escalar em uma álgebra real absolutamente valuada de dimensão finita A. Então, o polinômio característico de $R_{a}$ é da forma $\left(X^{2}-\alpha X-\beta\right)^{t}$, onde $X^{2}-\alpha X-\beta$ é um polinômio real quadrático irredutivel.

\section{Demonstração:}

Sejam $\lambda_{1}, \lambda_{2}$ duas raízes complexas, não conjugadas, do polinômio característico de $R_{a}$. Então $\lambda_{1}=\alpha+\beta i$ e $\lambda_{2}=\gamma+\delta i$ com $0<\beta \delta$. Pelo Lema 1.9,

$$
\alpha^{2}+\beta^{2}=\gamma^{2}+\delta^{2}
$$

Por outro lado, $\lambda_{1}+1$ e $\lambda_{2}+1$ são raízes complexas do polinômio característico de $R_{a+1}$, logo pelo lema anterior

$$
(\alpha+1)^{2}+\beta^{2}=(\gamma+1)^{2}+\delta^{2} .
$$

Combinando ambas igualdades, segue que $\alpha=\gamma$ e $\beta^{2}=\delta^{2}$. Como $\beta \delta>0$, podemos concluir que $\beta=\delta$.

Enunciamos sem prova o seguinte resultado, ver [28]. 
Teorema 1.3 [Teorema de Schoenberg] Um espaço normado X é um espaço com produto interno se dados $x$ e y em $X$, com $\|x\|=1=\|y\|$, têm a propriedade de

$$
\|x+y\|^{2}+\|x-y\|^{2} \geq 4 .
$$

Lema 1.11 Se A é uma $\mathbb{R}$-álgebra absolutamente valuada de divisão com elemento unidade, então a norma está induzida por um produto interno.

\section{Demonstração:}

Pelo Teorema 1.3, é suficiente mostrar que

$$
\|x+y\|^{2}+\|x-y\|^{2} \geq 4
$$

para todo $x, y$ em $A \operatorname{com}\|x\|=1$ e $\|y\|=1$. Se $\|x\|=1$, então

$$
\|1+x\|^{2}+\|1-x\|^{2}=\left\|(1+x)^{2}\right\|+\left\|(1-x)^{2}\right\| \geq\left\|(1+x)^{2}-(1-x)^{2}\right\|=\|4 x\|=4 .
$$

Finalmente, sejam $x, y \in A$ de norma 1 . A álgebra $A$ é de divisão, logo o operador multiplicação à direita $R_{x}$ é bijetor e portanto existe $y^{\prime}=R_{x}^{-1}(y)$. Já que $y^{\prime} x=y$, tomando normas podemos afirmar que $\left\|y^{\prime}\right\|=1$. Finalmente

$$
\begin{aligned}
\|x+y\|^{2}+\|x-y\|^{2} & =\left\|\left(1+y^{\prime}\right) x\right\|^{2}+\left\|\left(1-y^{\prime}\right) x\right\|^{2} \\
& =\left\|1+y^{\prime}\right\|^{2}\|x\|^{2}+\left\|1-y^{\prime}\right\|^{2}\|x\|^{2} \\
& =\left\|1+y^{\prime}\right\|^{2}+\left\|1-y^{\prime}\right\|^{2} \geq 4,
\end{aligned}
$$

o que mostra o lema.

Antes da prova do resultado principal desta seção, apresentamos uma demonstração, dada em [12], para o caso associativo, do Teorema de F.G. Frobenius. A prova para o caso de álgebras reais não associativas faz parte do material desenvolvido no trabalho: O Teorema de Frobenius para álgebras não-associativas, [16]. Outra referência para a prova do Teorema de Frobenius para álgebras reais não associativas é [24].

Necessitamos recordar alguns conceitos de álgebras em geral.

Definição 1.2 Seja B uma álgebra sobre um corpo arbitrário $\mathbb{F}$. O comutador e associador em $B$ são definidos por

$$
[x, y]:=x y-y x, \quad(x, y, z):=(x y) z-x(y z) .
$$

O núcleo de $B$, é a parte que associa com todos os elementos, isto é

$$
\operatorname{Nuc}(B)=\{b \in B:(b, x, y)=(x, b, y)=(x, y, b)=0 \text { para todo } x, y \in B\}
$$

e o centro é a parte " escalar" da álgebra que comuta e associa com qualquer elemento da álgebra

$$
\operatorname{Cent}(B)=\{b \in \operatorname{Nuc}(B):[b, x]=0 \text { para todo } x \in B\} .
$$

Uma $\mathbb{F}$-álgebra com unidade chama-se central se seu centro for exatamente $\mathbb{F} 1$.

Lema 1.12 Seja A uma $\mathbb{C}$-álgebra com unidade, associativa, algébrica e sem divisores de zero. Então $A=\mathbb{C}$. 


\section{Demonstração:}

Seja $a \in A$. Como $A$ é algébrica sobre $\mathbb{C}$, a subálgebra $A(a)$ é de dimensão finita. Portanto, existe $p(X)=X^{n}+\alpha_{1} X^{n-1}+\ldots+\alpha_{n-1} X+\alpha_{n}$ mônico em $\mathbb{C}[X]$ tal que $p(a)=0$. O polinômio $p(X)$ pode-se escrever da forma

$$
p(X)=\left(X-\lambda_{1} 1\right)\left(X-\lambda_{2} 1\right) \ldots\left(X-\lambda_{n} 1\right),
$$

onde $\lambda_{1}, \ldots, \lambda_{n}$ são números complexos. Assim $0=p(a)=\left(a-\lambda_{1} 1\right)\left(a-\lambda_{2} 1\right) \ldots\left(a-\lambda_{n} 1\right)$. Como $A$ não possui divisores de zero, obtemos que $\left(a-\lambda_{k} 1\right)=0$ para algum $k, 1 \leq k \leq n$. Assim, $a=\lambda_{k} 1$, ou seja, $A \subseteq \mathbb{C}$. Portanto, $A=\mathbb{C}$.

Teorema 1.4 [Teorema de Frobenius] Seja $A$ uma $\mathbb{R}$-álgebra com unidade, associativa, algébrica e sem divisores de zero. Então $A$ é isomorfa a $\mathbb{R}, \mathbb{C}$ ou $\mathbb{H}$.

\section{Demonstração:}

Suponha que $A \neq \mathbb{R}$ e seja $a \in A \backslash \mathbb{R} 1$. Dado que $A$ é algébrica, $a$ é uma raiz de um polinômio $p(X)$ mônico em $\mathbb{R}[X]$. Como $A$ não possui divisores de zero e $p(X)$ é produto de fatores lineares ou quadráticos em $\mathbb{R}[X]$, temos que $a$ é uma raiz de um fator linear ou de um fator quadrático. Dado que $a \in A \backslash \mathbb{R} 1$, $a$ deve ser raiz de um fator quadrático, portanto, existem $\alpha$ e $\beta$ em $\mathbb{R}$ tais que $a^{2}+2 \alpha a+\beta 1=0$, ou equivalentemente $(a+\alpha 1)^{2}=\left(\alpha^{2}-\beta\right) 1$, com $\alpha^{2}-\beta<0$ pois, no caso contrário, teríamos que $a \in \mathbb{R} 1$. Seja $\gamma \in \mathbb{R}$ positivo tal que $\left(\alpha^{2}-\beta\right)=-\gamma^{2}$. Então $i^{2}=-1$ para $i=(a+\alpha 1) / \gamma$.

Se $A$ é comutativa, então $\mathbb{R}(i)$ é isomorfo ao corpo dos números complexos, e como $A$ é comutativa, $A$ é $\mathbb{R}(i)$-álgebra. Agora pelo Lema 1.12 , segue que $A \cong \mathbb{C}$.

Se $A$ não for central então seu centro $C$ é uma subálgebra comutativa de $A$ de dimensão maior do que 1 , portanto isomorfo a $\mathbb{C}$. Assim $A$ é uma $C$-álgebra e de novo por Lema 1.12, segue que $A \cong \mathbb{C}$.

Suponhamos agora que $A$ é central. Obviamente $i \notin \mathbb{R} 1$, o centro de $A$, logo existe $b \in A$ tal que $c=b i-i b \neq 0$. Um simples cálculo mostra que $i c=i(b i)-i(i b)=i b i+b \mathrm{e}$ $c i=(b i) i-(i b) i=-b-i b i$. Portanto,

$$
i c=-c i \text {. }
$$

$\mathrm{e}$

$$
i c^{2}=(i c) c=-(c i) c=-c(i c)=c(c i)=c^{2} i .
$$

Por outro lado, o elemento $c$ está em $A \backslash \mathbb{R} 1$, logo é uma raiz de um polinômio quadrático em $\mathbb{R}[X]$. Sejam $\lambda$ e $\mu$ em $\mathbb{R}$ tais que $c^{2}+\lambda c+\mu 1=0$. Multiplicando esta relação pela esquerda (e pela direita) por $i$, temos

$$
0=i c^{2}+i(\lambda c)+i \mu=0, \quad c^{2} i+(\lambda c) i+\mu i=0 .
$$

Sabemos que $i$ comuta com $c^{2}$ e $\mu 1$, logo a subtração das relações acima implica

$$
\lambda i c=\lambda c i,
$$

$\operatorname{logo} 0=\lambda(i c-c i)=2 \lambda i c$. Como $A$ não possui divisores de zero, $c i \neq 0$ e portanto $\lambda=0$. Daí, $c^{2}=-\mu 1$. Como $c \notin \mathbb{R} 1$ podemos dizer que $\mu>0$ e escrever $\mu=\nu^{2}$ com $\nu \in \mathbb{R}$, daí $\left(\frac{c}{\nu}\right)^{2}=-1$. Seja $j=\frac{c}{v}$, e $k=i j$. É um simples cálculo mostrar que $1, i, j, k$ são linearmente 
independente e $k^{2}=-1, i j=-j i, i k=-k i=-j, j k=-k j=i$. Assim $H$, a subálgebra de $A$ gerada por $1, i, j, k$, é isomorfa aos quatérnios reais.

Se $a_{1} \in A \backslash \mathbb{R} 1$, então $N\left(a_{1}\right):=\left\{x \in A \mid x a_{1}=a_{1} x\right\}$ é uma subálgebra de $A$ e seu centro contém os elementos 1 e $a_{1}$, portanto $N\left(a_{1}\right)$ não é central. Isto implica, como foi provado acima, que $\mathbb{C} \cong N\left(a_{1}\right)$ e portanto

$$
N\left(a_{1}\right)=\mathbb{R} 1 \oplus \mathbb{R} a_{1}
$$

Suponhamos agora que $H \neq A$ e seja $u \in A \backslash H$. Sabemos que existe $v \in \mathbb{R} 1+\mathbb{R} u$ tal que $v^{2}=-1$. Como $u \notin H$, temos que também $v \notin H$. Um simples cálculo mostra que

$$
\kappa v+v \kappa \in N(\kappa) \cap N(v)=\mathbb{R} 1, \quad(\kappa=i, j, k .)
$$

Sejam $\alpha_{1}, \alpha_{2}, \alpha_{3} \in \mathbb{R}$ tal que $i v+v i=2 \alpha_{1} 1, j v+v j=2 \alpha_{2} 1, k v+v k=2 \alpha_{3} 1$ e definamos

$$
w:=v+\alpha_{1} i+\alpha_{2} j+\alpha_{3} k .
$$

Então $i w+w i=i v+v i+\alpha_{1}\left(2 i^{2}\right)=0$ e da mesma forma temos que $j w+w j=k w+w k=0$. Por outro lado, $0=k w+w k=i j w+w i j=-i w j-i w j=-2 i w j$ o que é uma contradição visto que $A$ não possui divisores de zero. Portanto, $A=H$.

No resultado seguinte, mostraremos que se $A$ é uma $\mathbb{R}$-álgebra de dimensão finita com unidade e absolutamente valuada, então é alternativa o que permite concluir, pelo Teorema de F.G. Frobenius para álgebras não associativas, que $A$ é isomorfa a $\mathbb{R}, \mathbb{C}, \mathbb{H}$ ou $\mathbb{D}$.

Teorema 1.5 Toda álgebra real de dimensão finita com unidade e absolutamente valuada é isomorfa a $\mathbb{R}, \mathbb{C}, \mathbb{H}$ ou $\mathbb{D}$.

\section{Demonstração:}

Mostremos que para todo $a, b$ em $A$ temos que $(a, a, b)=0=(b, a, a)$. Se $a$ é um escalar o resultado é válido.

Se $a$ em $A$ é não escalar, então temos que o polinômio característico de $R_{a}$ é dado por

$$
p(X)=\left(X^{2}-\alpha X-\beta 1\right)^{t},
$$

onde $X^{2}-\alpha X-\beta 1$ é um polinômio real irredutível. Daí, $\left(R_{a}{ }^{2}-\alpha R_{a}-\beta I\right)^{t}=0$.

Nós podemos supor sem perda de generalidade que $\|a\|=1$. Dado que $A$ é absolutamente valuada e de dimensão finita, $R_{a}$ é bijetora e $\left\|R_{a}(x)\right\|=\|x\|\|a\|=\|x\|$, para todo $x$ em $A$. Pelo Lema 1.11, temos que a norma em $A$ está induzida por um produto interno, então $R_{a}$ é um operador normal, desde que é uma isometria bijetora. Portanto, o polinômio minimal de $R_{a}$ é um produto de polinômios mônicos irredutíveis distintos e, pelo Lema 1.10, temos que o minimal deve ser

$$
X^{2}-\alpha X-\beta 1 \text {. }
$$

Logo, $R_{a}^{2}-\alpha R_{a}-\beta I=0$, isto é, para todo $b$ em $A,(b a) a-\alpha b a-\beta b=0$ e daí, $a^{2}-\alpha a-\beta 1=0$. Multiplicando esta última igualdade por um $b$ qualquer à esquerda obtemos, $b a^{2}-\alpha b a-\beta b=$ 0 , então $(b a) a=b a^{2}$, isto é, $(b, a, a)=0$.

Para mostrar que $(a, a, b)=0$ para todo $a, b$ em $A$, observamos que os Lemas $1.8,1.9 \mathrm{e}$ 1.10 são válidos para $L_{a}$, com $a$ elemento não escalar de $A$. Então, o polinômio característico de $L_{a}$ é dado por $p(X)=\left(X^{2}-\alpha_{1} X-\beta_{1} 1\right)^{r}$, onde $X^{2}-\alpha_{1} X-\beta_{1} 1$ é um polinômio real irredutível. Podemos supor de novo, sem perda de generalidade, que $\|a\|=1$, então o 
polinômio minimal de $L_{a}$ é

$$
X^{2}-\alpha_{1} X-\beta_{1} 1
$$

isto é, $L_{a}^{2}-\alpha_{1} L_{a}-\beta_{1} I=0$. Então, para todo $b$ em $A$, temos que $a(a b)-\alpha_{1} a b-\beta_{1} b=0$ donde obtemos que $a^{2}-\alpha_{1} a-\beta_{1}=0$. Multiplicando esta última igualdade por um $b$ qualquer à direita obtém-se que $a^{2} b-\alpha_{1} a b-\beta_{1} b=0$. Portanto, $a^{2} b=a(a b)$, isto é, $(a, a, b)=0$. Isso mostra que $A$ é uma $\mathbb{R}$-álgebra alternativa. Então, pelo Teorema de Frobenius, para $\mathbb{R}$-álgebras alternativas, obtemos que $A$ é isomorfa a $\mathbb{R}, \mathbb{C}, \mathbb{H}$ ou $\mathbb{D}$.

Se $A$ é uma $\mathbb{R}$-álgebra absolutamente valuada de dimensão finita (não necessariamente com unidade) e, $a$ em $A$ é elemento fixo tal que $\|a\|=1$, então o $\mathbb{R}$-espaço vetorial $A$ com o novo produto definido por

$$
x \cdot y=R_{a}^{-1}(x) L_{a}^{-1}(y),
$$

é uma a.a.v onde $a^{2}$ é o elemento unidade. Portanto, pelo Lema 1.11, Teorema 1.5 e Definição 1.1 obtemos o seguinte resultado.

Teorema 1.6 Toda $\mathbb{R}$-álgebra A de dimensão finita e absolutamente valuada é isótopa a $\mathbb{R}$, $\mathbb{C}, \mathbb{H}$ ou $\mathbb{D}$. E, além disso, a norma está induzida por um produto interno.

Terminamos esta seção mostrando que a norma em uma $\mathbb{R}$-álgebra absolutamente valuada de dimensão finita é única.

Definição 1.3 Duas normas $\|\cdot\| e\|\cdot\|_{1}$ sobre um espaço vetorial $X$ são equivalentes se existir números reais positivos $\lambda$ e $\mu$ tais que

$$
\lambda\|x\| \leq\|x\|_{1} \leq \mu\|x\|
$$

para todo elemento $x \in X$.

Este conceito é motivado pelo fato de que normas equivalentes sobre um espaço vetorial $X$ definem a mesma topologia sobre o espaço. Temos o seguinte resultado, ver [18].

Proposição 1.1 Sobre um espaço vetorial de dimensão finita, todas as normas são equivalentes.

Proposição 1.2 Seja A uma $\mathbb{R}$-álgebra de dimensão finita. Suponhamos que $\|\cdot\| e\|\cdot\|_{1}$ são duas normas tal que $A$ é absolutamente valuada com cada uma delas. Então $\|\cdot\|=\|\cdot\|_{1}$.

\section{Demonstração:}

É suficiente mostrar que $\|a\|_{1}=1$ para todo $a \in A$ com $\|a\|=1$. Suponha que existe $a \in A$ tal que $\|a\|=1$ e $\|a\|_{1}=\alpha \neq 1$. A sequência $\left\{a_{n}\right\}_{n \in \mathbb{N}} \operatorname{com} a_{1}=a, a_{2}=a a, a_{n}=a^{n-1} a$, esta contida na bola $S=\{x \in A \mid\|x\| \leq 1\}$. Por ser $A$ de dimensão finita, o conjunto $S$ é compacto, logo existe uma subsequência $\left\{a_{n_{i}}\right\}_{i \in \mathbb{N}}$ que converge para um ponto $a$ em $S$. Por outro lado, a sequência de números reais positivos $\left\{\left\|a_{n_{i}}\right\|\right\}$ converge para 0 se $\alpha<1$ ou para $+\infty$ se $\alpha>1$.

Finalmene, por ser $A$ de dimensão finita $\|\cdot\|$ e $\|\cdot\|_{1}$ são normas equivalentes, $\log 0\|\cdot\|_{1}$ é contínua com relação à topologia induzida por ambas normas. Assim,

$$
\|a\|_{1}=\left\|\lim _{i \rightarrow \infty} a_{n_{i}}\right\|_{1}=\lim _{i \rightarrow \infty}\left\|a_{n_{i}}\right\|_{1}=\infty \text { ou } 0,
$$

que é absurdo. 


\section{$1.3 \quad$ A.a.v. Algébricas com Unidade}

Nesta seção, mostraremos que a hipótese de dimensão finita não é essencial no Teorema 1.5. Primeiramente, mostraremos que toda $\mathbb{R}$-álgebra quadrática, alternativa e sem divisores de zero é de dimensão 1, 2, 4 ou 8. Dito resultado é verdade, com a mesma prova, para um corpo $\mathbb{F}$ de característica diferente de 2. Em seguida mostraremos que toda $\mathbb{R}$-álgebra com unidade, absolutamente valuada e algébrica, é quadrática e alternativa, portanto, de dimensão finita. Assim, podemos aplicar o Teorema 1.5 e obter o resultado principal desta seção, o Teorema 1.8. Nesta seção seguimos as linhas de [2].

Seja $A$ uma álgebra real quadrática, alternativa e sem divisores de zero. Como é quadrática temos que todo $a$ em $A$ é uma raiz de um polinômio quadrático em $\mathbb{R}$. Assim, existem escalares $\alpha$ e $\beta$ em $\mathbb{R}$ tais que $a^{2}-2 \alpha a-\beta 1=0$. Dizemos que a é uma raiz quadrada se é não escalar e $\alpha=0$, ou seja, $a \notin \mathbb{R} 1$ e $a^{2} \in \mathbb{R} 1$. Observemos que se $a$ é uma raiz quadrada então $\beta<0$. Assim, podemos escrever $\beta=-\gamma^{2}$ e então, $\left(\frac{a}{\gamma}\right)^{2}=-1$. Se $a \in A \backslash \mathbb{R} 1$, então $b=a-\alpha 1$ é raiz quadrada, pois, $b^{2}=a^{2}-2 \alpha a+\alpha^{2} 1=\left(-\beta+\alpha^{2}\right) 1 \in \mathbb{R} 1$.

Lema 1.13 Sejam u e v raízes quadradas em uma $\mathbb{R}$-álgebra quadrática $A$ tais que 1 , u e $v$ são linearmente independentes. Então $u v+v u \in \mathbb{R} 1$.

\section{Demonstração:}

Sejam $u^{2}=\alpha 1$ e $v^{2}=\beta 1$ com $\alpha, \beta \in \mathbb{R}$. Como $A$ é quadrática, para cada $t \in \mathbb{R}$ podemos escrever $(u+t v)^{2}=\lambda_{t}(u+t v)+\mu_{t} 1$, onde $\lambda_{t}$ e $\mu_{t}$ são escalares que dependem de $t$. Desenvolvendo $(u+t v)^{2}$ na relação anterior e substituindo $u^{2}$ por $\alpha 1$ e $v^{2}$ por $\beta 1$ obtemos

$$
t(u v+v u)=\lambda_{t} u+t \lambda_{t} v+\left(\mu_{t}-\alpha-t^{2} \beta\right) 1 .
$$

Multiplicando por $t$ a igualdade acima quando $t=1$, temos

$$
t(u v+v u)=t \lambda_{1} u+t \lambda_{1} v+t\left(\mu_{1}-\alpha-\beta\right) 1 .
$$

Subtraindo, ambas relações, pela independência linear de $\{1, u, v\}$, obtemos,

$$
\lambda_{t}=t \lambda_{1}, \quad t \lambda_{t}=t \lambda_{1}
$$

para todo $t \in R$. Portanto $\lambda_{1}=\lambda_{t}=0$ para todo $t$.

Definição 1.4 Dois elementos u e $v$ em A, são ditos J-ortogonais se são diferentes de zero e $o$ seu produto de Jordan é zero, isto é, $u v+v u=0 . O$ conjunto $\left\{u_{1}, \ldots . u_{n}\right\}$ é dito J-ortogonal se $u_{i}$ e $u_{j}$ são J-ortogonais para todo $i \neq j$.

Lema 1.14 Seja $\left\{u_{1}, \ldots, u_{n}\right\}$ um conjunto J-ortogonal em uma $\mathbb{R}$-álgebra quadrática sem divisores de zero. Então o conjunto $\left\{1, u_{1}, \ldots, u_{n}\right\}$ é linearmente independente.

\section{Demonstração:}

Seja $\lambda_{0} 1+\lambda_{1} u_{1}+\ldots+\lambda_{n} u_{n}=0$, com $\lambda_{0}, \lambda_{1}, \ldots, \lambda_{n}$ reais. Se multiplicamos a igualdade anterior pela direita e pela esquerda por $u_{i}$, com $1 \leq i \leq n$, obtemos,

$$
\lambda_{0} u_{i}+\lambda_{1} u_{1} u_{i}+\ldots+\lambda_{i} u_{i}^{2}+\ldots+\lambda_{n} u_{n} u_{i}=0
$$

$\mathrm{e}$

$$
\lambda_{0} u_{i}+\lambda_{1} u_{i} u_{1}+\ldots+\lambda_{i} u_{i}^{2}+\ldots+\lambda_{n} u_{i} u_{n}=0
$$


Como o conjunto $\left\{u_{1}, \ldots, u_{n}\right\}$ é J-ortogonal, somando as duas igualdades anteriores obtemos, $2 \lambda_{0} u_{i}+2 \lambda_{i} u_{i}^{2}=0$, ou seja, $\lambda_{0} u_{i}+\lambda_{i} u_{i}^{2}=0$. Isto implica que $\lambda_{0}=\lambda_{i}=0$ já que $u_{i}$ não é escalar e $u_{i}^{2}$ é um escalar diferente de zero.

Lema 1.15 Seja $\Phi=\left\{u_{1}, \ldots, u_{n}\right\}$ um conjunto J-ortogonal de raízes quadradas em uma $\mathbb{R}$-álgebra $A$ quadrática e sem divisores de zero. Seja $V$ o subespaço vetorial gerado por $\Phi \cup\{1\}$. Se $w \in A \backslash V$, então existe um elemento $v \in V$ tal que $w-v$ é uma raiz quadrada e J-ortogonal com cada elemento de $\Phi$.

\section{Demonstração:}

Seja $w \in A \backslash V$. Como $A$ é quadrática existem números reais $\beta$ e $\gamma$ tais que, $w^{2}=2 \beta w+\gamma 1$. Seja $w^{\prime}=w-\beta 1$. Temos que $\left(w^{\prime}\right)^{2}=w^{2}-2 \beta w+\beta^{2} 1=\left(\gamma+\beta^{2}\right) 1$, logo $w^{\prime}$ é uma raiz quadrada. Dado que $w^{\prime} \notin V$ o conjunto $\left\{1, u_{i}, w^{\prime}\right\}$ é linearmente independente para cada $i$, e pelo Lema $1.13, w^{\prime} u_{i}+u_{i} w^{\prime}=\delta_{i} 1, \operatorname{com} \delta_{i} \in \mathbb{R}$.

Definimos, $u=\frac{1}{2}\left(\alpha_{1}^{-1} \delta_{1} u_{1}+\ldots+\alpha_{n}^{-1} \delta_{n} u_{n}\right)$, onde $u_{i}^{2}=\alpha_{i} 1$. Então,

$$
u u_{i}=\frac{1}{2}\left(\alpha_{1}^{-1} \delta_{1} u_{1} u_{i}+\ldots+\alpha_{n}^{-1} \delta_{n} u_{n} u_{i}\right)
$$

$\mathrm{e}$

$$
u_{i} u=\frac{1}{2}\left(\alpha_{1}^{-1} \delta_{1} u_{i} u_{1}+\ldots+\alpha_{n}^{-1} \delta_{n} u_{i} u_{n}\right) .
$$

Daí, $u u_{i}+u_{i} u=\frac{1}{2} \alpha_{i}^{-1} \delta_{i} u_{i}^{2}+\frac{1}{2} \alpha_{i}^{-1} \delta_{i} u_{i}^{2}=\delta_{i} 1=w^{\prime} u_{i}+u_{i} w^{\prime}$. Portanto $u_{n+1} u_{i}+u_{i} u_{n+1}=0$ para $u_{n+1}=w^{\prime}-u$. Assim, $u_{n+1}$ é J-ortogonal com cada $u_{i}$.

Mostremos finalmente que $u_{n+1}$ é uma raiz quadrada. Primeiro, observamos que $u$ é raiz quadrada, logo Lema 1.13 implica que $u w^{\prime}+w^{\prime} u \in \mathbb{R} 1$. Assim, $u_{n+1}^{2}=\left(w^{\prime}-u\right)^{2}=$ $\left(w^{\prime}\right)^{2}+u^{2}-\left(w^{\prime} u+u w^{\prime}\right) \in \mathbb{R} 1$.

Lema 1.16 Sejam u e $v$ J-ortogonais e raízes quadradas em uma $\mathbb{R}$-álgebra $A$ quadrática, alternativa e sem divisores de zero. Então $H=\mathbb{R} 1+\mathbb{R} u+\mathbb{R} v+\mathbb{R} u v$ é uma subálgebra de $A$ de dimensão quatro sobre $\mathbb{R}$ e é uma álgebra generalizada dos Quatérnios.

\section{Demonstração:}

Temos que $u^{2}=\alpha 1, v^{2}=\beta 1$, com $\alpha$ e $\beta$ números reais não zero e por ser J-ortogonais $u v+v u=0$. Como $A$ é alternativa,

$$
\begin{aligned}
& u(u v)=u^{2} v=\alpha v, \quad(u v) u=-(v u) u=-v u^{2}=-\alpha v, \\
& v(u v)=-v(v u)=-v^{2} u=-\beta u, \quad(u v) v=u v^{2}=\beta u,
\end{aligned}
$$

e usando a identidade de Moufang, temos

$$
(u v)(u v)=-(u v)(v u)=-u v^{2} u=-u^{2} v^{2}=-\alpha \beta .
$$

Em particular, provamos que $\{u, v, u v\}$ é um conjunto J-ortogonal de raízes quadradas, logo por Lema 1.14 sabemos que o conjunto $\{1, u, v, u v\}$ é linearmente independente. Isto termina a demonstração do lema. 
Lema 1.17 Sejam $u_{1}=u, u_{2}=v, u_{3}=u v, u_{4}=w$ uma familia J-ortogonal de raízes quadradas em uma $\mathbb{R}$-álgebra $A$ quadrática, alternativa e sem divisores de zero. Então os elementos $u_{1}, \ldots, u_{4}, u_{5}=u w, u_{6}=v w, u_{7}=(u v) w$ formam um conjunto J-ortogonal de raízes quadradas, isto é, $u_{i}^{2}=\alpha_{i} 1 \in \mathbb{R} 1$, e se $i \neq j, u_{i} u_{j}=\alpha_{i j} u_{k}$, com $\alpha_{i j} \in \mathbb{R}$ e $k$ depende de $i$ e $j$. Além disso, $B=\mathbb{R} 1+\mathbb{R} u_{1}+\ldots+\mathbb{R} u_{7}$ é uma subálgebra de $A$ de dimensão oito.

\section{Demonstração:}

Mostremos que $\left\{u_{1}, \ldots, u_{7}\right\}$ é um conjunto J-ortogonal de raízes quadradas e daremos a tabela do produto de $B$ em relação à base $\left\{1, u_{1}, u_{2} \ldots, u_{7}\right\}$. Por hipóteses do lema, sabemos que $u_{i}$ e raiz quadrada para $1 \leq i \leq 4$. Por lema anterior

$$
\alpha_{3}=-\alpha_{1} \alpha_{2}, \quad u_{1} u_{3}=-u_{3} u_{1}=\alpha_{1} u_{2} \quad \text { e } \quad u_{2} u_{3}=-u_{3} u_{2}=-\alpha_{2} u_{1}
$$

Como $A$ é uma álgebra alternativa, para cada $i \neq j$, com $1 \leq, i, j \leq 3$, temos que

$$
\begin{aligned}
u_{i} u_{4+i} & =u_{i}\left(u_{i} u_{4}\right)=u_{i}^{2} u_{4}=\alpha_{i} u_{4}=u_{4} \alpha_{i}=\left(u_{4} u_{i}\right) u_{i}=-\left(u_{i} u_{4}\right) u_{i}=-u_{i+4} u_{i}, \\
u_{4} u_{4+i} & =u_{4}\left(u_{i} u_{4}\right)=-u_{4}\left(u_{4} u_{i}\right)=-\alpha_{4} u_{i}=-u_{i} \alpha_{4}=-\left(u_{i} u_{4}\right) u_{4}=-u_{4+i} u_{4}, \\
u_{4+i}^{2} & =\left(u_{i} u_{4}\right)\left(u_{i} u_{4}\right)=-\left(u_{4} u_{i}\right)\left(u_{i} u_{4}\right)=-\left(u_{4}\left(u_{i}^{2}\right)\right) u_{4}=\alpha_{i} u_{4}^{2}=\alpha_{i} \alpha_{4}, \\
u_{4+i} u_{4+j} & =\left(u_{i} u_{4}\right)\left(u_{j} u_{4}\right)=-\left(u_{4} u_{i}\right)\left(u_{j} u_{4}\right)=-\left(u_{4}\left(u_{i} u_{j}\right)\right) u_{4}=\left(\left(u_{i} u_{j}\right) u_{4}\right) u_{4} \\
& =\left(u_{i} u_{j}\right) u_{4}^{2}=\left(u_{i} u_{j}\right) \alpha_{4}=\alpha_{4} u_{i} u_{j} .
\end{aligned}
$$

Para determinar os produtos $u_{i}\left(u_{j} u_{4}\right)$ e $\left(u_{j} u_{4}\right) u_{i}$ observamos que

$$
\begin{aligned}
\left(\alpha_{i}+\alpha_{4}\right) u_{j} & =\left(u_{i}+u_{4}\right)^{2} u_{j}=\left(u_{i}+u_{4}\right)\left[\left(u_{i}+u_{4}\right) u_{j}\right]=\left(u_{i}+u_{4}\right)\left(u_{i} u_{j}+u_{4} u_{j}\right) \\
& =\alpha_{i} u_{j}+u_{i}\left(u_{4} u_{j}\right)+u_{4}\left(u_{i} u_{j}\right)+\alpha_{4} u_{j}=\left(\alpha_{i}+\alpha_{4}\right) u_{j}-u_{i}\left(u_{j} u_{4}\right)+u_{4}\left(u_{i} u_{j}\right) .
\end{aligned}
$$

Comparando a primeira e a última expressão das igualdades acima segue que,

$$
u_{i} u_{4+j}=u_{4}\left(u_{i} u_{j}\right)
$$

Como $u_{i} u_{j}=\mathbb{R} u_{i_{j}}$ com $i_{j} \in\{1,2,3\} \backslash\{i, j\}$, segue que $u_{4}\left(u_{i} u_{j}\right)=-\left(u_{i} u_{j}\right) u_{4}$ é múltiplo escalar de $u_{4+i_{j}}$. Analogamente,

$$
\begin{aligned}
u_{j}\left(\alpha_{i}+\alpha_{4}\right) & =u_{j}\left(u_{i}+u_{4}\right)^{2}=\left[u_{j}\left(u_{i}+u_{4}\right)\right]\left(u_{i}+u_{4}\right)=\left(u_{j} u_{i}+u_{j} u_{4}\right)\left(u_{i}+u_{4}\right) \\
& =\alpha_{i} u_{j}+\left(u_{j} u_{i}\right) u_{4}+\left(u_{j} u_{4}\right) u_{i}+\alpha_{4} u_{j}=u_{j}\left(\alpha_{i}+\alpha_{4}\right)+\left(u_{j} u_{i}\right) u_{4}+\left(u_{j} u_{4}\right) u_{i},
\end{aligned}
$$

pela comparação da primeira e última expressão das igualdades acima,

$$
u_{4+j} u_{i}=\left(u_{i} u_{j}\right) u_{4}
$$

Assim, obtemos a seguinte tabela de multiplicação para a família $\left\{1, u_{1}, u_{2}, \ldots, u_{7}\right\}$. 


\begin{tabular}{|c||c|c|c|c||c|c|c|c|}
\hline$\cdot$ & 1 & $u_{1}$ & $u_{2}$ & $u_{3}$ & $u_{4}$ & $u_{5}$ & $u_{6}$ & $u_{7}$ \\
\hline \hline 1 & 1 & $u_{1}$ & $u_{2}$ & $u_{3}$ & $u_{4}$ & $u_{5}$ & $u_{6}$ & $u_{7}$ \\
\hline$u_{1}$ & $u_{1}$ & $\alpha_{1} 1$ & $u_{3}$ & $\alpha_{1} u_{2}$ & $u_{5}$ & $\alpha_{1} u_{4}$ & $-u_{7}$ & $-\alpha_{1} u_{6}$ \\
\hline$u_{2}$ & $u_{2}$ & $-u_{3}$ & $\alpha_{2} 1$ & $-\alpha_{2} u_{1}$ & $u_{6}$ & $u_{7}$ & $\alpha_{2} u_{4}$ & $\alpha_{2} u_{5}$ \\
\hline$u_{3}$ & $u_{3}$ & $-\alpha_{1} u_{2}$ & $\alpha_{2} u_{1}$ & $-\alpha_{1} \alpha_{2} 1$ & $u_{7}$ & $\alpha_{1} u_{6}$ & $-\alpha_{2} u_{5}$ & $-\alpha_{1} \alpha_{2} u_{4}$ \\
\hline \hline$u_{4}$ & $u_{4}$ & $-u_{5}$ & $-u_{6}$ & $-u_{7}$ & $\alpha_{4} 1$ & $-\alpha_{4} u_{1}$ & $-\alpha_{4} u_{2}$ & $-\alpha_{4} u_{3}$ \\
\hline$u_{5}$ & $u_{5}$ & $-\alpha_{1} u_{4}$ & $-u_{7}$ & $-\alpha_{1} u_{6}$ & $\alpha_{4} u_{1}$ & $-\alpha_{1} \alpha_{4} 1$ & $\alpha_{4} u_{3}$ & $\alpha_{1} \alpha_{4} u_{2}$ \\
\hline$u_{6}$ & $u_{6}$ & $u_{7}$ & $-\alpha_{2} u_{4}$ & $\alpha_{2} u_{5}$ & $\alpha_{4} u_{2}$ & $-\alpha_{4} u_{3}$ & $-\alpha_{2} \alpha_{4} 1$ & $-\alpha_{2} \alpha_{4} u_{1}$ \\
\hline$u_{7}$ & $u_{7}$ & $\alpha_{1} u_{6}$ & $-\alpha_{2} u_{5}$ & $\alpha_{1} \alpha_{2} u_{4}$ & $\alpha_{4} u_{3}$ & $-\alpha_{1} \alpha_{4} u_{2}$ & $\alpha_{2} \alpha_{4} u_{1}$ & $\alpha_{1} \alpha_{2} \alpha_{4} 1$ \\
\hline
\end{tabular}

Tabela 1.4: Tabela do produto de B

Finalmente, lema 1.14, implica que o conjunto $\left\{1, u_{1}, \ldots, u_{7}\right\}$ é linearmente independente. Isto termina a demonstração do lema.

Observamos que se no lema anterior, $\alpha_{1}=\alpha_{2}=\alpha_{4}=-1$, a álgebra $B$ corresponde com a álgebra dos Octônios.

Corolário 1.1 Sejam A uma $\mathbb{R}$-álgebra quadrática, alternativa e sem divisores de zero e $\{x, y, z\}$ um conjunto J-ortogonal de raizes quadradas em $A$ tal que z não é elemento da subálgebra gerada pelo conjunto $\{1, x, y\}$. Então $x(y z)=-(x y) z$.

\section{Demonstração:}

Seja $x=u_{1}, y=u_{2}$ e $z=u_{4}$. Então pela tabela anterior temos que

$$
x(y z)=u_{1}\left(u_{2} u_{4}\right)=u_{1} u_{6}=-u_{7}=-u_{3} u_{4}=-\left(u_{1} u_{2}\right) u_{4}=-(x y) z .
$$

Lema 1.18 Sejam $u_{1}=u, u_{2}=v, u_{3}=u v, u_{4}=w$ uma familia J-ortogonal de raízes quadradas em uma $\mathbb{R}$-álgebra $A$ quadrática, alternativa e sem divisores de zero. Então os elementos $u_{1}, \ldots, u_{4}, u_{5}=u w, u_{6}=v w, u_{7}=(u v) w$ formam um conjunto J-ortogonal de raizes quadradas, isto é, $u_{i}^{2}=\alpha_{i} 1 \in \mathbb{R} 1$, e se $i \neq j, u_{i} u_{j}=\alpha_{i j} u_{k}$, com $\alpha_{i j} \in \mathbb{R}$ e $k$ depende de $i$ e $j$. Além disso, $B=\mathbb{R} 1+\mathbb{R} u_{1}+\ldots+\mathbb{R} u_{7}$ é igual à álgebra $A$.

\section{Demonstração:}

Pelo Lema 1.17 somente temos a mostrar que $A=B$. Se $B$ é uma subálgebra própria de $A$, pelo Lema 1.15, existe $l \in A \backslash B$ que é uma raiz quadrada e o conjunto $\left\{u_{1}, u_{2}, \ldots, u_{7}, l\right\}$ é J-ortogonal. Seja $t=(u v)(w l)$. Como $A$ é uma álgebra sem divisores de zero $t \neq 0$.

Mostremos que $t=0$ e obteremos uma contradição. Se aplicamos o Lema 1.17 primeiro ao conjunto $\{u, w, l\}$, depois ao conjunto $\{v, w, l\}$ e finalmente ao conjunto $\{u v, w, l\}$, obtemos que

$$
\{u, v, u v, w, l, w l\},
$$

é um conjunto J-ortogonal de raízes quadradas. Assim, Lema 1.14 implica que

$$
\{1, u, v, u v, w, l, w l\}
$$

é um conjunto linearmente independente. Finalmente 


$$
\begin{aligned}
t & =(u v)(w l)=-[(u v) w] z \quad \text { (por Corolário 1.1 para } x=u v, y=w, z=l) \\
& =[u(v w)] l \quad(\text { por Corolário } 1.1 \text { para } x=u, y=v, z=w) \\
& =-u[(v w) l] \quad \text { (por Corolário 1.1 para } x=u, y=v w, z=l) \\
& =u[v(w l)] \quad(\text { por Corolário 1.1 para } x=v, y=w, z=l) \\
& =-(u v)(w l)=-t .(\quad \text { por Corolário } 1.1 \text { para } x=u, y=v, z=w l)
\end{aligned}
$$

Daí, $t=0$, o que é absurdo, ou seja, tal elemento $l$ não existe e obtemos $A=B$, portanto, a dimensão de $A$ é oito.

Mostramos desse modo o seguinte teorema.

Teorema 1.7 Toda $\mathbb{R}$-álgebra quadrática, alternativa e sem divisores de zero é de dimensão $1,2,4$ ou 8 .

Agora assumimos que $A$ é uma $\mathbb{R}$-álgebra com unidade absolutamente valuada e algébrica. Mostraremos que $A$ é quadrática e alternativa, o que nos permitirá utilizar o Teorema 1.7 e dizer que $A$ tem dimensão finita e, portanto, pelo Teorema $1.5, A$ é isomorfa a $\mathbb{R}, \mathbb{C}, \mathbb{H}$ ou $\mathbb{D}$.

Lema 1.19 Seja A uma $\mathbb{R}$-álgebra absolutamente valuada com 1 e algébrica. Então $A$ é quadrática.

\section{Demonstração:}

Se $a$ em $A$, então $A(a)$ é de dimensão finita e com unidade. Daí, o operador $R_{a}^{\prime}$, a restrição de $R_{a}$ ao subespaço $A(a)$, satisfaz $\left(R_{a}^{\prime}\right)^{2}-\alpha R_{a}^{\prime}-\beta I=0$ para certos escalares $\alpha$ e $\beta$. Portanto, o conjunto $\left\{1, a, a^{2}\right\}$ é linearmente dependente.

Seja $a \in A$ não escalar. Pelo Lema $1.19, \operatorname{dim} A(a)=2$ e agora Teorema 1.5 implica que $A(a) \cong \mathbb{C}$. Por outro lado, sabemos que $z^{2}-2 \Re(z) z+\|z\|^{2}=0$ para cada número complexo z. Assim, pelo isomorfismo $A(a) \cong \mathbb{C}$ de álgebras absolutamente valuadas, temos que existe $\alpha \in \mathbb{R}$ tal que

$$
a^{2}-2 \alpha a+\|a\|^{2} 1=0 .
$$

Já que o elemento $a$ não é escalar, podemos concluir que o polinômio $X^{2}-2 \alpha X+\|a\|^{2} 1$ é irredutível em $\mathbb{R}[X]$. Chama-se polinômio minimo (ou irredutivel) de a sobre $\mathbb{R}$. Se temos que $f(X) \in \mathbb{R}[X]$ é um polinômio que anula $a$, conhecemos que o polinômio mínimo de $a$, divide a $f(X)$.

Lema 1.20 Seja $\left\{u_{1}, \ldots, u_{n}\right\}$ um conjunto J-ortogonal em $A$, tal que $u_{i}^{2}=-1$, para todo $i$. Então $V$, o subespaço normado de A gerado por $\Phi=\left\{1, u_{1}, \ldots, u_{n}\right\}$, é euclideano, isto é, a norma é induzida por um produto escalar. Além do mais, $\Phi$ é uma base ortonormal de $V$.

\section{Demonstração:}

Seja $a \in V$. Existem escalares $\alpha_{0}, \ldots, \alpha_{n}$ tais que $a=\alpha_{0} 1+\alpha_{1} u_{1}+\ldots+\alpha_{n} u_{n}$. Se $a$ é uum escalar, então $a=\alpha_{0} 1$ e $\|a\|=\left\|\alpha_{0} 1\right\|=\left|\alpha_{0}\right|\|1\|=\left|\alpha_{0}\right|$. Observamos, que por ser $A$ uma álgebra absolutamente valuada $\|1\|=\left\|1^{2}\right\|=\|1\|\|1\|$, logo $\|1\|=1$. 
Suponhamos agora que a não é um escalar. Um simples cálculo mostra que

$$
a^{2}-2 \alpha_{0} a+\left(\alpha_{0}^{2}+\alpha_{1}^{2}+\ldots+\alpha_{n}^{2}\right) 1=0 .
$$

Portanto seu polinômio mínimo sobre $\mathbb{R}$ é $X^{2}-2 \alpha_{0} X+\left(\sum_{i=0}^{n} \alpha_{i}^{2}\right)$, e $\|a\|^{2}=\sum_{i=0}^{n} \alpha_{i}^{2}$. Assim, em todos os casos podemos concluir que

$$
\|a\|^{2}=\sum_{i=0}^{n} \alpha_{i}^{2} .
$$

Isto termina a demonstração do lema.

Lema 1.21 Seja A uma $\mathbb{R}$-álgebra absolutamente valuada com 1 e algébrica. Então $A$ é alternativa.

\section{Demonstração:}

É suficiente provar que $(x, x, y)=0=(y, x, x)$ para todo $x, y \in A$. Sejam $a$ e $b$ dois elemento em $A$. Se $a$ ou $b$ são escalares, temos que $(a, a, b)=0=(b, a, a)$ visto que $(1, x, y)=0=$ $(x, 1, y)=(x, y, 1)$ para todo $x, y \in A$. Assumiremos agora que $a$ e $b$ não são escalares. Então, pelo lema anterior e pelo Teorema 1.5, $A(a)$ e $A(b)$ são subálgebras de $A$ isomorfas a $\mathbb{C}$. Portanto, existem elementos $a^{\prime}$ e $b^{\prime}$ de $A$, tais que $A(a)=\left[1, a^{\prime}\right]$ e $A(b)=\left[1, b^{\prime}\right]$, $\left(a^{\prime}\right)^{2}=-1$ e $\left(b^{\prime}\right)^{2}=-1$. Um simples cálculo mostra que $(a, a, b)=0=(b, a, a)$ se e somente se $\left(a^{\prime}, a^{\prime}, b^{\prime}\right)=0=\left(b^{\prime}, a^{\prime}, a^{\prime}\right)$. Assim podemos assumir, sem perda de generalidade, que $a^{2}=-1, b^{2}=-1$ e que o conjunto $\{1, a, b\}$ é linearmente independente.

Pelo Lema 1.13, existe $\delta \in \mathbb{R}$ tal que $a b+b a=\delta 1$. Se desenvolver o produto $(a+t b)^{2}$ podemos deduzir que

$$
(a+t b)^{2}=-\left(t^{2}-t \delta+1\right) 1
$$

para todo $t \in \mathbb{R}$. Para cada $t \in \mathbb{R}$ fixo, deve ser $t^{2}-t \delta+1>0$, pois caso contrário o polinômio $X^{2}+\left(t^{2}-t \delta+1\right) \in \mathbb{R}[X]$ é redutível e implicaria que $a+t b \in \mathbb{R} 1$ em contradição com o fato de ser $\{1, a, b$,$\} linearmente independentes. Assim seu discriminante deve ser negativo,$ isto é $\delta^{2}-4<0$. Se

$$
b_{1}=\frac{1}{\sqrt{4-\delta^{2}}}(\delta a+2 b)
$$

então

$$
\begin{gathered}
a b_{1}+b_{1} a=\frac{1}{\sqrt{4-\delta^{2}}}(-2 \delta+2(a b+b a))=0 \\
b_{1}^{2}=\frac{1}{4-\delta^{2}}\left(-\delta^{2}-4+2 \delta(a b+b a)\right)=\frac{1}{4-\delta^{2}}\left(-4+\delta^{2}\right)=-1 .
\end{gathered}
$$

Observamos agora que $(a, a, b)=(b, a, a)=0$ se e somente se $\left(a, a, b_{1}\right)=\left(b_{1}, a, a\right)=0$. Portanto podemos assumir sem perda de generalidade que $\delta=0$, isto é $a b+b a=0$. Por lema anterior, o subespaço normado $V=\mathbb{R} 1+\mathbb{R} a+\mathbb{R} b$ é euclideano e $\{1, a, b\}$ é uma base ortonormal. Combinando o lema anterior e Lema 1.15, podemos afirmar que o subespaço $W=V+\mathbb{R}(a b)$ é euclideano. Aplicando varias vezes a lei dos cossenos temos

$$
\begin{aligned}
& 2=\|1+b\|^{2}=\|a\|^{2}\|1+b\|^{2}=\|a(1+b)\|^{2}=\|a+a b\|^{2}, \\
& 2=\|1+a\|^{2}=\|1+a\|^{2}\|b\|^{2}=\|(1+a) b\|^{2}=\|b+a b\|^{2}, \\
& 4=\|1+a\|^{2}\|1+b\|^{2}=\|(1+a)(1+b)\|^{2}=\|(1+a+b)+a b\|^{2}, \quad\|1+a+b\|^{2}=3
\end{aligned}
$$


e podemos dizer que $a b$ e ortogonal a $a, b$ e $1+a+b$ respetivamente. Assim, $a b$ é ortogonal a $1, a, b$ e a sequencia $1, a, b, a b$ é uma base ortonormal de $W$. Por outro lado, sabemos por Lema 1.15, que existe $c$ em $W$ de módulo 1 , raiz quadrada, J-ortogonal com $1, a$ e $b$. Como $1=\|c\|=\left\|c^{2}\right\|$, seque que $c^{2}=-1$. Por lema anterior, $\{1, a, b, c\}$ é uma base ortonormal de $W$, que obriga a igualdade $c= \pm a b$. Nós provamos que se $a$ e $b$ são J-ortogonais e raízes quadradas de -1 , então $\{1, a, b, a b\}$ é um conjunto J-ortogonal de raízes quadradas de -1 .

Repetindo o processo para o par $\{a, a b\}$, segue que $\{a, a b, a(a b)\}$ é J-ortogonal e obtemos que $(a(a b))^{2}=-1$. Combinando Lema 1.15 e o lema anterior segue que o subespaço normado $W+\mathbb{R}(a(a b))$ é euclideano e as sequências $\{1, a, b, a b\}$ e $\{1, a, a b, a(a b)\}$ são ortonormais. Agora

$$
4=\|a-1\|^{2}\|b+a b\|^{2}=\|(a-1)(b+a b)\|^{2}=\|-b+a(a b)\|^{2},
$$

implica, pela lei dos cossenos, que

$$
a(a b)=-b .
$$

Consequentemente, $(a, a, b)=a^{2} b-a(a b)=-b+b=0$. A outra relação $(b, a, a)=0$ pode-se provar de maneira análoga.

Desse modo $A$ é uma $\mathbb{R}$-álgebra quadrática, alternativa e absolutamente valuada. Pelo Teorema 1.7, a dimensão de $A$ é finita, o que permite, pelo Teorema 1.5, dar o seguinte resultado.

Teorema 1.8 Se A é uma $\mathbb{R}$-álgebra absolutamente valuada com unidade e algébrica, então $A$ é isomorfa a $\mathbb{R}, \mathbb{C}, \mathbb{H}$ ou $\mathbb{D}$.

\subsection{A.a.v. com Unidade}

Agora mostraremos que a hipótese que $A$ seja algébrica não é essencial no Teorema 1.8. Nesta seção mostramos que toda álgebra real absolutamente valuada com unidade é quadrática e portanto algébrica. Para encerrar essas linhas, damos um exemplo de uma álgebra real absolutamente valuada, sem unidade, de dimensão infinita. O assunto desta seção segue as linhas de [5] e [30].

Lema 1.22 Sejam $A$ uma $\mathbb{R}$-álgebra absolutamente valuada e $B$ um subconjunto de $A$. Se para todo $x, y$ em $B, x y=y x$, então o subespaço gerado por $B,[B]$, é um espaço com produto interno.

\section{Demonstração:}

Para todo $x, y$ em $B$, temos que $(x+y)^{2}=x^{2}+2 x y+y^{2}$ e $(x-y)^{2}=x^{2}-2 x y+y^{2}$. Então $(x+y)^{2}-(x-y)^{2}=4 x y$. Se $\|x\|=1=\|y\|$, segue que $4=\|x y\|=\left\|(x+y)^{2}-(x-y)^{2}\right\| \leq$ $\left\|(x+y)^{2}\right\|+\left\|(x-y)^{2}\right\|=\|x+y\|^{2}+\|x-y\|^{2}$. Pelo Teorema 1.3, obtemos que $[B]$, é um subespaço com produto interno.

Lema 1.23 Sejam A uma $\mathbb{R}$-álgebra absolutamente valuada, $x$ e y elementos da álgebra tais que $\|x\|=\|y\|=1$ e $\|x-y\|=2$. Se $x y=y x$, então $x+y=0$. 


\section{Demonstração:}

Pelo Lema 1.22, o subespaço normado $V=\mathbb{R} x+\mathbb{R} y$ é euclideano. Agora usando a lei dos cossenos, temos

$$
\|x+y\|^{2}+\|x-y\|^{2}=2\left(\|x\|^{2}+\|y\|^{2}\right) .
$$

Assim $\|x+y\|^{2}=0$. Isto termina a demonstração do lema.

O teorema seguinte é devido a Fred B. Wright e Kazimierz Urbanik, ver [30].

Teorema 1.9 [K. Urbanik e F. Wright $]$ Uma $\mathbb{R}$-álgebra A absolutamente valuada com unidade é isomorfa a $\mathbb{R}, \mathbb{C}, \mathbb{H}$ ou $\mathbb{D}$. Portanto, de dimensão 1, 2, 4 ou 8.

\section{Demonstração:}

Mostremos que $A$ é quadrática e o resultado segue então do Teorema 1.8. Provemos que para todo $x$ em $A, x^{2} \in[1, x]$, o que é equivalente a $A(x) \subseteq[1, x]$, ou seja, $\operatorname{dim} A(x) \leq 2$. Se $x$ e 1 são linearmente dependentes, $x=\alpha 1$ e $x^{2}=\alpha x$, portanto, $x^{2} \in[1, x]$.

Assumimos então que o conjunto $\{1, x\}$ é linearmente independente. Como $x$ comuta trivialmente com 1, o subespaço vetorial gerado por 1 e $x$ é euclideano. Assim, usando que $\|1\|=1$, podemos afirmar que existe $u$ em $[1, x]$, tal que $\{1, u\}$ é uma base ortonormal de $[1, x]$. Em particular, temos que $\|\alpha 1+\beta u\|^{2}=\alpha^{2}+\beta^{2}$, para todo $\alpha, \beta \in \mathbb{R}$. Já que $u$ comuta com $1,\left\|1-u^{2}\right\|=\|(1-u)(1+u)\|=\|1-u\|\|1+u\|=\sqrt{(1+1)} \sqrt{(1+1)}=2$ e também $\left\|u^{2}\right\|=\|u\|\|u\|=1$. Pelo Lema 1.23, $1+u^{2}=0$, assim $u^{2}=-1$. Isto implica que [1, $u$ ] é uma subálgebra de $A$ isomorfa a $\mathbb{C}$, o que mostra que $x^{2} \in[1, x]$.

Corolário 1.2 Seja A uma $\mathbb{R}$-álgebra absolutamente valuada, com um elemento idempotente e não nulo, que comuta com todo elemento de A. Então A é de dimensão finita e as dimensões possiveis são 1, 2, 4 ou 8.

\section{Demonstração:}

Mostremos que $A$ é isótopa a uma $\mathbb{R}$-álgebra com unidade. Provemos primeiro que as multiplicações à direita e esquerda $R_{e}$ e $L_{e}$ são sobrejetoras. Seja $x \in A$. Se $x=\alpha e$, então $R_{e}(x)=x=L_{e}(x)$. Se $x \notin \Re$, então $\{e, x\}$ é linearmente independente e por Lema $1.22 \mathrm{o}$ subespaço normado 2-dimensional $[e, x]$ é euclideano. Por outro lado, $\|e\|=\left\|e^{2}\right\|=\|e\|^{2}$ $\operatorname{logo}\|e\|=1$. Portanto existe $u \in[e, x]$ tal que $\{e, u\}$ é uma base ortonormal. Dado que

$$
\left\|e-u^{2}\right\|=\|e-u\|\|e+u\|=\sqrt{(1+1)} \sqrt{(1+1)}=2 \text {, }
$$

podemos afirmar pelo Lema 1.23 que $e+u^{2}=0$. Assim, $u^{2}=-e$ e daí, concluímos que $A(u)=[e, u]=[e, x]$ é isomorfo ao corpo dos complexos. Portanto existem $y, y^{\prime} \in A(u)$ tais que $R_{e}(y)=y e=x$ e $L_{e}\left(y^{\prime}\right)=e y^{\prime}=x$. Consequentemente, $R_{e}$ e $L_{e}$ são sobrejetoras em $A$. Como

$$
\left\|R_{e}(a)\right\|=\left\|L_{e}(a)\right\|=\|e\|\|\mid a\|=\|a\|,
$$

para todo $a \in A$, temos que $R_{a}$ e $L_{a}$ são também injetoras. Portanto são bijetoras e transformam elementos de $A$ em elementos de $A$ com a mesma norma. Assim, as mesmas propriedades são satisfeitas por $R_{a}^{-1}$ e $L_{a}^{-1}$.

Finalmente, o espaço vetorial normado $A$ com novo produto definido por

$$
x \cdot y=R_{e}^{-1}(x) L_{e}^{-1}(y),
$$


é uma álgebra absolutamente valuada, com unidade, já que $\|x \cdot y\|=\left\|R_{e}^{-1}(x) L_{e}^{-1}(y)\right\|=$ $\left\|R_{e}^{-1}(x)\right\|\left\|L_{e}^{-1}(y)\right\|=\|x\|\|\mid y\|$ e $e \cdot x=x \cdot e=x$ para todo $x, y \in A$. Agora por Teorema 1.9, temos o resultado do corolário.

Corolário 1.3 Seja A uma $\mathbb{R}$-álgebra absolutamente valuada de divisão. Então é de dimensão finita e as dimensões possíveis são 1, 2, 4 ou 8.

\section{Demonstração:}

Seja $a \in A$ com $\|a\|=1$, como $A$ é de divisão $R_{a}^{-1}$ e $L_{a}^{-1}$ existem. Para $x, y$ em $A$, definimos um novo produto em $A$ dado por $x \cdot y=R_{a}^{-1}(x) L_{a}^{-1}(y)$. Com este produto, $a^{2}$ é elemento unidade. Pelo Teorema 1.9 e pela Definição 1.1 obtemos o resultado.

Agora damos o exemplo através do qual mostramos que a hipótese, no Teorema 1.9, da unidade é essencial. Na verdade, é um conjunto de exemplos de álgebras absolutamente valuadas de dimensão infinita, [5].

Sejam $U$ um conjunto infinito qualquer e $\varphi: U \times U \rightarrow U$ uma função injetora. Definimos $A$ como sendo o conjunto das funções, $f$, de $U$ em $\mathbb{R}$ tal que $f(u)=0$ para todo $u \in U$ exceto, se for o caso, para um número finito de elementos de $U$. O conjunto $A$ tem estrutura de $\mathbb{R}$-espaço vetorial com as operações naturais:

$$
(f+g)(u):=f(u)+g(u), \quad(\lambda f)(u):=\lambda f(u) \text { para todo } u \in U,
$$

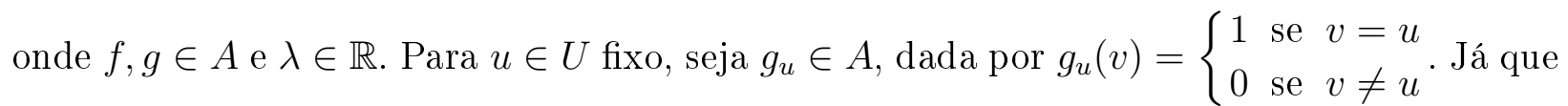
a aplicação de $U$ em $A$, definida por $u \rightarrow g_{u}$, é injetora podemos identificar $g_{u}$ com o elemento $u$. Com esta notação, temos que cada $g \in A$, pode-se exprimir na forma $g=\sum_{u \in U} g(u) u$. Portanto, $U$ gera $A$. De maneira direta, pode-se provar que $U$ é linearmente independente. Portanto $U$ é uma base de $A \operatorname{logo} \operatorname{dim} A=\infty$.

Definimos um produto em $A$ do seguinte modo. Para $f$ e $g$ em $A$,

$$
(f \cdot g)(u):=f(v) g(w)
$$

onde $\varphi(v, w)=u$. Este produto está bem definido pois $\varphi$ é injetora. Com este produto o $\mathbb{R}$-espaço $A$ é uma $\mathbb{R}$-álgebra e para cada $p, 1 \leq p<\infty$, definimos a seguinte norma em $A$ :

$$
\|f\|_{p}=\left(\sum_{u \in U}|f(u)|^{p}\right)^{\frac{1}{p}}
$$

Como

$$
\begin{aligned}
\|f\|_{p}\|g\|_{p} & =\left(\sum_{v \in U}|f(v)|^{p}\right)^{\frac{1}{p}}\left(\sum_{w \in U}|g(w)|^{p}\right)^{\frac{1}{p}}=\left(\sum_{v, w \in U}|f(v) g(w)|^{p}\right)^{\frac{1}{p}} \\
& =\left(\sum_{u \in U}|(f \cdot g)(u)|^{p}\right)^{\frac{1}{p}}=\|f \cdot g\|_{p}
\end{aligned}
$$

para todo $f, g$ em $A$, obtemos que tal álgebra é absolutamente valuada. Assim $A$ é uma álgebra absolutamente valuada de dimensão infinita. Além do mais, se $p \neq 2$, então a norma não é induzida por um produto interno.

O primeiro exemplo de uma álgebra absolutamente valuada de dimensão infinita aparece em [30], que é um caso particular da construção acima, onde $U=\mathbb{N}, p=2$. Como aplicação 
$\varphi$ podemos considerar $\varphi(n, m)=2^{n} 3^{m}$, para $n . m \in \mathbb{Z}$. 


\section{Capítulo 2}

\section{Espaços Normados e Álgebras de Banach}

No capítulo anterior mostramos que uma solução da equação quadrática

$$
\left(\sum_{i=1}^{n} x_{i}^{2}\right)\left(\sum_{i=1}^{n} y_{i}^{2}\right)=\sum_{i=1}^{n} z_{i}^{2}
$$

onde $x_{1}, \ldots, x_{n}, y_{1}, \ldots, y_{n}$ são indeterminadas independentes e $z_{i}=\sum_{j, k=1}^{n} a_{i j k} x_{j} y_{k}$ com $a_{i j k}$ números reais, é equivalente à existência de uma álgebra real absolutamente valuada de dimensão finita (não necessariamente com unidade) com norma induzida por um produto interno. Foi mostrado então que tal álgebra deve ser isótopa a $\mathbb{R}, \mathbb{C}, \mathbb{H}$ ou $\mathbb{D}$. Tal resultado foi obtido através das álgebras de composição, sobre qualquer corpo de característica diferente de dois, e através das álgebras reais absolutamente valuadas de dimensão finita com unidade (Teorema 1.5). O Teorema 1.8, mostra que podemos mudar a hipótese de dimensão finita por algébrica. O Teorema 1.9, prova que podemos prescindir do fato de que a álgebra seja algébrica e finalmente mostramos em um exemplo a existência de uma álgebra real, sem unidade, absolutamente valuadas de dimensão infinita.

O objetivo deste capítulo é apresentar alguns resultados clássicos em análise funcional e alguns outros sobre álgebras de Banach que nos auxiliarão na prova do resultado principal da dissertação. O assunto na seguinte seção segue as linhas de [9], [18] e [31].

\subsection{Espaços Normados}

Um espaço métrico $(X, d)$ é um conjunto $X$ munido de uma métrica (ou distância), isto é, uma função $d: X \times X \rightarrow \mathbb{R}$ tal que para quaisquer $x, y, z \in X:$ (i) $d(x, y)$ é um número real não negativo, (ii) $d(x, y)=0 \Leftrightarrow x=y$, (iii) $d(x, y)=d(y, x)$ (simetria), (iv) $d(x, z) \leq d(x, y)+d(y, z)$ (desigualdade triangular). Um espaço métrico admite uma estrutura natural de espaço topológico. Usando a notação $B(x, r)$ para representar a bola aberta de raio $r, B(x, r):=\{y \mid d(x, y)<r\}$, um conjunto $A$ é aberto quando para cada $x \in A$, existe $\varepsilon>0$ tal que $B(x, \varepsilon) \subset A$. Seja agora $X$ um espaço vetorial sobre o corpo $\mathbb{F}$. Uma norma em $X$ é uma função $\|\cdot\|: X \rightarrow \mathbb{R}^{+}$que satisfaz: i) $\|x\|=0 \Leftrightarrow x=0$; ii) $\|\lambda x\|=|\lambda|\|x\|$, para todo $x \in X$ e todo $\lambda \in \mathbb{F}$; iii) $\|x+y\| \leq\|x\|+\|y\|$ para quaisquer $x, y \in X$ (desigualdade triangular). Um espaço normado é automaticamente um espaço métrico, definindo a métrica $d(x, y):=\|x-y\|$. Porém um espaço métrico pode não ter estrutura algébrica (de espaço vetorial) e portanto o conceito de espaço métrico é uma generalização do conceito de espaço vetorial normado. Uma variação útil da desigualdade 
triangular para espaços normados é

$$
\|x-y\| \geq|\|x\|-\|y\||
$$

para cada par de vetores $x$ e $y$. Esta desigualdade também prova que num espaço normado $(X,\|\cdot\|)$ a função norma $\|\cdot\|: X \rightarrow \mathbb{R}$ é contínua.

Seja $(X, d)$ um espaço métrico. Uma sequência $\left\{x_{n}\right\}$ em $X$ é de Cauchy se para cada $\varepsilon>0$, existir um $n_{0}$ inteiro positivo, que depende de $\varepsilon$, tal que $d\left(x_{n}, x_{m}\right)<\varepsilon$, para todo $n, m \geq n_{0}$. O espaço métrico $(X, d)$ é completo se cada sequência de Cauchy em $X$ converge a um limite em $X$. Falamos que uma sequência $\left\{x_{n}\right\}$ em $X$ converge para $x \in X$ se para cada $\varepsilon>0$, existir um $n_{0}$ inteiro positivo, que depende de $\varepsilon$, tal que $d\left(x_{n}, x\right)<\varepsilon$, para todo $n \geq n_{0}$. Não todos os espaços métricos são completos, más é um fato que todo espaço métrico pode ser "completado", de tal forma que preserve a estrutura essencial do espaço métrico. Se o espaço em questão é um espaço vetorial normado, o processo completa a um espaço de Banach (isto é, espaço normado e completo), e um espaço com produto interno é completado a um espaço de Hilbert (isto é, espaço normado e completo com norma induzida por um produto interno). Se $X$ for uma álgebra normada então podemos estender de maneira natural o produto de $X$ até $\widehat{X}$, de tal forma que $\widehat{X}$ é uma álgebra de Banach.

Nesta seção o nosso resultado principal é o Corolário 2.1 provado em [9].

Teorema 2.1 Dado um espaço métrico $(X, d)$, existe um espaço métrico completo $(\widehat{X}, \hat{d})$ o qual tem um subespaço $W$ que é isométrico a $X$ e é denso em $\widehat{X}$. O espaço $\widehat{X}$ é chamado o completamento de $X$.

\section{Demonstração:}

Seja $\mathcal{S}$ o conjunto de todas as sequências de Cauchy em $X$. Dizemos que dois elementos $\left\{x_{n}\right\}$ e $\left\{y_{n}\right\}$ em $\mathcal{S}$ estão relacionados por " $\sim$ "se

$$
\lim _{n \rightarrow \infty} d\left(x_{n}, y_{n}\right)=0
$$

É fácil ver que " $\sim$ " é uma relação de equivalência sobre $\mathcal{S}$.

Seja então $\widehat{X}$, o conjunto de todas as classes de equivalência. Se $\left\{x_{n}\right\} \in \mathcal{S}$ denotamos por $\left[\left\{x_{n}\right\}\right] \in \widehat{X}$ sua classe de equivalência. Definamos agora uma métrica em $\widehat{X}$ da seguinte forma. Se $\left[\left\{x_{n}\right\}\right]$ e $\left[\left\{y_{n}\right\}\right]$ em $\widehat{X}$, definimos

$$
\hat{d}\left(\left[\left\{x_{n}\right\}\right],\left[\left\{y_{n}\right\}\right]\right)=\lim _{n \rightarrow \infty} d\left(x_{n}, y_{n}\right) .
$$

Observe que $\lim _{n \rightarrow \infty} d\left(x_{n}, y_{n}\right)$ sempre existe, pois temos que

$$
\begin{aligned}
\left|d\left(x_{n}, y_{n}\right)-d\left(x_{m}, y_{m}\right)\right| & \leq\left|d\left(x_{n}, x_{m}\right)+d\left(x_{m}, y_{m}\right)+d\left(y_{m}, y_{n}\right)-d\left(x_{m}, y_{m}\right)\right| \\
& =d\left(x_{n}, x_{m}\right)+d\left(y_{n}, y_{m}\right) \rightarrow 0
\end{aligned}
$$

se $m, n \rightarrow \infty$. Portanto, $\left\{d\left(x_{n}, y_{n}\right)\right\}$ é uma sequência de Cauchy em $\mathbb{R}$, o que implica que $\left\{d\left(x_{n}, y_{n}\right)\right\}$ é convergente. Por outro lado, se $\left\{x_{n}\right\} \sim\left\{x_{n}^{\prime}\right\}$ e $\left\{y_{n}\right\} \sim\left\{y_{n}^{\prime}\right\}$, então

$$
\begin{aligned}
\left|d\left(x_{n}, y_{n}\right)-d\left(x_{n}^{\prime}, y_{n}^{\prime}\right)\right| & \leq\left|d\left(x_{n}, x_{n}^{\prime}\right)+d\left(x_{n}^{\prime}, y_{n}^{\prime}\right)+d\left(y_{n}^{\prime}, y_{n}\right)-d\left(x_{n}^{\prime}, y_{n}^{\prime}\right)\right| \\
& =d\left(x_{n}, x_{n}^{\prime}\right)+d\left(y_{n}, y_{n}^{\prime}\right) \rightarrow 0
\end{aligned}
$$

quando $n$ tende para $\infty$. Portanto, $\hat{d}$ está bem definida, isto é, sempre está definida e o resultado não depende da escolha dos representantes das classes. 
Mostremos que $\hat{d}$ é uma distância em $\widehat{X}$. Sejam $a, b, c \in \widehat{X}$. Por definição $\hat{d}(a, b) \geq 0$ e $\hat{d}(a, b)=0$ se, e somente se, $a=b$ e também por definição $\hat{d}(a, b)=\hat{d}(b, a)$. Como $d$ é uma distância em $X$ temos que $d\left(x_{n}, y_{n}\right) \leq d\left(x_{n}, z_{n}\right)+d\left(z_{n}, y_{n}\right)$, então

$$
\lim _{n \rightarrow \infty} d\left(x_{n}, y_{n}\right) \leq \lim _{n \rightarrow \infty} d\left(x_{n}, z_{n}\right)+\lim _{n \rightarrow \infty} d\left(z_{n}, y_{n}\right) .
$$

Portanto, $\hat{d}(a, b) \leq \hat{d}(a, c)+\hat{d}(c, b)$.

Agora é construída uma isometria $T: X \rightarrow \widehat{X}$. Para cada elemento $x \in X$, seja $\hat{x}$ a classe de equivalência que contém a sequência constante $\left\{x_{n}\right\}$, onde $x_{n}=x$ para todo $n \in \mathbb{N}$. Definimos então $T(x)=\hat{x}$. Temos que $T$ é linear e, por definição, $\hat{d}(T(x), T(y))=\hat{d}(\hat{x}, \hat{y})=$ $\lim _{n \rightarrow \infty} d\left(x_{n}, y_{n}\right)=d(x, y)$. Isto implica que $T$ é uma isometria. Como toda isometria é injetora, se $W=T(X)$, temos que $X$ é isométrico a $W$.

Mostremos agora que $W$ é denso em $\widehat{X}$. Seja $\left[\left\{x_{n}\right\}\right]$ em $\widehat{X}$ e $\varepsilon>0$. Como $\left\{x_{n}\right\}$ é uma sequência de Cauchy, temos que existe $n_{0} \in \mathbb{N}$ tal que $d\left(x_{n}, x_{n_{0}}\right)<\varepsilon$, sempre que $n>n_{0}$. Então $x:=x_{r} \in X$ e $\hat{d}\left(\left[\left\{x_{n}\right\}\right], \hat{x}\right)=\lim _{n \rightarrow \infty} d\left(x_{n}, x\right)<\varepsilon$. Portanto, $W$ é denso em $\widehat{X}$.

O espaço $\widehat{X}$ é completo. Seja $\left\{a_{n}\right\}$ uma sequência de Cauchy em $\widehat{X}$. Como $W$ é denso em $\widehat{X}$, para cada inteiro positivo $n$, existe $x_{n}$ em $X$ tal que $\hat{d}\left(a_{n}, \widehat{x_{n}}\right)<\frac{1}{n}$. Pela desigualdade triangular,

$$
d\left(x_{n}, x_{m}\right)=\hat{d}\left(\widehat{x_{n}}, \widehat{x_{m}}\right) \leq \hat{d}\left(\widehat{x_{n}}, a_{n}\right)+\hat{d}\left(a_{n}, a_{m}\right)+\hat{d}\left(a_{m}, \widehat{x_{m}}\right) \rightarrow 0
$$

quando $n$ e $m$ tendem para $\infty$. Isto implica que $\left\{x_{n}\right\}$ é uma sequência de Cauchy em $X$. Seja $a=\left[\left\{x_{n}\right\}\right] \in \widehat{X}$. Como $\hat{d}\left(a_{n}, \widehat{x_{n}}\right)<\frac{1}{n}$, temos

$$
\hat{d}\left(a_{n}, a\right) \leq \hat{d}\left(a_{n}, \widehat{x_{n}}\right)+\hat{d}\left(\widehat{x_{n}}, a\right) \leq \frac{1}{n}+\hat{d}\left(\widehat{x_{n}}, a\right) .
$$

Por outro lado, para cada $\varepsilon>0$, existe $n_{0}$ tal que $d\left(x_{n}, x_{m}\right)<\varepsilon$ sempre que $n, m>n_{0}$. Assim

$$
\hat{d}\left(\widehat{x_{n}}, a\right)<\varepsilon,
$$

para todo $n>n_{0}$. Significa que o limite da sequência $\hat{d}\left(\widehat{x_{n}}, a\right)$, quando $n$ tende para infinito é zero. Portanto

$$
\lim _{n \rightarrow \infty} \hat{d}\left(a_{n}, a\right)=0
$$

Isto termina a demonstração do teorema.

Suponhamos agora que $X$ é uma álgebra normada. Então $X$, espaço normado, é um espaço métrico com métrica definida por $d(x, y)=\|x-y\|$ para todo $x, y \in X$. Pelo teorema anterior, podemos construir o espaço métrico completo $(\widehat{X}, \hat{d})$. O espaço $\widehat{X}$ tem estrutura de espaço de Banach, via a norma

$$
\left\|\left[\left\{x_{n}\right\}\right]\right\|:=\hat{d}\left(\left[\left\{x_{n}\right\}\right], 0\right)=\lim _{n \rightarrow \infty}\left\|x_{n}\right\| .
$$

Agora $\widehat{X}$ tem estrutura de álgebra normada definindo o seguinte produto. Sejam $a$ e $b$ em $\widehat{X}$. Como $X$ é subespaço denso em $\widehat{X}$, existem sequências $\left\{x_{n}\right\}$ e $\left\{y_{n}\right\}$ em $X$ tais que $x_{n} \rightarrow a$ e $y_{n} \rightarrow b$ e dado que o produto em $X$ é aplicação bilinear contínua segue que a sequência $\left\{x_{n} y_{n}\right\}$ é de Cauchy. Daí, existe $c \in \widehat{X}$ tal que $x_{n} y_{n} \rightarrow c$. Definimos

$$
a b=c=\lim _{n \rightarrow \infty} x_{n} y_{n}
$$


Mostremos que $c$ independe das sequências $\left\{x_{n}\right\}$ e $\left\{y_{n}\right\}$, isto é, se $\left\{x_{n}^{\prime}\right\}$ e $\left\{y_{n}^{\prime}\right\}$ são sequências em $X$ tais que também $x_{n}^{\prime} \rightarrow a$ e $y_{n}^{\prime} \rightarrow b$, então a igualdade

$$
x_{n} y_{n}-x_{n}^{\prime} y_{n}^{\prime}=\left(x_{n}-x_{n}^{\prime}\right) y_{n}+x_{n}^{\prime}\left(y_{n}-y_{n}^{\prime}\right)
$$

mostra que $\lim _{n \rightarrow \infty} x_{n}^{\prime} y_{n}^{\prime}=\lim _{n \rightarrow \infty} x_{n} y_{n}=c$. Além disso, como $\left\|x_{n} y_{n}\right\| \leq\left\|x_{n}\right\|\left\|y_{n}\right\|$ para todo $n$ e a norma é contínua, tomando o limite segue que

$$
\|c\| \leq\|a\|\|b\| .
$$

Por definição, a aplicação $(a, b) \mapsto a b=c$ é bilinear, mostremos que é contínua. Sejam $(a, b)$ e $(c, d)$ dois elementos em $\widehat{X} \times \widehat{X}$ e escrevemos

$$
c d-a b=(c-a)(d-b)+a(d-b)+(c-a) b .
$$

Como $\|a b\| \leq\|a\|\|b\|$ para todo $(a, b)$ em $\widehat{X} \times \widehat{X}$, então dado $\varepsilon>0$, se

$$
\|(c, d)-(a, b)\|:=\|c-a\|+\| d-b \mid \leq \delta=\sqrt{\frac{\varepsilon}{2}},
$$

$\|c d-a b\|=\|(c-a)(d-b)+a(d-b)+(c-a) b\| \leq \frac{\varepsilon}{2}+\frac{\varepsilon}{2}=\varepsilon$, o que mostra a continuidade. Obtemos assim um produto em $\widehat{X}$. Além do mais, se $X$ é uma álgebra associativa o associador em $\widehat{X}$ definido por $(a, b, c)=(a b) c-a(b c)$, é aplicação trilinear contínua, pois o produto é contínuo, e é zero restrito a elementos de $X$ e dado que $X$ é subespaço denso em $\widehat{X}$ obtemos que $(a, b, c)=0$. Então $\widehat{X}$ é também uma álgebra associativa.

Corolário 2.1 Seja $A$ uma $\mathbb{R}$-álgebra absolutamente valuada e suponhamos que existem elementos a e $b$ em $A$ tais que $a A$ e $A b$ são subconjuntos densos em $A$. Então $A$ é de dimensão finita.

\section{Demonstração:}

Podemos supor que $\|a\|=1=\|b\|$ e mostremos que $A$ é isótopa a uma $\mathbb{R}$-álgebra absolutamente valuada com unidade.

Suponhamos que $A$ é um espaço completo. Seja $c$ em $A$. Então existe uma sequência $\left\{a x_{n}\right\}_{n \in \mathbb{N}}$ em $a A$ tal que $a x_{n} \rightarrow c$. A sequência $\left\{x_{n}\right\}_{n \in \mathbb{N}}$ é de Cauchy, já que

$$
\left\|x_{n}-x_{m}\right\|=\left\|a x_{n}-a x_{m}\right\| \rightarrow 0,
$$

quando $n$ e $m$ tendem para $\infty$. Pela completude de $A$, existe $x$ em $A$ tal que

$$
x=\lim _{n \rightarrow \infty} x_{n} .
$$

Por outro lado, em uma álgebra normada e, em particular em uma álgebra absolutamente valuada, o produto é contínuo, logo

$$
a x=a\left(\lim _{n \rightarrow \infty} x_{n}\right)=\lim _{n \rightarrow \infty} a x_{n}=c .
$$

Assim $a A=A$ e a multiplicação pela esquerda por $a$ é bijetora. De maneira análoga podemos provar que $R_{a}$ é bijetora. O $\mathbb{R}$-espaço vetorial $A$ com o novo produto definido por

$$
x \cdot y:=R_{b}^{-1}(x) L_{a}^{-1}(y)
$$


para todo $x, y \in A$, é uma álgebra absolutamente valuada com unidade $e=a b$ :

$$
x \cdot e=R_{b}^{-1}(x) L_{a}^{-1}(a b)=R_{b}^{-1}(x) b=x, \quad e \cdot x=R_{b}^{-1}(a b) L_{a}^{-1}(x)=a L_{a}^{-1}(x)=x .
$$

Pelo Teorema 1.9, obtemos o resultado.

Suponhamos agora que $A$ não é completo e seja $\hat{A}$ o completamento de $A$. Podemos considerar $A$ como subespaço denso de $\hat{A}$. É fácil provar que $a A$ e $A b$ são também densos em $\hat{A}$. Dado que $a A \subseteq a \hat{A}$ e $A b \subseteq \hat{A} b$, podemos afirmar que os conjuntos $a \hat{A}$ e $\hat{A} b$ são densos em $\hat{A}$. Portanto, $\hat{A}$ é isótopa a uma $\mathbb{R}$-álgebra de dimensão finita o que implica que $A$ é de dimensão finita.

Observemos que no lema anterior pode ser $a=b$, fato que é utilizado de forma direta na prova do resultado principal de esta dissertação.

Como no capítulo anterior $\mathbb{F}$ representará o corpo dos números reais ou complexos. Dado um subconjunto $S$ de um espaço normado $X$, representamos por $\bar{S}$ seu fecho topológico em $X$, é dizer o conjunto dos pontos de aderência de $S$ em $X$. Se $X$ e $Y$ forem espaços normados sobre o corpo $\mathbb{F}$, nem toda aplicação linear $T: X \rightarrow Y$ é contínua (em relação as topologias induzidas pelos normas). Para ilustrar esse fato, começamos caracterizando a continuidade de aplicações lineares. São equivalentes as propriedades:

i) Existe $\alpha>0$ tal que $\|T(x)\| \leq \alpha\|x\|$, para todo $x \in X$;

ii) $T$ é lipschitziana: existe $\alpha>0$ tal que $\|T(x)-T(z)\| \leq \alpha\|x-z\|$ para todo $x, z \in X$;

iii) $\mathrm{T}$ é contínua na origem;

iv) $T$ é limitada: $\sup \|T(x)\|=\alpha<\infty$.

Definimos

$$
\|x\| \leq 1
$$

$$
B L(X, Y):=\{T: X \rightarrow Y \mid T \text { é linear e limitada }\} .
$$

É fácil ver que $B L(X, Y)$ é um espaço vetorial normado sobre o corpo $\mathbb{F}$, onde a norma de um operador $T \in B L(X, Y)$, denotada por $\|T\|$, é o número real positivo $\alpha$ definido no item iv). Observamos que este número $\alpha$ coincide com o menor real positivo que satisfaz a desigualdade de i) (ou ii).)

Dizemos que uma aplicação linear $\bar{T}: Z \rightarrow Y$ é uma extensão linear de $T: X \rightarrow Y$ se $X \subset Z$ e $\bar{T}(x)=T(x)$ para todo $x \in X$. Escrevemos $\left.\bar{T}\right|_{X}=T$.

Teorema 2.2 Sejam $X$ um espaço normado, $Y$ um espaço de Banach e $T: D(T) \rightarrow Y$ uma aplicação linear limitada, com $D(T)$ subconjunto de $X$. Então existe $\bar{T}: \overline{D(T)} \rightarrow Y$ uma extensão linear $\bar{T}$ que é limitada e $\|\bar{T}\|=\|T\|$, onde $\overline{D(T)}$ é subconjunto de $\widehat{X}$. Se T for uma isometria, então $\bar{T}$ também é uma isometria.

\section{Demonstração:}

Se $x \in \overline{D(T)}$, então existe uma sequência $\left\{x_{n}\right\}$ de elementos em $D(T)$ que converge para $x$. Dado que $T$ é linear e limitada,

$$
\left\|T\left(x_{n}\right)-T\left(x_{m}\right)\right\| \leq\|T\|\left\|x_{n}-x_{m}\right\| \rightarrow 0,
$$

quando $n$ e $m$ tendem para $\infty$. Portanto a sequência $\left\{T\left(x_{n}\right)\right\}$ é de Cauchy em $Y$. Dado que $Y$ é um espaço de Banach, existe $y \in Y$ tal que a sequência $\left\{T\left(x_{n}\right)\right\}$ converge para $y$. Definimos agora $\bar{T}(x)=y$. Esta definição não depende da sequência convergindo a $x$. De fato, se $\left\{x_{n}\right\} \rightarrow x$ e $\left\{z_{n}\right\} \rightarrow x$, então $v_{n} \rightarrow x$ onde $v_{n}=x_{(n+1) / 2}$ se $n$ ímpar e $v_{n}=z_{n / 2}$ se $n$ par. Podemos provar de maneira análoga que a sequência $\left\{T\left(v_{n}\right)\right\}$ e as subsequências 
$\left\{T\left(x_{n}\right)\right\}$ e $\left\{T\left(y_{n}\right)\right\}$ são de Cauchy logo convergem em $Y$. Portanto, convergem para o mesmo elemento $y$.

A aplicação $\bar{T}$ é linear. Sejam $\alpha$ e $\beta$ em $\mathbb{F}$ e $x, z$ em $\overline{D(T)}$. Existem duas sequências em $D(T)$ que satisfazem $\left\{x_{n}\right\} \rightarrow x$ e $\left\{z_{n}\right\} \rightarrow z$. Então, $\left\{\alpha x_{n}+\beta z_{n}\right\} \rightarrow \alpha x+\beta z$. Assim,

$$
\begin{aligned}
\bar{T}(\alpha x+\beta z) & =\lim _{n \rightarrow \infty} T\left(\alpha x_{n}+\beta z_{n}\right) \quad \text { (por definição) } \\
& =\lim _{n \rightarrow \infty}\left(\alpha T\left(x_{n}\right)+\beta T\left(z_{n}\right)\right) \quad \text { (por ser T linear) } \\
& =\alpha \lim _{n \rightarrow \infty} T\left(x_{n}\right)+\beta \lim _{n \rightarrow \infty} T\left(z_{n}\right) \quad \text { (por existir os limites) } \\
& =\alpha \bar{T}(x)+\beta \bar{T}(z) .
\end{aligned}
$$

Mostraremos agora que $\bar{T}$ é limitada e $\|\bar{T}\|=\|T\|$. Como $\bar{T}$ é uma extensão de $T$, se for limitada $\|\bar{T}\| \geq\|T\|$. Por outro lado, dado $x \in \overline{D(T)}$, existe uma sequência $\left\{x_{n}\right\}$ em $D(T)$ que converge para $x$, logo

$$
\begin{aligned}
\|\bar{T}(x)\| & =\left\|\lim _{n \rightarrow \infty} T\left(x_{n}\right)\right\| & & \text { (por definição de } \bar{T}) \\
& =\lim _{n \rightarrow \infty}\left\|T\left(x_{n}\right)\right\| & & \text { (por ser a função norma contínua) } \\
& \leq \lim _{n \rightarrow \infty}\|T\|\left\|x_{n}\right\| & & \text { (por definição de norma de um operador) } \\
& =\|T\| \lim _{n \rightarrow \infty}\left\|x_{n}\right\| & & \text { (o produto no corpo é contínuo) } \\
& =\|T\|\left\|\lim _{n \rightarrow \infty} x_{n}\right\| & & \text { (por ser a função norma contínua) } \\
& =\|T\|\|x\| . & &
\end{aligned}
$$

Se $T$ for uma isometria, a desigualdade acima é uma igualdade e $\|T\|=1$, logo $\bar{T}$ também é uma isometria. Isto termina a prova do teorema.

Observe que se $D(T)=X$ e $\widehat{X}$ é o completamento de $X$, então existe $\bar{T}: \bar{X}=\hat{X} \rightarrow Y$, uma extensão de $T$, que é linear, limitada e com a mesma norma do que $T$.

Teorema 2.3 Sejam $X, Y$ espaços normados sobre $\mathbb{F}$ e $Y$ um espaço de Banach. Então $B L(X, Y)$ é um espaço de Banach.

\section{Demonstração:}

Seja $\left\{T_{n}\right\}_{n \in \mathbb{N}}$ uma sequência de Cauchy em $B L(X, Y)$. Definiremos uma aplicação $T$ em $B L(X, Y)$ e depois provaremos que dito operador é o limite da sequência. Fixemos agora um elemento $x \in X$. Dado $\varepsilon>0$, existe $n_{0} \in \mathbb{N}$ tal que $\left\|T_{n}-T_{m}\right\|<\varepsilon$, sempre que $n, m>n_{0}$. Logo,

$$
\left\|T_{n}(x)-T_{m}(x)\right\|=\left\|\left(T_{n}-T_{m}\right)(x)\right\| \leq\left\|T_{n}-T_{m}\right\|\|x\|<\varepsilon\|x\|,
$$

sempre que $n, m>n_{0}$. Assim, a sequência $\left\{T_{n}(x)\right\}$ é de Cauchy em $Y$. Uma vez que $Y$ é um espaço de Banach, existe $y \in Y$ tal que $\left\{T_{n}(x)\right\} \rightarrow y$. Definimos $T(x)$ por $y$.

Note que $T$ é linear, pois

$$
T(\alpha x+\beta z)=\lim _{n \rightarrow \infty} T_{n}(\alpha x+\beta z)=\alpha \lim _{n \rightarrow \infty} T_{n}(x)+\beta \lim _{n \rightarrow \infty} T_{n}(z)=\alpha T(x)+\beta T(z),
$$

para todo $\alpha, \beta$ em $\mathbb{F}$ e $x, z$ em $X$.

A aplicação $T$ é também limitada, pois sabemos que $\left\|T_{n}(x)-T_{m}(x)\right\|<\varepsilon\|x\|$, para todo $n, m>n_{0}$ e pela continuidade da norma, segue que para todo $n>n_{0}$ e todo $x \in X$,

$$
\left\|T_{n}(x)-T(x)\right\|=\left\|T_{n}(x)-\lim _{m \rightarrow \infty} T_{m}(x)\right\|=\lim _{m \rightarrow \infty}\left\|T_{n}(x)-T_{m}(x)\right\|<\varepsilon\|x\| .
$$


Assim, $T_{n}-T$ é limitada para todo $n>n_{0}$. Como $T_{n}$ é limitada, $T=T_{n}-\left(T_{n}-T\right)$ é limitada. Desse modo, $T \in B L(X, Y)$.

Finalmente, como $\left\|T_{n}(x)-T(x)\right\|<\varepsilon\|x\|$, sempre que $n>n_{0}$, então para todo $x \in X$ com $\|x\|=1$, obtemos que $\left\|T_{n}-T\right\|<\varepsilon$ e, assim, $T_{n} \rightarrow T$.

Se $Y=\mathbb{F}$ então $B L(X, \mathbb{F})$ é denotado por $X^{\prime}$ e chamado o espaço dual topológico de $X$ e seus elementos são chamados de funcionais lineares. Do teorema anterior temos que $X^{\prime}$ é um espaço de Banach. Usando este último teorema podemos obter uma outra prova de completude para espaços normados bem mais simples: Se $X$ é um espaço normado sobre $\mathbb{F}$, então seu dual $X^{\prime}$ é um espaço de Banach e seu bidual $X^{\prime \prime}:=B L\left(X^{\prime}, \mathbb{F}\right)$ também é um espaço de Banach. Por outro lado podemos definir uma imersão isométrica de $X$ em seu bidual via $x \rightarrow T_{x}$, onde $T_{x}(\varphi)=\varphi(x)$ para todo $\varphi \in X^{\prime}$. Identificando $x$ com $T_{x}$ o completamento de $X$ será seu fecho topológico, considerado como subconjunto do espaço de Banach $X^{\prime \prime}$.

Enunciamos sem prova uma versão do clássico teorema de Hahn-Banach, um resultado central na área de análise funcional.

Teorema 2.4 [Extensão de um funcional linear] Seja $f$ um funcional linear limitado sobre um subespaço $W$ de um espaço normado $X$ sobre $\mathbb{F}$. Então existe um funcional linear limitado $\bar{f}$ sobre $X$ o qual é uma extensão de $f$ a $X$ e tem a mesma norma, isto é, $\|\bar{f}\|=\|f\|$, onde $\|\bar{f}\|=\sup \{|\bar{f}(x)| \mid x \in X$ e $\|x\|=1\}$.

Corolário 2.2 Sejam $X$ um espaço normado sobre $\mathbb{F}$ e $x_{0} \neq 0$ em $X$. Então existe $f \in X^{\prime}$ tal que $\|f\|=1$ e $f\left(x_{0}\right)=\left\|x_{0}\right\|$.

\section{Demonstração:}

Seja $W$ o subespaço de $X$ gerado por $x_{0}$ e $f_{0}$ funcional linear sobre $W$ que satisfaz $f_{0}\left(x_{0}\right)=$ $\left\|x_{0}\right\|$. Temos que $f_{0}$ é limitado e tem norma 1 , pois se $x=\alpha x_{0}$, então $\left|f_{0}(x)\right|=\left|\alpha f_{0}\left(x_{0}\right)\right|=$ $|\alpha|\left\|x_{0}\right\|=\left\|\alpha x_{0}\right\|=\|x\|$. Pelo teorema de Hahn-Banach, $f_{0}$ possui uma extensão $f \in X^{\prime}$ com a mesma norma. Assim $\|f\|=\left\|f_{0}\right\|=1$ e $f\left(x_{0}\right)=f_{0}\left(x_{0}\right)=\left\|x_{0}\right\|$.

Quando $Y=X$ denotamos $B L(X, Y)$ por $B L(X)$. É um simples cálculo, provar que para $T_{1}, T_{2}$ em $B L(X)$,

$$
\left\|T_{1} T_{2}\right\| \leq\left\|T_{1}\right\|\left\|T_{2}\right\|
$$

Além disso, a composição de operadores em $B L(X)$ é uma operação binária associativa, ou seja, $B L(X)$ é uma $\mathbb{F}$-álgebra normada associativa.

\section{2 Álgebra de Banach}

Nesta seção damos alguns resultados sobre álgebras de Banach que precisamos, sendo os Teoremas 2.10 e 2.13, e o Corolário 2.4 os fatos principais. Como na seção anterior $\mathbb{F}$ representa ao corpo dos números reais ou complexos. O assunto desta seção segue as linhas de [6], [9], [10], [18] e [25].

Definição 2.1 Uma $\mathbb{F}$-álgebra A, normada e associativa é dita álgebra de Banach se $A$ como $\mathbb{F}$-espaço normado é completo. 
Por exemplo, $\mathbb{R}$ e $\mathbb{C}$ são $\mathbb{R}$-álgebras de Banach com unidade e comutativas. Também temos que $C[a, b]=\{f:[a, b] \rightarrow \mathbb{R} \mid f$ contínua $\}$ com $\|f\|=\max \{f(t) \mid t \in[a, b]\}$ é uma $\mathbb{R}$-álgebra de Banach com unidade e produto $(f g)(t)=f(t) g(t)$. Se $X$ é um espaço de Banach sobre $\mathbb{F}, B L(X)$ é uma $\mathbb{F}$-álgebra de Banach com unidade. Observamos que em uma álgebra de Banach o produto sempre é contínuo.

Um elemento $x$ em uma álgebra de Banach $A$ com unidade, é dito invertivel ou regular se existe um elemento em $A$, denotado por $x^{-1}$, tal que $x x^{-1}=x^{-1} x=1$. Se $x \in A$ não é regular é dito singular.

Teorema 2.5 Seja A uma $\mathbb{F}$-álgebra de Banach com unidade. Se $x \in X$ satisfaz $\|x\|<1$, então $1-x$ é invertivel e $(1-x)^{-1}=1+\sum_{j=1}^{\infty} x^{j}$.

\section{Demonstração:}

Como $A$ é normada, $\|x y\| \leq\|x\|\|y\|$ para todo $x$ e $y$ em $A$, então $\left\|x^{j}\right\| \leq\|x\|^{j}$ para todo $j$ em $\mathbb{N}$, assim $\sum_{j=1}^{\infty}\left\|x^{j}\right\|$ converge pois $\|x\|<1$. Daí, a série $1+\sum_{j=1}^{\infty} x^{j}$ converge absolutamente e dado que $A$ é um espaço completo, segue que a série converge. Em particular por ser convergente seu termo geral tende para zero isto é, $\lim _{n \rightarrow \infty} x^{n}=0$. Seja $s=1+\sum_{j=1}^{\infty} x^{j}$ e para cada $n$ inteiro positivo $s_{n}=1+\sum_{j=1}^{n} x^{j}$ sua soma parcial. Obviamente, $s=\lim _{n \rightarrow \infty} s_{n}$. Por outro lado,

$$
(1-x) s_{n}=1-x^{j+1}=s_{n}(1-x) .
$$

Assim, usando que o produto em $A$ é contínuo, segue

$$
(1-x) s=(1-x) \lim _{n \rightarrow \infty} s_{n}=\lim _{n \rightarrow \infty}(1-x) s_{n}=\lim _{n \rightarrow \infty}\left(1-x^{n+1}\right)=1,
$$

e analogamente podemos provar que $s(1-x)=1$.

Observe que se $A$ é uma álgebra de Banach com unidade, o conjunto

$$
\operatorname{inv}(A)=\{x \in A \mid x \text { é invertível }\}
$$

é um grupo multiplicativo e temos o seguinte resultado.

Teorema 2.6 Seja A uma álgebra de Banach com unidade sobre $\mathbb{F}$. Se $x_{0}$ é invertivel, então cada elemento da bola aberta $B\left(x_{0},\left\|x_{0}^{-1}\right\|^{-1}\right)$ é invertível. Em particular, o conjunto $\operatorname{inv}(A)$, de todos os elementos regulares de $A$, é um subconjunto aberto em $A$ e portanto $\operatorname{sing}(A)=A \backslash \operatorname{inv}(A)$, o conjunto dos elementos singulares de $A$, é fechado.

\section{Demonstração:}

Seja $x_{0} \in \operatorname{inv}(A)$. Vejamos que $B:=B\left(x_{0},\left\|x_{0}^{-1}\right\|^{-1}\right)$ a bola aberta de centro $x_{0}$ e raio $\left\|x_{0}^{-1}\right\|^{-1}$ está contida em $\operatorname{inv}(A)$. Consideremos um elemento $x$ em $B$. Definamos os elementos

$$
y:=x_{0}^{-1} x \quad \text { e } \quad z:=1-y=1-x_{0}^{-1} x .
$$

Então,

$$
\|z\|=\|y-1\|=\left\|x_{0}^{-1} x-1\right\|=\left\|x_{0}^{-1} x-x_{0}^{-1} x_{0}\right\|=\left\|x_{0}^{-1}\left(x-x_{0}\right)\right\| \leq\left\|x_{0}^{-1}\right\|\left\|x-x_{0}\right\|<1 .
$$

Assim $\|z\|<1$ e o teorema anterior implica que $1-z$ é invertível, isto é, $1-1+y=y$ é um elemento de $\operatorname{inv}(A)$. Como $\operatorname{inv}(A)$ é um grupo multiplicativo,

$$
x_{0} y=x_{0}\left(x_{0}^{-1} x\right)=\left(x_{0} x_{0}^{-1}\right) x=x \in \operatorname{inv}(A) .
$$


Isto prova que $\operatorname{inv}(A)$ é aberto e portanto $\operatorname{sing}(A)=A \backslash \operatorname{inv}(A)$ é fechado.

Definição 2.2 Seja A uma álgebra de Banach com unidade sobre $\mathbb{F}$. O conjunto Resolvente de $x \in A$, denotado por $\rho(x)$, é o conjunto de todos os elementos $\lambda \in \mathbb{F}$ tais que $x-\lambda 1$ é regular. $O$ espectro de $x \in A$, notado por $\sigma(x)$, é o complemento em $\mathbb{F}$ de $\rho(x)$.

Observamos que no caso $\mathbb{F}=\mathbb{R}$ o espectro de um elemento em uma $\mathbb{R}$-álgebra de Banach pode ser vazio. Por exemplo, se $A=B L\left(\mathbb{R}^{2}\right)$ para $T \in A$ dado por $T(x, y)=(-y, x)$, a rotação de grau $\frac{\pi}{2}$, satisfaz $\sigma(T)=\emptyset$. No caso complexo temos o seguinte fato.

Teorema 2.7 Seja A uma álgebra de Banach complexa com unidade. Então para todo $x$ em A, o espectro de $x$ é compacto.

\section{Demonstração:}

Se $|\lambda|>\|x\|$, então $\left\|\lambda^{-1} x\right\|<1$, assim $1-\lambda^{-1} x$ é invertível. Logo, $-\lambda\left(1-\lambda^{-1} x\right)=x-\lambda 1$, é invertível e obtemos que $\lambda \in \rho(x)$. Portanto, se $\lambda \in \sigma(x),|\lambda| \leq\|x\|$. O que mostra que $\sigma(x)$ é limitado.

Vejamos agora que o espectro é fechado. Seja $\lambda_{0}$ em $\mathbb{C} \backslash \sigma(x)=\rho(x)$, isto é, $x-\lambda_{0} 1$ é um elemento invertível em $A$. Por Teorema 2.6, existe uma bola aberta de centro $x-\lambda_{0} 1$ e raio um escalar $\varepsilon>0$ com todos seus elementos inversíveis. Agora, se $\left|\lambda-\lambda_{0}\right|<\varepsilon$, então

$$
\left\|(x-\lambda 1)-\left(x-\lambda_{0} 1\right)\right\|=\left\|\left(\lambda-\lambda_{0}\right) 1\right\|=\left|\lambda-\lambda_{0}\right|<\varepsilon,
$$

e portanto $x-\lambda 1$ é invertível, o que implica que $\lambda \in \rho(x)$. Desse modo $\rho(x)$ é aberto. Assim $\sigma(x)$ é fechado. O que mostra que $\sigma(x)$ é compacto no plano complexo.

Definição 2.3 Seja A uma álgebra normada com unidade sobre $\mathbb{F}$. Um elemento $x$ em $A$ é dito divisor topológico de zero, que denotamos por DTZ, em A se existir uma sequência $\left\{y_{n}\right\}_{n \in \mathbb{N}}$ em $A$ com $\left\|y_{n}\right\|=1$ para todo $n \in \mathbb{N}$ e $\left\{x y_{n}\right\} \rightarrow 0$, (neste caso $x$ é chamado DTZ à esquerda), ou $\left\{y_{n} x\right\} \rightarrow 0$, (neste caso $x$ é dito DTZ à direita). Se existir tal sequência com $\left\{x y_{n}\right\} \rightarrow 0$ e $\left\{y_{n} x\right\} \rightarrow 0, x$ é chamado simplesmente DTZ.

Teorema 2.8 Seja A uma álgebra normada e associativa sobre $\mathbb{F}$. Todo DTZ é singular, isto é, se $x \in A$ é DTZ à esquerda (à direita) então $x$ não tem inverso à esquerda (à direita).

\section{Demonstração:}

Seja $x$ em $A$ tal que tem inverso à esquerda, isto é, existe $y \in A$ tal que $y x=1$, mostremos que $x$ não é DTZ à esquerda. Seja $\left\{y_{n}\right\}_{n \in \mathbb{N}}$ qualquer sequência em $A$ com $\left\|y_{n}\right\|=1$ para todo $n \in \mathbb{N}$. Então, $1=\left\|y_{n}\right\|=\left\|(y x) y_{n}\right\|=\left\|y\left(x y_{n}\right)\right\| \leq\|y\|\left\|x y_{n}\right\|$ o que implica que a sequência $\left\{x y_{n}\right\}_{n \in \mathbb{N}}$ não converge a zero.

Teorema 2.9 Sejam A uma álgebra de Banach sobre $\mathbb{F}$ e $\partial \operatorname{sing}(A)$ a fronteira de $\operatorname{sing}(A)=$ $A \backslash \operatorname{inv}(A)$. Todo elemento de $\partial \operatorname{sing}(A)$ é DTZ. 


\section{Demonstração:}

Seja $x \in \partial \operatorname{sing}(A)=\overline{\operatorname{sing}(A)} \cap \overline{(A \backslash \operatorname{sing}(A))}=\operatorname{sing}(A) \cap \overline{\operatorname{inv}(A)}$, assim $x$ é singular. Como $x \in \overline{\operatorname{inv}(A)}$, existe uma sequência $\left\{x_{n}\right\}_{n \in \mathbb{N}}$ em $\operatorname{inv}(A)$ que converge para $x$. Seja

$$
y_{n}=x_{n}^{-1}, \quad z_{n}=\frac{1}{\left\|x_{n}^{-1}\right\|} x_{n}^{-1} .
$$

Observe que $\left\|z_{n}\right\|=1$, para todo inteiro positivo $n$. Mostraremos agora que $x z_{n} \rightarrow 0$ e $z_{n} x \rightarrow 0$. Primeiro observamos que

$$
1 \leq\left\|1-y_{n} x\right\|
$$

visto que em caso contrário, teríamos por Teorema 2.5 que $y_{n} x=1-\left(1-y_{n} x\right)$ é invertível o que não é possível pois $x$ é singular e $y_{n}$ é regular. Portanto,

$$
1 \leq\left\|1-y_{n} x\right\|=\left\|y_{n} x_{n}-y_{n} x\right\|=\left\|y_{n}\left(x_{n}-x\right)\right\| \leq\left\|y_{n}\right\|\left\|x-x_{n}\right\|,
$$

$\log 0$

$$
\left\|y_{n}\right\|^{-1} \leq\left\|x-x_{n}\right\|
$$

Finalmente,

$$
x z_{n}=\left(x-x_{n}\right) z_{n}+x_{n} z_{n}=\left(x-x_{n}\right) z_{n}+x_{n}\left\|y_{n}\right\|^{-1} y_{n}=\left(x-x_{n}\right) z_{n}+\left\|y_{n}\right\|^{-1} 1,
$$

$\log 0$

$$
\left\|x z_{n}\right\| \leq 2\left\|x-x_{n}\right\| \rightarrow 0,
$$

quando $n$ tende para infinito. De maneira análoga, podemos provar que a sequência $\left\{z_{n} x\right\}$ tende para zero.

Teorema 2.10 Sejam $X$ um espaço normado e $T \in B L(X)$. As seguintes condições sobre $T$ são equivalentes:

i) $T$ é $D T Z$ à esquerda em $B L(X)$;

ii) Existe uma sequência $\left\{x_{n}\right\}_{n \in \mathbb{N}}$ em $X$ tal que $\left\|x_{n}\right\|=1$ para todo $n$ e $\left\{T\left(x_{n}\right)\right\} \rightarrow 0$.

\section{Demonstração:}

i) $\Rightarrow$ ii). Seja $\left\{S_{n}\right\}_{n \in \mathbb{N}}$ uma sequência em $B L(X)$ tal que $\left\|S_{n}\right\|=1$ e $T S_{n} \rightarrow 0$, então $\left\|T S_{n}\right\| \rightarrow 0$. Para cada $n \in \mathbb{N}$, seja $y_{n}$ em $X$ tal que $\left\|y_{n}\right\|=1$ e $\frac{1}{2} \leq\left\|S_{n}\left(y_{n}\right)\right\|$, definimos $x_{n}=\left\|S\left(y_{n}\right)\right\|^{-1} S\left(y_{n}\right)$ para cada $n \in \mathbb{N}$.

$$
\left\|T\left(x_{n}\right)\right\|=\left\|S\left(y_{n}\right)\right\|^{-1}\left\|T\left(S_{n}\left(y_{n}\right)\right)\right\| \leq\left\|S_{n}\left(y_{n}\right)\right\|^{-1}\left\|T S_{n}\right\| \leq 2\left\|T S_{n}\right\|,
$$

mas $T S_{n} \rightarrow 0$, assim $\left\|T\left(x_{n}\right)\right\| \rightarrow 0$ o que implica que $T\left(x_{n}\right) \rightarrow 0$.

ii) $\Rightarrow$ i). Seja $\left\{x_{n}\right\}_{n \in \mathbb{N}}$ uma sequência em $X$ tal que $\left\|x_{n}\right\|=1$ para todo natural e $T\left(x_{n}\right) \rightarrow 0$. Pelo Corolário 2.2, existe $f_{n} \in X^{\prime}$ tal que $\left\|f_{n}\right\|=1$ e $f_{n}\left(x_{n}\right)=\left\|x_{n}\right\|=1$. Para cada $n \in \mathbb{N}$, seja $S_{n} \in B L(X)$ dada por $S_{n}(x)=f_{n}(x) x_{n},\left\|S_{n}\right\|=\left\|f_{n}\right\|\left\|x_{n}\right\|=1$. Além disso, $\left(T S_{n}\right)(x)=T\left(S_{n}(x)\right)=f_{n}(x) T\left(x_{n}\right)$. Portanto, $\left\|T S_{n}\right\|=\left\|f_{n}\right\|\left\|T\left(x_{n}\right)\right\|=\left\|T\left(x_{n}\right)\right\| \rightarrow 0$. Assim, $T S_{n} \rightarrow 0$. 
Teorema 2.11 Sejam $X$ um espaço normado e $T \in B L(X)$. Então $T$ é um divisor de zero à direita se, e somente se, $T(X)$ não é denso em $X$.

\section{Demonstração:}

Suponha que $T(X)$ não é denso em $X$ e seja $y \notin \overline{T(X)}$. Pelo Teorema de Hahn-Banach, existe $f \in X^{\prime}$ tal que $f(y) \neq 0$ e $f=0$ em $T(X)$. Seja $z$ vetor não zero em $X$ e para $x \in X$ definimos $S(x)=f(x) z$. Um simples cálculo prova que $S \in B L(X)$ e temos $(S T)(x)=$ $S(T(x))=f(T(x)) z=0$ para todo $x \in X$. Assim $S T=0$, más $S(y) \neq 0$. Desse modo $T$ é um divisor de zero à direita.

Reciprocamente, seja $T$ divisor de zero à direita, isto é, existe $S \in B L(X), S \neq 0$, tal que $S T=0$. Se $\overline{T(X)}=X, S(T(x))=0$ para todo $x \in X$. Então, $S=0$ o que não é possível.

Teorema 2.12 Sejam $X$ e $Y$ espaços normados sobre $\mathbb{F}$ e $T \in B L(X, Y)$. As seguintes condições são equivalentes:

i) $T$ é limitada inferiormente, isto é, existe um escalar $m>0$ tal que $m\|x\| \leq\|T(x)\|$ para todo $x \in X$;

ii) $T$ é injetora e $T^{-1}: T(X) \rightarrow X$ é limitada;

iii) Não existe uma sequência $\left\{x_{n}\right\}_{n \in \mathbb{N}}$ em $X$, com $\left\|x_{n}\right\|=1$ para todo $n$, tal que $T\left(x_{n}\right) \rightarrow 0$.

\section{Demonstração:}

i) $\Rightarrow$ ii). Se $0 \neq x \in X$, então $0<m\|x\| \leq\|T(x)\|$. Portanto $T$ é injetora e existe $T^{-1}: T(X) \rightarrow X$. Dado $y \in T(X)$, seja $x=T^{-1}(y)$. Então, $m\left\|T^{-1}(y)\right\| \leq\|y\|$, isto é, $\left\|T^{-1}(y)\right\| \leq \frac{1}{m}\|y\|$.

ii) $\Rightarrow$ iii). Se $\left\{x_{n}\right\}_{n \in \mathbb{N}}$ é uma sequência em $X$ tal que $T\left(x_{n}\right) \rightarrow 0$ então pela continuidade de $T^{-1}$ obtemos que $x_{n} \rightarrow 0$.

iii) $\Rightarrow$ i). Suponhamos que não temos $i$ ). Então para cada $n \in N$, existe $y_{n}$ em $X$ tal que $n^{-1}\left\|y_{n}\right\|>\left\|T\left(y_{n}\right)\right\|$, isto é $n\left\|T\left(y_{n}\right)\right\|<\left\|y_{n}\right\|$. Tomemos $x_{n}=\frac{y_{n}}{\left\|y_{n}\right\|}$. Desse modo $\left\|x_{n}\right\|=1$ para todo $n$ e $\left\|T\left(x_{n}\right)\right\|=\frac{1}{\left\|y_{n}\right\|}\left\|T\left(y_{n}\right)\right\| \leq \frac{1}{n}$ o que é absurdo por (iii).

No trabalho [25] encontramos o seguinte resultado que nós enunciamos tendo em conta o Teorema 2.12, ver [10], e o teorema da aplicação aberta.

Teorema 2.13 Sejam $X$ um espaço de Banach sobre $\mathbb{F}$ e $T \in B L(X)$ uma isometria não sobrejetora. Então $B(T, 1)$, a bola aberta em $B L(X)$ com centro $T$ e raio 1 , somente contém operadores limitados inferiormente que não são sobrejetores.

\section{Demonstração:}

Obteremos a prova como caso particular do seguinte resultado geral: se $T$ é um operador linear sobre $X$, limitado inferiormente por $m$ e não sobrejetor, então a bola aberta em $B L(X)$ com centro em $T$ e raio $m$ somente contém operadores limitados inferiormente os quais não são sobrejetores. Para um tal $T$, seja $F \in B L(X)$ que satisfaz $\|T-F\|<m$. Daí,

$$
\begin{aligned}
m\|x\| & \leq\|T(x)\|=\|(T-F)(x)+F(x)\| \leq\|(T-F)(x)\|+\|F(x)\| \\
& \leq\|(T-F)\|\|x\|+\|F(x)\|,
\end{aligned}
$$


donde obtemos que $F$ é limitada inferiormente por $m-\|T-F\|$ e assim $F$ é injetora. Se $\|T-F\|<\frac{m}{2}$ e $F$ é sobrejetora, então $F$ é invertível. Como

$$
(m-\|T-F\|)\|x\| \leq\|F(x)\|,
$$

segue que

$$
(m-\|T-F\|)\left\|F^{-1}(x)\right\| \leq\|x\|,
$$

$\log 0$

$$
\left\|F^{-1}(x)\right\| \leq \frac{1}{m-\|T-F\|}\|x\|<\frac{1}{\frac{m}{2}}\|x\|=\frac{2}{m}\|x\| .
$$

Portanto, $F^{-1} \in B L(X)$, com $\left\|F^{-1}\right\| \leq \frac{1}{m-\|T-F\|}<\frac{2}{m}$. Logo, $\frac{m}{2}<\left\|F^{-1}\right\|^{-1}$ e segue que $\|T-F\|<\left\|F^{-1}\right\|^{-1}$. Pelo Teorema 2.6, obtemos que $T$ é invertível, o que é um absurdo. Então $F$ não é sobrejetora. Portanto a bola aberta em $B L(X)$ de centro $T$ e raio $\frac{m}{2}$ só contém operadores limitados inferiormente os quais não são sobrejetores o que implica que o conjunto

$$
\Omega=\{F \in B L(X) \mid F \text { é limitado inferiormente e não sobrejetor }\}
$$

é aberto em $B L(X)$. Notemos que $B(T, m)$, a bola aberta de centro $T$ e raio $m$, é um espaço topológico conexo o qual é a união disjunta de sua interseção com $\Omega$ e com o conjunto dos elementos inversíveis de $B L(X)$. Dado que essas interseções são abertas e a interseção com $\Omega$ não é vazia, obtemos que a bola $B(T, m)$ está em $\Omega$.

Obtemos o resultado tomando $m=1$, pois para $T \in B L(X)$ isometria, $T$ é limitado inferiormente por 1 .

Corolário 2.3 Sejam $X$ um espaço de Banach sobre $\mathbb{F}$ e $T: X \rightarrow X$ uma isometria não invertivel. Então $\sigma(T)=\{\lambda \in \mathbb{C}|0 \leq| \lambda \mid \leq 1\}$ e se $|\lambda|=1$ então $T-\lambda I \in \partial \operatorname{sing}(B L(X))$.

\section{Demonstração:}

Se $0 \leq|\lambda|<1,\|(T-\lambda I)-T\|=\|\lambda I\|<1$. Então pelo Teorema 2.13, $T-\lambda I$ é singular.

Suponhamos que existe $\lambda_{0}=a+b i$ de norma 1 , tal que $T-\lambda_{0} I \in \operatorname{inv}(B L(X))$. O Teorema 2.6 mostra que $\operatorname{inv}(B L(X))$ é aberto, então existe $\delta>0$ tal que $B\left(T-\lambda_{0} I, \delta\right) \subseteq \operatorname{inv}(B L(X))$. Se $\lambda \in \mathbb{F}$ é tal que $0<|\lambda|<\delta$, então

$$
\left\|T-\left(\lambda_{0}-\lambda\right) I-\left(T-\lambda_{0} I\right)\right\|=\|\lambda I\|=|\lambda|<\delta,
$$

e portanto $T-\left(\lambda_{0}-\lambda\right) I$ é invertível. Porém, podemos achar um $\lambda$ de módulo menor do que $\delta$ tal que $\left|\lambda_{0}-\lambda\right|<1$, o que implicaria que $T-\left(\lambda_{0}-\lambda\right) I$ singular, o que é um absurdo. Portanto, tal $\lambda_{0}$ não existe e segue que se $|\lambda|=1, T-\lambda I$ é singular.

Pelo Teorema 2.5, temos que se $|\alpha|>1$ então $T-\alpha I=(-\alpha)\left(I-\alpha^{-1} T\right)$ é regular pois $\|T\|=1$. Se $|\lambda|=1,\left|\lambda+\frac{1}{n}\right|>1$ para todo $n \in \mathbb{N}$. Então, $T-\left(\lambda+\frac{1}{n}\right) I$ é regular. Mas $T-\left(\lambda+\frac{1}{n}\right) I \rightarrow T-\lambda I$. O que mostra que $T-\lambda I \in \partial \operatorname{sing}(B L(X))$.

Observemos que pelo Teorema 2.9, se $|\lambda|=1$ então $T-\lambda I$ é um DTZ.

Corolário 2.4 Seja $X$ um espaço normado sobre $\mathbb{F}$ e $T: X \rightarrow X$ uma isometria tal que $T(X)$ não é denso. Então existe uma sequência $\left\{x_{n}\right\}_{n \in \mathbb{N}}$ em $X$, com $\left\|x_{n}\right\|=1$ para todo $n \in \mathbb{N}$, tal que $\left\{T\left(x_{n}\right)-x_{n}\right\} \rightarrow 0$. 


\section{Demonstração:}

Assumimos primeiro que $X$ é um espaço completo. Sabemos por Teorema 2.3 que $B L(X)$ é uma álgebra de Banach. Pelo Corolário 2.3, temos que $1 \in \sigma(T)$ e que $T-I \in \partial \operatorname{sing}(B L(X))$. Então pelo Teorema 2.9, $T-I$ é um DTZ e é em particular um DTZ à esquerda e assim pelo Teorema 2.10, existe uma sequência $\left\{x_{n}\right\}_{n \in \mathbb{N}}$ em $X$,com $\left\|x_{n}\right\|=1$ para todo $n \in \mathbb{N}$, tal que $\left\{T\left(x_{n}\right)-x_{n}\right\} \rightarrow 0$.

Se $X$ não é completo, seja $\hat{X}$ o completamento de $X$. Considerando $X$ subespaço (denso) de $\hat{X}$, pelo Teorema 2.2, existe uma isometria $\bar{T}: \hat{X} \rightarrow \hat{X}$ que é uma extensão de $T$. Observando como foi definido $\bar{T}$ em Teorema 2.2, sabemos que a imagem do operador $\bar{T}$ está contida no fecho topológico de $T(X)$. Como $T(X)$ não é denso em $\hat{X}$, temos então que $\bar{T}(\hat{X})$ não é denso em $\hat{X}$. Pela primeira parte da prova sabemos que existe uma sequência $\left\{y_{n}\right\}_{n \in \mathbb{N}}$ em $\hat{X}$, com $\left\|y_{n}\right\|=1$ para todo $n \in \mathbb{N}$, tal que $\left\{\bar{T}\left(y_{n}\right)-y_{n}\right\} \rightarrow 0$. Como $X$ é denso em $\hat{X}$, podemos tomar para cada $n \in \mathbb{N}, x_{n} \in X,\left\|y_{n}-x_{n}\right\|<\frac{1}{2 n}$. Assim

$$
1-\frac{1}{2 n}<\left\|x_{n}\right\|<1+\frac{1}{2 n}
$$

$\log 0$

$$
\begin{aligned}
\left\|y_{n}-\frac{x_{n}}{\left\|x_{n}\right\|}\right\| & =\left\|y_{n}-x_{n}+x_{n}-\frac{x_{n}}{\left\|x_{n}\right\|}\right\|=\left\|\left(y_{n}-x_{n}\right)+\frac{\left(\left\|x_{n}\right\|-1\right) x_{n}}{\left\|x_{n}\right\|}\right\| \\
& \leq\left\|\left(y_{n}-x_{n}\right)\right\|+\left\|\frac{\left(\left\|x_{n}\right\|-1\right) x_{n}}{\left\|x_{n}\right\|}\right\|<\frac{1}{2 n}+\frac{1}{2 n}=\frac{1}{n} .
\end{aligned}
$$

Seja $z_{n}=x_{n} /\left\|x_{n}\right\|$. Temos que $\left\{z_{n}\right\}$ é uma sequência em $X$ de elementos de norma 1 que satisfaz $\left\{y_{n}-z_{n}\right\} \rightarrow 0$ e

$$
\begin{aligned}
& \lim _{n \rightarrow \infty}\left(T\left(z_{n}\right)-z_{n}\right)=\lim _{n \rightarrow \infty}\left(\bar{T}\left(z_{n}-y_{n}+y_{n}\right)-z_{n}+y_{n}-y_{n}\right) \\
& \quad=\lim _{n \rightarrow \infty}\left(\bar{T}\left(z_{n}-y_{n}\right)+\bar{T}\left(y_{n}\right)-\left(z_{n}-y_{n}\right)-y_{n}\right) \quad \text { (por ser } \bar{T} \text { linear) } \\
& \quad=\lim _{n \rightarrow \infty}\left(\bar{T}\left(z_{n}-y_{n}\right)-\left(z_{n}-y_{n}\right)+\left(\bar{T}\left(y_{n}\right)-y_{n}\right)\right) \\
& =\lim _{n \rightarrow \infty} \bar{T}\left(z_{n}-y_{n}\right)-\lim _{n \rightarrow \infty}\left(z_{n}-y_{n}\right)+\lim _{n \rightarrow \infty}\left(\bar{T}\left(y_{n}\right)-y_{n}\right) \quad \text { (por existir todos os limites) } \\
& \quad=\bar{T}\left(\lim _{n \rightarrow \infty}\left(z_{n}-y_{n}\right)\right)-0+0=0 \quad \text { (por ser } \bar{T} \text { contínua) }
\end{aligned}
$$

Isto termina a demonstração do corolário.

O Teorema 2.13 e, os Corolários 2.3 e 2.4, são obtidos em [10] aplicando técnicas dos operadores de Fredholm.

\subsection{Diferenciabilidade da Norma}

Nesta seção mostramos que toda $\mathbb{R}$-álgebra absolutamente valuada algébrica é Fréchet diferenciável em um certo conjunto aberto e denso na álgebra. Os resultados principais da seção são o Teorema 2.15 e a Proposição 2.3. O assunto desta seção segue as linhas dos livros, [15], [19], [21] e, do artigo [9].

Definição 2.4 Sejam $X$ e $Y$ espaços normados sobre $\mathbb{R}, U \subseteq X$ subconjunto aberto de $X$ 
e $f: U \rightarrow Y$ uma função. Dado $x \in U$, se existir $T \in B L(X, Y)$ tal que

$$
\lim _{h \rightarrow 0} \frac{\|f(x+h)-f(x)-T(h)\|}{\|h\|}=0
$$

com $h \in X$ tal que $x+h \in U$, então falamos que $f$ é Fréchet diferenciável em $x$ ou simplesmente diferenciável em $x$. Neste caso $T$ é chamada de derivada de Fréchet ou derivada de $f$ e denota-se por $f^{\prime}(x)$.

Dado $x \in U$ e $v \in X$, dizemos que $f$ é diferenciável na direção $v$ se

$$
\lim _{t \rightarrow 0} \frac{f(x+t v)-f(x)}{t}
$$

existe. Quando o limite existir para todo $v \in X$, falamos que $f$ é Gateaux diferenciável em $x$.

Proposição 2.1 Sejam $X$ e $Y$ espaços normados sobre $\mathbb{R}, U \subseteq X$ aberto em $X$ e $f: U \rightarrow Y$ diferenciável em $x \in U$, então $T \in B L(X, Y)$ dada na Definição 2.4 é única.

\section{Demonstração:}

Sejam $T_{1}$ e $T_{2}$ em $B L(X, Y)$ como na definição anterior. Então para cada $\varepsilon>0$ existe $\delta>0$ tal que se $\|h\|<\delta$ temos que $\left\|f(x+h)-f(a)-T_{i}(h)\right\|<\varepsilon\|h\|$ para $i=1$, 2. Pela desigualdade triangular temos que $\left\|\left(T_{1}-T_{2}\right)(h)\right\|=\left\|T_{1}(h)-T_{2}(h)\right\| \leq 2 \varepsilon\|h\|$ sempre que $\|h\|<\delta$, logo $\left\|T_{1}-T_{2}\right\| \leq 2 \varepsilon$. Dado que $\varepsilon$ é um número real positivo arbitrário, obtemos que $\left\|T_{1}-T_{2}\right\|=0$, isto é $T_{1}=T_{2}$.

Proposição 2.2 Sejam $X$ e $Y$ espaços normados sobre $\mathbb{R}, U \subseteq X$ aberto em $X$ e $f: U \rightarrow Y$ diferenciável em $x \in U$. Então $f$ é contínua em $x$.

\section{Demonstração:}

Denotemos por $T$ a derivada de de Fréchet de $f$ em $x$. Seja $\varepsilon>0$. Como $f$ é diferenciável, temos que existe $\delta>0$ tal que

$$
\frac{\|f(x+h)-f(x)-T(h)\|}{\|h\|}<1
$$

se $0<\|h\|<\delta$. Seja agora

$$
\delta_{1}=\min \left\{\delta, \frac{\varepsilon}{1+\|T\|}\right\}
$$

Pela desigualdade triangular obtemos $\|f(x+h)-f(x)\| \leq\|f(x+h)-f(x)-T(h)\|+\|T(h)\|<$ $\|h\|+\|T(h)\| \leq\|h\|+\|T\|\|h\|<\delta(1+\|T\|)<\varepsilon$, sempre que $0<\|h\|<\delta_{1}$. Portanto $f$ é contínua em $x$.

Teorema 2.14 Sejam $X$ e $Y$ espaços normados sobre $\mathbb{R}, U$ subconjunto aberto de $X, f, g$ : $U \rightarrow Y$ funções Fréchet diferenciáveis em $x \in U$. Então:

i) Para todo $\alpha \in \mathbb{R}$ a função $\alpha f+g$ é Fréchet diferenciável em $x, e$

$$
(\alpha f+g)^{\prime}(x)=\alpha f^{\prime}(x)+g^{\prime}(x)
$$


ii) $S e Y=\mathbb{R}$, então $f g: U \rightarrow \mathbb{R}$ é Fréchet diferenciável em $x$ e

$$
(f g)^{\prime}(x)=f(x) g^{\prime}(x)+g(x) f^{\prime}(x)
$$

iii) Se $Y=\mathbb{R}$ e $f(x) \neq 0$, então existe $V$ aberto de $X$ com $x \in V \subseteq U$, tal que a aplicação $\frac{1}{f}: V \rightarrow \mathbb{R}$ é Fréchet diferenciável em $x$ e

$$
\left(\frac{1}{f}\right)^{\prime}(x)=-\frac{1}{(f(x))^{2}} f^{\prime}(x)
$$

\section{Demonstração:}

i) Temos pela Desigualdade Triangular

$$
\begin{aligned}
& \lim _{h \rightarrow 0} \frac{\left\|(\alpha f+g)(x+h)-(\alpha f+g)(x)-\left(\alpha f^{\prime}(x)+g^{\prime}(x)\right)(h)\right\|}{\|h\|} \\
& \quad \leq \lim _{h \rightarrow 0} \frac{\left\|\alpha f(x+h)-\alpha f(x)-\alpha f^{\prime}(x)(h)\right\|+\left\|g(x+h)-g(x)-g^{\prime}(x)(h)\right\|}{\|h\|} \\
& \quad=\alpha \lim _{h \rightarrow 0} \frac{\left\|f(x+h)-f(x)-f^{\prime}(x)(h)\right\|}{\|h\|}+\lim _{h \rightarrow 0} \frac{\left\|g(x+h)-g(x)-g^{\prime}(x)(h)\right\|}{\|h\|}=0
\end{aligned}
$$

Agora, pela unicidade da derivada obtemos o resultado.

ii) Por simplicidade, introduzimos as notações $\Delta t:=t(x+h)-t(x)$, para uma função $t$. Representamos por $F$ e $G$ os operadores $f^{\prime}(x)$ e $g^{\prime}(x)$ respetivamente. Temos a relação

$$
\begin{aligned}
\Delta(f g) & =f(x+h) g(x+h)-f(x) g(x)+f(x+h) g(x)-f(x+h) g(x) \\
& =f(x+h) \Delta g+g(x) \Delta f,
\end{aligned}
$$

e também

$$
\begin{aligned}
& f(x+h) \Delta g+g(x) \Delta f-[f(x) G(h)+g(x) F(h)] \\
& =f(x+h) \Delta g+g(x) \Delta f-f(x) G(h)-g(x) F(h)+f(x+h) G(h)-f(x+h) G(h) \\
& =f(x+h)[\Delta g-G(h)]+g(x)[\Delta f-F(h)]+\Delta f G(h) .
\end{aligned}
$$

Portanto

$$
\begin{aligned}
& \lim _{h \rightarrow 0} \frac{\|\Delta(f g)-[f(x) G(h)+g(x) F(h)]\|}{\|h\|} \\
& \leq \lim _{h \rightarrow 0}\|f(x+h)\| \cdot \lim _{h \rightarrow 0} \frac{\|\Delta g-G(h)\|}{\|h\|}+\|g(x)\| \cdot \lim _{h \rightarrow 0} \frac{\|\Delta f-F(h)\|}{\|h\|}+\lim _{h \rightarrow 0}\|\Delta f\| \frac{\|G(h)\|}{\|h\|} \\
& \leq\|f(x)\| \cdot 0+\|g(x)\| \cdot 0+0=0,
\end{aligned}
$$

pois por ser $f$ contínua em $x$ temos que $\lim _{h \rightarrow 0} \Delta f=0$ e por ser $G$ contínua $\frac{G(h)}{\|h\|}$ é limitada por $\|G\|$.

iii) Como,

$$
\Delta\left(\frac{1}{f}\right)+\frac{1}{f^{2}(x)} f^{\prime}(x)(h)=\frac{f^{2}(x)-f(x+h) f(x)+f(x+h) f^{\prime}(x)(h)}{f(x+h) f^{2}(x)},
$$


segue

$$
\begin{aligned}
\Delta\left(\frac{1}{f}\right)+\frac{F(h)}{f^{2}(x)} & =\frac{f^{2}(x)-f(x+h) f(x)+f(x+h) F(h)+f(x) F(h)-f(x) F(h)}{f(x+h) f^{2}(x)} \\
& =\frac{F(h) \Delta f-f(x)[\Delta f-F(h)]}{f(x+h) f^{2}(x)} .
\end{aligned}
$$

Portanto

$$
\begin{aligned}
\lim _{h \rightarrow 0} & \frac{\left\|\Delta\left(\frac{1}{f}\right)+\frac{F(h)}{f^{2}(x)}\right\|}{\|h\|}=\lim _{h \rightarrow 0} \frac{\|F(h) \Delta f-f(x)[\Delta f-F(h)]\|}{\left\|f(x+h) f^{2}(x) h\right\|} \\
& \leq \lim _{h \rightarrow 0} \frac{1}{\left\|f(x+h) f^{2}(x)\right\|}\|\Delta f\| \frac{\|F(h)\|}{\|h\|}+\lim _{h \rightarrow 0} \frac{1}{\|f(x+h) f(x)\|} \frac{\|\Delta f-F(h)\|}{\|h\|} \\
& \leq \frac{1}{\left\|f(x)^{3}\right\|} \cdot 0 \cdot\|F\|+\frac{1}{\left\|f(x)^{2}\right\|} \cdot 0=0
\end{aligned}
$$

Isto termina a demonstração do teorema.

Definição 2.5 Sejam $R$ um anel e $R\left[x_{1}, \ldots, x_{r}\right]$ o anel dos polinômios em $r$ indeterminadas sobre $R$. O grau de um monômio ax $x_{1}^{k_{1}} \ldots x_{r}^{k_{r}}$ é o inteiro positivo $k_{1}+\ldots+k_{r}$. Um polinômio, $p\left(x_{1}, \ldots, x_{r}\right)$ no anel é chamado homogêneo se todos os seus termos, têm o mesmo grau.

Seja $V$ um $\mathbb{F}$-espaço vetorial de dimensão $n$ e $p\left(x_{1}, \ldots, x_{n}\right) \in \mathbb{F}\left[x_{1}, \ldots, x_{n}\right]$. O polinômio $p\left(x_{1}, \ldots, x_{n}\right)$ determina uma função polinomial $f: V \rightarrow \mathbb{F}$ dada por

$$
x=\sum_{i=1}^{n} \alpha_{i} v_{i} \mapsto p\left(\alpha_{1}, \ldots, \alpha_{n}\right),
$$

onde $\left\{v_{1}, \ldots, v_{n}\right\}$ é uma base fixa de $V$. Se o corpo for infinito, esta correspondência é injetora e portanto podemos identificar cada polinômio com sua correspondente função polinomial. Se $p\left(x_{1}, \ldots, x_{n}\right)$ é um polinômio homogêneo de grau $q$, a correspondente função polinomial é chamada uma forma de grau q. Assim por exemplo, dado que um polinômio homogêneo de grau 1 em $n$ indeterminadas tem a forma $p\left(x_{1}, \ldots, x_{n}\right)=\sum_{i=1}^{n} c_{i} x_{i}, c_{i} \in \mathbb{F}$, então $f\left(\sum_{i=1}^{n} \alpha_{i} v_{i}\right)=p\left(\alpha_{1}, \ldots, \alpha_{n}\right)=\sum_{i=1}^{n} c_{i} \alpha_{i}$ que é um funcional linear ou 1-forma sobre $V$. Se $p\left(x_{1}, \ldots, x_{n}\right)$ é um polinômio homogêneo de grau 2 , então $p\left(x_{1}, \ldots, x_{n}\right)=\sum_{i \leq j} c_{i j} x_{i} x_{j}$, onde $c_{i j} \in \mathbb{F}$; portanto a correspondente forma de grau 2 é uma forma quadrática $Q$, dada por $x=\sum_{i=1}^{n} \alpha_{i} v_{i} \mapsto \sum_{i<j} c_{i j} \alpha_{i} \alpha_{j}$. Observe que para $\lambda \in \mathbb{F}, Q(\lambda x)=\lambda^{2} Q(x)$ e também sua forma polar $B: V \times V \rightarrow \mathbb{F}$ dada por $B(x, y)=Q(x+y)-Q(x)-Q(y)$ é uma forma bilinear que satisfaz $Q(x)=\frac{1}{2} B(x, x)$. Podemos definir também função polinomial homogênea da seguinte forma.

Definição 2.6 Seja $X$ um $\mathbb{F}$-espaço vetorial. Então $f: X \rightarrow \mathbb{F}$ é uma função polinomial homogênea de grau $n \geq 0$, se existir uma função n-linear $g: \underbrace{X \times \ldots \times X}_{n-\text { vezes }} \rightarrow \mathbb{F}$ tal que $f(x)=g(x, \ldots, x)$ para todo $x \in X$. Uma soma de funções polinomiais homogêneas é uma função polinomial. 
Por exemplo se $\varphi_{1}$ e $\varphi_{2}$ são funcionais lineares sobre uma $\mathbb{F}$-álgebra $A$, seja $f: A \rightarrow \mathbb{F}$, dada por $f(x)=\operatorname{det}\left[\varphi_{i}\left(x^{j}\right)\right], 1 \leq i, j \leq 2$,

$$
f(x)=\varphi_{1}(x) \varphi_{2}\left(x^{2}\right)-\varphi_{2}(x) \varphi_{1}\left(x^{2}\right) .
$$

Então, $f$ é uma função polinomial, pois se, $g: A \times A \times A \rightarrow \mathbb{F}$, é dada por, $g(x, y, z)=$ $\varphi_{1}(x) \varphi_{2}(y z)$, obtemos que $g$ é 3-linear e $g(x, x, x)=\varphi_{1}(x) \varphi_{2}\left(x^{2}\right)$. De igual forma se $g_{1}$ : $A \times A \times A \rightarrow \mathbb{F}$, é definida por $g_{1}(x, y, z)=\varphi_{2}(x) \varphi_{1}(y z)$, segue que $g_{1}$ é 3-linear e $g_{1}(x, x, x)=$ $\varphi_{2}(x) \varphi_{1}\left(x^{2}\right)$. Assim, $f(x)=g(x)-g_{1}(x)$ é uma soma de funções polinomiais homogêneas, nesse caso de grau 3.

Seja $X=\mathbb{R}^{m}, m \geq 1$ e $f: \mathbb{R}^{m} \rightarrow \mathbb{R}$ uma função polinomial homogênea de grau dois (não nula), então existe uma aplicação bilinear $g: \mathbb{R}^{m} \times \mathbb{R}^{m} \rightarrow \mathbb{R}$, tal que $f(x)=g(x, x)$ para todo $x$. É fácil provar que o conjunto $Z=\left\{x \in \mathbb{R}^{m} \mid f(x) \neq 0\right\}$ é denso em $\mathbb{R}^{m}$. Suponha o contrário. Existe $x_{0} \in \mathbb{R}^{m}$ e $\varepsilon>0$ tal que para todo $x \in \mathbb{R}^{m}$, com $\left\|x_{0}-x\right\|<\varepsilon$, tem-se $f(x)=0$. Seja $x_{1} \in \mathbb{R}^{m}$ tal que $f\left(x_{1}\right)=g\left(x_{1}, x_{1}\right) \neq 0$. Definamos $h: \mathbb{R} \rightarrow \mathbb{R}$, por

$$
h(\lambda)=f\left(x_{\lambda}\right)=g\left(x_{\lambda}, x_{\lambda}\right),
$$

onde $x_{\lambda}:=x_{0}+\lambda\left(x_{1}-x_{0}\right)$. Usando que $g$ é bilinear, temos que

$$
h(\lambda)=\alpha \lambda+\sigma \lambda^{2}, \quad(\lambda \in \mathbb{R}),
$$

onde $\alpha=g\left(x_{1}, x_{0}\right)+g\left(x_{0}, x_{1}\right)$ e $\sigma=g\left(x_{1}-x_{0}, x_{1}\right)-g\left(x_{1}-x_{0}, x_{0}\right)$ são constantes. Se $|\lambda|<\frac{\varepsilon}{\|t-y\|}$, então $\left\|x_{\lambda}-x_{0}\right\|<\varepsilon \operatorname{logo} h(\lambda)=f\left(x_{\lambda}\right)=0$. Como um polinômio de grau 2 em uma variável, tem no máximo duas raízes, temos que $h$ é identicamente zero, em contradição com o fato de que $h(1)=f\left(x_{1}\right) \neq 0$.

Temos o seguinte resultado geral.

Lema 2.1 Sejam $X$ um espaço normado sobre $\mathbb{F}$ e $f: X \rightarrow \mathbb{F}$ uma função polinomial não nula. Então $Z=\{x \in X \mid f(x) \neq 0\}$ é denso em $X$.

\section{Demonstração:}

Temos que $f=\sum_{i=1}^{p} f_{i}$, onde cada $f_{i}$ é uma função polinomial homogênea de grau $n_{i}$. Falamos que $n=\max \left\{n_{1}, \ldots, n_{p}\right\}$ é o grau de $f$.

Suponhamos que existe $x_{0} \in X$ e $\varepsilon>0$ tal que se $\left\|x-x_{0}\right\|<\varepsilon$ então $f(x)=0$. Seja $x_{1} \in X$ tal que $f\left(x_{1}\right) \neq 0$ e definimos $h: \mathbb{F} \rightarrow \mathbb{F}$ por $h(\lambda)=f\left(x_{\lambda}\right)$ onde $\left.x_{\lambda}=x_{0}+\lambda\left(x_{1}-x_{0}\right)\right)$. Temos que $h(1)=f\left(x_{1}\right) \neq 0$. Observamos que $h$ determina uma função polinomial na variável $\lambda$ de grau $n$, mas quando $|\lambda|<\frac{\varepsilon}{\left\|x_{1}-x_{0}\right\|}$, temos que $\left\|x_{\lambda}-x_{0}\right\|<\varepsilon$, portanto, $h(\lambda)=0$ o que é absurdo, pois $h$ tem no máximo $n$ raízes.

Seja agora $A$ uma $\mathbb{R}$-álgebra absolutamente valuada algébrica. Por ser $A$ algébrica, para cada $a \in A \backslash\{0\}$,temos que $A(a)$ é uma $\mathbb{R}$-álgebra absolutamente valuada de dimensão finita e por Teorema 1.6, sabemos que $\operatorname{dim}(A(a))$ é $1,2,4$, ou 8. O número $m(a)$ representará o máximo natural tal que o conjunto $\left\{a^{1}, \ldots, a^{m(a)}\right\}$ é linearmente independente em $A(a)$, onde $a^{i+1}=a^{i} a$. Também definimos $m(A):=\max \{m(a) \mid a \in A \backslash\{0\}\}$.

Lema 2.2 Seja $\Omega=\{a \in A \backslash\{0\} \mid m(a)=m(A)\}$. Então $\Omega$ é denso e aberto em $A$.

\section{Demonstração:}

Seja $m:=m(A)$. Dado $a \in \Omega$, temos que o conjunto $\left\{a^{1}, \ldots, a^{m}\right\}$ é linearmente independente. Sejam $B$ o subespaço $m$-dimensional de $A$ gerado por esse conjunto e $\left\{f_{1}, \ldots, f_{m}\right\}$ a base dual 
de $B$, isto é, $f_{i}: B \rightarrow \mathbb{R}$ é linear e $f_{i}\left(a^{j}\right)=\delta_{i j}, 1 \leq i, j \leq m$. Como cada $f_{i}$ é contínuo sobre $B$, aplicamos o teorema de Hahn-Banach, Teorema 2.4, e obtemos um conjunto $\left\{\varphi_{1}, \ldots, \varphi_{m}\right\}$ de funcionais lineares limitados sobre $A$ tal que $\varphi_{i}$ é uma extensão de $f_{i}$ para $i=1, \ldots, m$. Portanto $\varphi_{i}\left(a^{j}\right)=\delta_{i j}$ para $1 \leq i, j \leq m$. Para cada $x \in A$, consideramos a matriz $\left[\varphi_{i}\left(x^{j}\right)\right] \in$ $M_{m}(\mathbb{R})$. Definimos $f: A \rightarrow \mathbb{R}$, por

$$
f(x)=\operatorname{det}\left[\varphi_{i}\left(x^{j}\right)\right]=\sum_{\sigma \in S_{m}} s g(\sigma) \varphi_{1}\left(x^{\sigma(1)}\right) \ldots \varphi_{m}\left(x^{\sigma(m)}\right) .
$$

Onde $S_{m}$ é o grupo simétrico de ordem $m$ e $s g(\sigma)$ é o sinal da permutação $\sigma$. Temos que $f$ é uma função polinomial Fréchet diferenciável pois cada somando é um produto de formas lineares limitadas, as $\varphi_{i}$, Fréchet diferenciáveis. Seja $\Omega_{a}=\{x \in A \mid f(x) \neq 0\}$. Pela definição de $\Omega$, se $x \in A-\Omega$, então o conjunto $\left\{x^{1}, \ldots, x^{m}\right\}$ é linearmente dependente e, daí, a família $\left\{\left(\varphi_{1}\left(x^{1}\right), \ldots, \varphi_{m}\left(x^{1}\right)\right), \ldots,\left(\varphi_{1}\left(x^{m}\right), \ldots, \varphi_{m}\left(x^{m}\right)\right)\right\}$ de vetores em $\mathbb{F}^{m}$ é linearmente dependente $\operatorname{logo} f(x)=0$. Assim, $\Omega_{a} \subseteq \Omega$. Por outro lado, por Lema 2.1, $\Omega_{a}$ é denso em $A$ e como $\Omega_{a} \subseteq \Omega$ obtemos que $\Omega$ é denso em $A$.

Se $\Omega_{a}$ não for aberto existiria $y \in \Omega_{a}$ tal que para todo $\varepsilon>0, B(y, \varepsilon) \cap\left(A \backslash \Omega_{a}\right) \neq \emptyset$. Para cada $n$ inteiro positivo seja $x_{n} \in B(y, 1 / n) \cap\left(A \backslash \Omega_{a}\right)$. A sequência $\left\{x_{n}\right\}_{n \in \mathbb{N}}$, converge para $y$. Dado que $f\left(x_{n}\right)=0$ para todo $n$ e $f$ é contínua obtemos que $f(y)=\lim _{n \rightarrow \infty} f\left(x_{n}\right)=0$, que não é possível pois $y \in \Omega_{a}$. Portanto $\Omega_{a}$ é aberto.

Finalmente observe que $f(a)=1$, o que implica que $a \in \Omega_{a}$. Assim, provamos que para cada $a \in \Omega$ existe um aberto $\Omega_{a}$ que contém $a$ e está contido em $\Omega$. Consequentemente, $\Omega$ é aberto.

Lema 2.3 Seja A uma $\mathbb{R}$-álgebra absolutamente valuada algébrica. Para a $\in A \backslash\{0\}$, seja $\left(\alpha_{1}, \ldots, \alpha_{m(a)}\right)$ a única $m(a)$-upla em $\mathbb{R}^{m(a)}$ tal que $a^{m(a)+1}=\alpha_{1} a^{1}+\ldots+\alpha_{m(a)} a^{m(a)}$. Então $\alpha_{1}=\|a\|^{m(a)}$ ou $\alpha_{1}=-\|a\|^{m(a)}$.

\section{Demonstração:}

Seja $R_{a}: A(a) \rightarrow A(a)$, dada por $b \mapsto b a$. Esta aplicação é linear e seja $p(x) \in \mathbb{R}[x]$, o polinômio minimal de $R_{a}$. Temos, pelo Lema 1.9 , que toda raiz de $R_{a}$ tem módulo $\|a\|$. Como $R_{a}$ é uma transformação linear, $A(a)$ tem estrutura de $\mathbb{R}[x]$-módulo e

$$
\operatorname{ann}(a)=\left\{q(x) \in \mathbb{R}[x] \mid q\left(R_{a}\right)(a)=0\right\}
$$

é um ideal de $\mathbb{R}[x]$. Seja $s(x)=x^{m(a)}-\alpha_{m(a)} x^{m(a)-1}-\ldots-\alpha_{2} x-\alpha_{1}$ um polinômio mônico de grau $m(a)$ em $\mathbb{R}[x]$ que satisfaz $s\left(R_{a}\right)(a)=0$. Temos que $s(x) \in \operatorname{ann}(a)$ e pela definição de $m(a), s(x)$ é o polinômio de menor grau tal que $s\left(R_{a}\right)(a)=0$, isto é, $s(x)$ gera ann $(a)$. Como $p(x)$ é o polinômio minimal de $R_{a}, p(x) \in \operatorname{ann}(a)$, então $s(x)$ divide a $p(x)$ e assim toda raiz complexa de $s(x)$ tem módulo $\|a\|$. Agora, $s(x)$ visto como polinômio sobre $\mathbb{C}$ é tal que $s(x)=\left(x-\lambda_{1}\right)\left(x-\lambda_{2}\right) \ldots\left(x-\lambda_{m(a)}\right)$, então $\left|\alpha_{1}\right|=\left|\lambda_{1} \ldots \lambda_{m(a)}\right|=\|a\| \ldots\|a\|, m(a)$-vezes, $\left|\alpha_{1}\right|=\|a\|^{m(a)}$. Portanto, $\alpha_{1}=\|a\|^{m(a)}$ ou $\alpha_{1}=-\|a\|^{m(a)}$.

Lembramos que se $M \in M_{n}(\mathbb{R})$ é invertível, $X=\left(x_{1}, \ldots, x_{n}\right)^{t}$ é o vetor coluna das incógnitas e $B=\left(b_{1}, \ldots, b_{n}\right)^{t}$ é um elemento de $\mathbb{R}^{n}$, então o sistema linear $M X=B$ tem solução única, com $x_{i}=\frac{\operatorname{det} M_{i}}{\operatorname{det} M}$ (Regra de Cramer). Sendo $M_{i} \in M_{n}(\mathbb{R})$ a matriz obtida substituindo a $i$-ésima coluna de $M$ por $B$.

Teorema 2.15 Seja A uma $\mathbb{R}$-álgebra absolutamente valuada algébrica. Então a norma $\|\|:. A \rightarrow \mathbb{R}$ é Fréchet diferenciável em cada ponto de $\Omega=\{x \in A \backslash\{0\} \mid m(x)=m(A)\}$. 


\section{Demonstração:}

Seja $a \in \Omega$ fixo e por simplicidade denotemos por $m$ o inteiro positivo $m(a)=m(A)$. Consideremos o conjunto aberto $\Omega_{a}$ e a família de formas lineares limitadas $\Phi=\left\{\varphi_{1}, \ldots, \varphi_{m}\right\}$ definidas no Lema 2.2 em relação ao elemento $a$. Para cada $x \in \Omega_{a}$ o conjunto $\left\{x^{1}, \ldots, x^{m}\right\}$ é linearmente independente em $A(x)$, logo existe um único $\left(\lambda_{1}(x), \ldots, \lambda_{m}(x)\right) \mathrm{em} \mathbb{R}^{m}$ tal que $x^{m+1}=\lambda_{1}(x) x^{1}+\lambda_{2}(x) x^{2}+\ldots+\lambda_{m}(x) x^{m}$. Por outro lado, a família $\Phi$ nos fornece o seguinte sistema linear de equações

$$
\left\{\begin{array}{c}
\sum_{i=1}^{m} \lambda_{i}(x) \varphi_{1}\left(x^{i}\right)=\varphi_{1}\left(x^{m+1}\right) \\
\vdots \\
\sum_{i=1}^{m} \lambda_{i}(x) \varphi_{m}\left(x^{i}\right)=\varphi_{m}\left(x^{m+1}\right)
\end{array}\right.
$$

Pela regra de Cramer temos que $\lambda_{i}(x)=\frac{\operatorname{det}\left(M_{i}\right)}{\operatorname{det}\left(\varphi_{i}\left(x^{j}\right)\right)}$, onde $M_{i}$ é obtida substituindo na matriz $\left(\varphi_{i}\left(x^{j}\right)\right)$ a $i$-ésima coluna por $\left(\varphi_{1}\left(x^{m+1}\right), \ldots, \varphi_{m}\left(x^{m+1}\right)\right)^{t}$. Como $\lambda_{1}(x)$ é o quociente de duas funções polinomiais com $\operatorname{det}\left(\varphi_{i}\left(x^{j}\right) \neq 0\right)$, temos que

$$
\lambda_{1}: \Omega_{a} \rightarrow \mathbb{R}, \quad \lambda_{1}(x)=\frac{\operatorname{det}\left(M_{1}\right)}{\operatorname{det}\left(\varphi_{i}\left(x^{j}\right)\right)},
$$

é Fréchet diferenciável em $a$. Pelo Lema 2.3, $\lambda_{1}(x)= \pm\|x\|^{m}$ e dado que $\lambda_{1}$ é contínua, $\lambda_{1}$ tem o mesmo sinal em qualquer conjunto aberto conexo de $A$ contendo a $a$ e contido em $\Omega_{a}$. Assim, $\|x\|^{m}$ é Fréchet diferenciável em $a$. Existe $T \in B L(A, \mathbb{R})$ tal que

$$
\lim _{h \rightarrow 0} \frac{\left|\lambda_{1}(a+h)-\lambda_{1}(a)-T(h)\right|}{\|h\|}=0=\lim _{h \rightarrow 0} \frac{\left|\|(a+h)\|^{m}-\|(x)\|^{m}-T(h)\right|}{\|h\|} .
$$

Sabemos, pela regra do produto para derivadas, que se a função $\|\cdot\|$ for Fréchet diferenciável em $a$, então sua derivada é $\alpha^{-1} T$, onde $\alpha=m\|a\|^{m-1}$.

Por outro lado temos que

$$
\|a+h\|^{m}-\|a\|^{m}=(\|a+h\|-\|a\|) g(h), \quad \text { com } \quad g(h)=\sum_{i=0}^{m-1}\|a+h\|^{i}\|a\|^{m-i-1} .
$$

Por ser a norma contínua, o limite $\lim _{h \rightarrow 0} g(h)$ existe e é igual a $\alpha$. Agora

$$
\begin{aligned}
& \lim _{h \rightarrow 0} \frac{|\| a+h||-||a|\left|-\frac{1}{\alpha} T(h)\right|}{\|h\| \mid}=\lim _{h \rightarrow 0} \frac{1}{\alpha} \frac{|(|| a+h \|-|| a||) \alpha-T(h)|}{\|h\|} \\
& \quad=\frac{1}{\alpha} \lim _{h \rightarrow 0} \frac{|(\|a+h\|-\|a\|)(g(h)-g(h)+\alpha)-T(h)|}{\|h\|} \\
& \quad \leq \frac{1}{\alpha} \lim _{h \rightarrow 0} \frac{|(\|a+h\|-\|a\|) g(h)-T(h)|}{\|h\|}+\frac{1}{\alpha} \lim _{h \rightarrow 0}|(|| a+h\|-\| a \|)(-g(h)+\alpha)| \\
& \quad=\frac{1}{\alpha} \lim _{h \rightarrow 0} \frac{\left|\|a+h\|^{m}-\|a\|^{m}-T(h)\right|}{\|h\|}+0=0+0=0 .
\end{aligned}
$$

Portanto, a norma é Fréchet diferenciável em $a$.

A seguinte é uma definição dada em [9].

Definição 2.7 Seja $X$ um espaço normado sobre $\mathbb{F}$. Dizemos que $X$ é suave em $x \in X$, $x \neq 0$, se existir um único funcional linear $f$ em $X^{\prime}$ de norma 1 tal que $f(x)=\|x\|$. 
Observe que o teorema de Hahn-Banach garante a existência de um tal funcional. Por [21] página 487 observamos que a suavidade de $X$ em $x$ é equivalente com o fato de que a norma de $X$ é Gateaux diferenciável em $x$.

Proposição 2.3 Sejam $X$ um espaço normado sobre $\mathbb{F}, T: X \rightarrow X$ uma aplicação linear tal que $\|T\| \leq 1$ e $M$ um subespaço de $X$, de dimensão finita tal que a restrição da norma de $X$ a $M$ está induzida por um produto interno. Assumimos que $T(x)=x$ para todo $x \in M$ $e$ que $X$ é suave em todo elemento de norma 1 de $M$. Então existe uma projeção linear continua $\pi: X \rightarrow X$ tal que $\pi(X)=M$ e ker $(\pi)$ é T-invariante.

\section{Demonstração:}

Seja $\langle\cdot, \cdot\rangle$ o produto interno em $M$ tal que $\|x\|^{2}=\langle x, x\rangle$ para todo $x \in M$, e seja $\left\{e_{1}, \ldots, e_{k}\right\}$ base ortonormal de $M$. Pelo Corolário 2.2, existem funcionais $\varphi_{1}, \ldots, \varphi_{k}$ contínuos de norma 1 sobre $X$ tais que $\varphi_{i}(x)=\left\langle x, e_{i}\right\rangle$ para todo $x \in M$ e $1 \leq i \leq k$. Definimos $\pi: X \rightarrow X$ por $\pi(x)=\sum_{i=1}^{k} \varphi_{i}(x) e_{i}$. O operador $\pi$ é linear contínuo e $\pi(X)=M$. Como $\pi\left(e_{i}\right)=e_{i}$ para todo $e_{i}$ na base, obtemos que $\pi^{2}=\pi$. Assim, $\pi$ é projeção.

Temos que $\left\|\varphi_{i}\right\|=1,1 \leq i \leq k$, e $\varphi_{i}\left(e_{i}\right)=1=\left\|e_{i}\right\|$. Definimos o seguinte funcional linear contínuo $\psi_{i}=\varphi_{i} \circ T$. Como $T(x)=x$ para todo $x \in M$, obtemos que $\psi_{i}\left(e_{i}\right)=e_{i} \operatorname{logo}$ $\left\|\psi_{i}\right\| \geq 1$. Por outro lado, para cada $x \in X$,

$$
\left\|\psi_{i}(x)\right\|=\left\|\varphi_{i}(T(x))\right\| \leq\left\|\varphi_{i}\right\|\|T\|\|x\|=\|T\|\|x\| \leq\|x\|,
$$

$\operatorname{logo}\left\|\psi_{i}\right\| \leq 1$. Uma vez que a outra desigualdade foi provada acima, temos que $\left\|\psi_{i}\right\|=1$. Como $X$ é suave em todo elemento de norma um de $M$, obtemos que $\psi_{i}=\varphi_{i}$ para todo $i$.

Seja $x \in \operatorname{ker}\left(\varphi_{i}\right)$. Então $0=\varphi_{i}(x)=\psi_{i}(x)=\varphi_{i}(T(x))$, assim $T(x) \in \operatorname{ker}\left(\varphi_{i}\right)$ portanto $\operatorname{ker}\left(\varphi_{i}\right)$ é $T$-invariante, para todo $i, 1 \leq i \leq k$. Se $x \in k e r(\pi)$, então $0=\pi(x)=\sum_{i=1}^{k} \varphi_{i}(x) e_{i}$, e como $\left\{e_{1}, \ldots, e_{k}\right\}$ é um conjunto linearmente independente, $\varphi_{i}(x)=0$ para todo $i$, logo $x \in \cap_{i=1}^{k} \operatorname{ker}\left(\varphi_{i}\right)$. Assim, $\operatorname{ker}(\pi)$ é $T$-invariante.

Proposição 2.4 Sejam $A$ uma $\mathbb{F}$-álgebra absolutamente valuada algébrica e a $\in A$ tal que $\|a\|=1$. Se A é suave em a, então é suave em todo elemento de norma 1 de $A(a)$.

\section{Demonstração:}

Temos que $A(a)$ é de dimensão finita e absolutamente valuada, portanto de divisão. Seja $b \in A(a)$ com $\|b\|=1$, então existe $c \in A(a)$ tal que $c b=a$. Como $A(a)$ é absolutamente valuada, $1=\|a\|=\|c b\|=\|c\|\|b\|=\|c\|$, então $L_{c}: A \rightarrow A$ é uma isometria linear. Suponhamos que $A$ é suave em $a$ e que não o é em $b$. Sejam $\psi, \varphi$ funcionais lineares de norma 1 em $A$ com $\psi \neq \varphi, \psi(b)=\|b\|=\varphi(b)$. A aplicação $L_{c}: A \rightarrow c A$ é sobrejetora e $L_{c}^{-1}: c A \rightarrow A$ também é uma isometria, então $\psi \circ L_{c}^{-1}: c A \rightarrow \mathbb{F}$ é linear e contínua,

$$
\psi\left(L_{c}^{-1}(a)\right)=\psi\left(L_{c}^{-1}(c b)\right)=\psi(b)=\|b\|=\|a\|
$$

e $\left\|\psi\left(L_{c}^{-1}(x)\right)\right\| \leq\left\|\psi \circ L_{c}^{-1}\right\|\|x\| \leq\|\psi\|\left\|L_{c}^{-1}\right\|\|x\|=\|x\|$ para todo $x \in A$. Portanto,

$$
\left\|\psi \circ L_{c}^{-1}\right\|=1 \quad \text { e } \quad \psi\left(L_{c}^{-1}(a)\right)=\|a\| .
$$

De forma análoga, podemos provar que $\left\|\varphi \circ L_{c}^{-1}\right\|=1$ e $\varphi\left(L_{c}^{-1}(a)\right)=\|a\|$. Como $\psi \neq \varphi$, obtemos que $\psi \circ L_{c}^{-1} \neq \varphi \circ L_{c}^{-1}$. O que mostra que $c A$ não é suave em $a$ por Teorema de Hahn-Banach, $A$ não é suave em $a$ o que é uma contradição. Consequentemente $A$ deve ser 
suave em $b$.

A seguinte definição de álgebra de é dada em [9].

Definição 2.8 Seja $A$ uma $\mathbb{F}$-álgebra algébrica. Se existir um número natural $m$ tal que $\operatorname{dim}(A(x)) \leq m$ para todo $x \in A$, então $A$ é chamada de grau limitado e, o menor número natural $m$ tal que $\operatorname{dim}(A(x)) \leq m$ para todo $x$ em $A$, é chamado o grau de $A$ e denotado por $\operatorname{deg}(A)=m$.

Observe que se $A$ é uma $\mathbb{R}$-álgebra absolutamente valuada algébrica então, pelo Teorema 1.6, $A$ é de grau limitado $\operatorname{com} \operatorname{deg}(A)=1,2,4$ ou 8 .

Proposição 2.5 Seja A uma $\mathbb{F}$-álgebra algébrica absolutamente valuada. Se $\operatorname{dim}(A(a))=$ $\operatorname{deg}(A)$ e se $b \in A \backslash\{0\}$ com $a b=b$, então $A(b)=A(a)$.

\section{Demonstração:}

Sabemos que $A(b)$ é absolutamente valuada e de dimensão finita, logo uma álgebra de divisão. Assim, existe $c \in A(b)$ tal que $c b=b$. Por outro lado, temos que $a b=b$, por isso $(a-c) b=0$ o que implica que $a=c$. Portanto $a \in A(b)$ donde $A(a) \subseteq A(b)$. Usando que $\operatorname{dim}(A(a))=$ $\operatorname{deg}(A)$, deve ser $\operatorname{dim}(A(b)) \leq \operatorname{dim}(A(a))$. Portanto, $A(b)=A(a)$. 


\section{Capítulo 3}

\section{Álgebras Algébricas Absolutamente Valuadas}

O objetivo da seguinte seção é expor alguns resultados sobre ultrafiltros e ultraprodutos, sendo o resultado principal a prova que o ultraproduto (de espaços normados) de uma família de $\mathbb{R}$-álgebras algébricas e absolutamente valuadas é uma $\mathbb{R}$-álgebra algébrica e absolutamente valuada, Teorema 3.1. O assunto da seguinte seção segue as linhas de [9], [11] e [23]. Aqui I denotará um conjunto qualquer não vazio.

\subsection{Ultrafiltros e Ultraprodutos}

Definição 3.1 Seja $\mathcal{P}(I)$, o conjunto dos subconjuntos de um conjunto nãa vazio $I$. Um subconjunto não vazio $\mathcal{F}$ de $\mathcal{P}(I)$ chama-se filtro sobre $I$ se satisfaz:

i) Fechado para interseções: Se $a, b \in \mathcal{F}$ então $a \cap b \in \mathcal{F}$,

ii) Fechado para extensões: Se $a \in \mathcal{F}$ e $a \subseteq c$ então $c \in \mathcal{F}$,

iii) Próprio: $\emptyset \notin \mathcal{F}$.

Observemos que por i), $\mathcal{F}$ é fechado para interseções finitas e, por ii), se $a \in \mathcal{F}$ e $b \in \mathcal{P}(I)$ então $a \cup b \in \mathcal{F}$ o que implica que $I \in \mathcal{F}$. Por iii) temos que $\mathcal{F} \neq \mathcal{P}(I)$.

O menor filtro que contem um dado elemento $a_{0} \in \mathcal{P}(I)$, chama-se principal e $a_{0}$ é um elemento principal do filtro. O filtro principal para $a_{0}$ é o conjunto $\left\{a \in \mathcal{P}(I): a_{0} \subset a\right\}$.

Um ultrafiltro, $\mathcal{U}$, sobre $I$ é um filtro maximal no sentido que não está propriamente contido em qualquer outro filtro sobre $I$.

Lema 3.1 Seja $\mathcal{U}$ um filtro em $\mathcal{P}(I)$. Então as seguintes condições são equivalentes:

i) $\mathcal{U}$ é ultrafiltro,

ii) Se $a \cup b \in \mathcal{U}$ então $a \in \mathcal{U}$ ou $b \in \mathcal{U}$,

iii) Se $a_{1} \cup \cdots \cup a_{n} \in \mathcal{U}$ então algum $a_{i} \in \mathcal{U}$,

iv) Dado $a \in \mathcal{P}(I), a \in \mathcal{U}$ ou $a^{\prime} \in \mathcal{U}$, onde $a^{\prime}$ é o complemento de a. 


\section{Demonstração:}

i) $\Rightarrow$ ii) Sejam $a \cup b \in \mathcal{U}, a \notin \mathcal{U}$ e $b \notin \mathcal{U}$. Mostremos que o seguinte conjunto,

$$
\mathcal{F}=\{d \in \mathcal{P}(I) \mid a \cap c \subseteq d \text { para algum } c \in \mathcal{U}\}
$$

é um filtro sobre $I$ que contém propriamente a $\mathcal{U}$. Se $c \in \mathcal{U}, a \cap c \subseteq c$ então $\mathcal{U} \subseteq \mathcal{F}$. Dado que $a=(a \cup b) \cap a$, seja $c=(a \cup b)$, então $a \in \mathcal{F}$. Assim, $\mathcal{U}$ está contido propriamente em $\mathcal{F}$. $\mathcal{F}$ é um filtro sobre $I$. De fato, se $x_{1}, x_{2}$ são elementos em $\mathcal{F}, a \cap c_{1} \subseteq x_{1}$ e $a \cap c_{2} \subseteq x_{2}$, para elementos $c_{1}, c_{2}$ em $\mathcal{U}$. Então, $a \cap\left(c_{1} \cap c_{2}\right) \subseteq x_{1} \cap x_{2}$ e como $\mathcal{U}$ é um filtro, $c_{1} \cap c_{2} \in \mathcal{U}$, daí $x_{1} \cap x_{2} \in \mathcal{F}$. Se $x \in \mathcal{F}$ e $y \in \mathcal{P}(I)$ tal que $x \subseteq y$ então pela definição de $\mathcal{F}, y \in \mathcal{F}$. Finalmente, se $\emptyset \in \mathcal{F}$ então existe $c \in \mathcal{U}$ tal que $c \cap a=\emptyset$, $\log 0 c \subseteq a^{\prime}$. Como $\mathcal{U}$ é um filtro, $a^{\prime} \in \mathcal{U}$, assim $a^{\prime} \cap b=a^{\prime} \cap(a \cup b)$, donde $a^{\prime} \cap b \in \mathcal{U}$. Como, $a^{\prime} \cap b \subseteq b, b \in \mathcal{U}$, o que é absurdo. Desse modo $\emptyset \notin \mathcal{F}$ e $\mathcal{F}$ é um filtro que contém propriamente a $\mathcal{U}$. O que não é possível.

É claro que ii) $\Leftrightarrow$ iii). Mostremos que ii) $\Rightarrow$ iv) Seja $a \in \mathcal{P}(I)$ e $a^{\prime}$ o complemento de $a$. Como $a \cup a^{\prime}=I \in \mathcal{U}$, obtemos que $a \in \mathcal{U}$ ou $a^{\prime} \in \mathcal{U}$.

iv) $\Rightarrow$ i) Se $\mathcal{U}$ está incluído propriamente em um filtro $\mathcal{F}$ sobre $I$, seja $d \in \mathcal{F}$ tal que $d \notin \mathcal{U}$, então $d^{\prime} \in \mathcal{U}$ obtemos assim que $\emptyset=d \cap d^{\prime} \in \mathcal{F}$ o que é absurdo pois $\mathcal{F}$ é próprio.

Lema 3.2 Todo filtro $\mathcal{F}$ sobre I está contido em um ultrafiltro $\mathcal{U}$.

\section{Demonstração:}

Seja $G=\{J \mid J$ é um filtro sobre $I$ e $\mathcal{F} \subseteq J\}$. $G$ é parcialmente ordenado pela inclusão. Seja $C$ uma cadeia de $G$, então a união dos elementos de $C$ é um filtro sobre $I$ e pelo Lema de Zorn existe um filtro maximal, $\mathcal{U}$, sobre $I$ tal que $\mathcal{F} \subseteq \mathcal{U}$.

Exemplo 3.1 i) Filtro principal: $\mathcal{F}\left(X_{0}\right)$ sobre $X$ determinado por um subconjunto $X_{0} \subset$ $X$, consistente de todos os subconjuntos $Y \supseteq X_{0}$ que contêm $X_{0}$.

ii) Ultrafiltro principal: $\mathcal{F}_{x_{0}}$ sobre $X$ determinado por um elemento $x_{0} \in X$ consistente de todos os subconjuntos $Y \subseteq X$ que contém $x_{0}$.

iii) Filtro cofinito ou de Fréchet: $\mathcal{F}_{\infty}$ sobre $X$ é o conjunto de todos os $A$ em $X$ tal que seu complemento é finito, isto é $\mathcal{F}_{\infty}=\{A \subseteq X: X \backslash A$ é finito $\}$. Para $X=\mathbb{N}$ uma base deste filtro é $\{[n, \infty): n \in \mathbb{N}\}$. $\mathcal{F}_{\infty}$ é um filtro mas não é um ultrafiltro. Pois se $B \in \mathbb{N}$ é o conjunto dos números pares, então $B \notin \mathcal{F}_{\infty}$ e também $B^{\prime} \notin \mathcal{F}_{\infty}$, contrário a iv) em Lema 3.1.

Sejam $I$ um conjunto infinito, $\mathcal{U}$ um ultrafiltro sobre $I$ e $X$ um espaço normado. Uma função $f: I \rightarrow X$ é dita convergente a $x \in X$ através de $\mathcal{U}$, ou $x$ é o $\mathcal{U}$-limite de $f$, denotado por

$$
\lim _{\mathcal{U}} f=x,
$$

se para cada $\varepsilon>0$ o conjunto, $\{i \in I \mid\|f(i)-x\|<\varepsilon\} \in \mathcal{U}$, isto é, se para todo aberto $V$ em $X$ com $x \in V$, temos que $f^{-1}(V) \in \mathcal{U}$. Podemos provar facilmente que se existir um limite $\lim _{\mathcal{U}} f$, ele é único.

Proposição 3.1 Sejam $f, g: I \rightarrow X$ funções tal que existem os limites $\lim _{\mathcal{U}} f=x e$ $\lim _{\mathcal{U}} g=y$. Então: 
i) Existe o limite de $f+g$ em $\mathcal{U}$ e $\lim _{\mathcal{U}}(f+g)=x+y$;

ii) Se $f$ for constante em algum $A \in \mathcal{U}$, isto é $f(i)=x_{0}$ para todo $i \in A$, então $x=x_{0}$;

iii) Se $X=\mathbb{R}$ e existir $A \in \mathcal{U}$ tal que $f(i) \leq g(i)$ para todo $i \in A$, então $x \leq y$;

iv) $S e X=\mathbb{R}$, então existe o limite de $f g$ em $\mathcal{U}$ e $\lim _{\mathcal{U}}(f g)=x y$;

\section{Demonstração:}

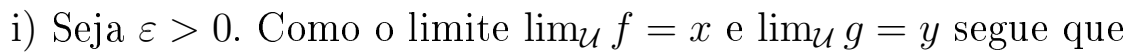

$$
A=\left\{i \in I \mid\|f(i)-x\|<\frac{\varepsilon}{2}\right\} \in \mathcal{U} \quad \text { e } \quad B=\left\{i \in I \mid\|g(i)-y\|<\frac{\varepsilon}{2}\right\} \in \mathcal{U} .
$$

Portanto, para todo $i \in A \cap B$, temos

$$
\|f(i)+g(i)-x-y\| \leq\|f(i)-x\|+\|g(i)-y\|<\varepsilon
$$

$\log 0$

$$
A \cap B \subseteq\{i \in I \mid\|(f+g)(i)-(x+y)\|<\varepsilon\} \in \mathcal{U} .
$$

Consequentemente, o $\mathcal{U}$-limite de $f+g$ existe e é $x+y$.

ii) Para cada $\varepsilon>0$, temos que $A \subseteq\left\{i \in I \mid\left\|f(i)-x_{0}\right\|<\varepsilon\right\} \in \mathcal{U}$. Logo, o $\mathcal{U}$-limite de $f$ existe e é $x_{0}$.

Provaremos iii) por redução ao absurdo. Suponhamos que $x>y$. Seja $0<\varepsilon<(x-y) / 2$. Então

$$
B:=\{i \in I:|f(i)-x|<\varepsilon\} \in \mathcal{U}, \quad C:=\{i \in I:|g(i)-y|<\varepsilon\} \in \mathcal{U} .
$$

Por ser $\mathcal{U}$ um ultrafiltro propário, $A \cap B \cap C \neq \emptyset$ e se $i \in A \cap B \cap C$, então

$$
g(i)<\frac{x+y}{2}<f(i)
$$

em contradição com a hipótese.

iv) Seja $\varepsilon>0$. Para

$$
0<\delta<\min \left\{\frac{1}{2}, \frac{\varepsilon}{2}, \frac{\varepsilon}{2(|x|+|y|+1)}\right\}
$$

temos que

$$
B:=\{i \in I:\|f(i)-x\|<\delta\} \in \mathcal{U}, \quad C:=\{i \in I:\|g(i)-y\|<\delta\} \in \mathcal{U} .
$$

Assim, $\emptyset \neq B \cap C \in \mathcal{U}$ e para cada $i \in B \cap C$, temos

$$
\begin{aligned}
|f(i) g(i)-x y| & =|f(i) g(i)-f(i) y+f(i) y-x y|=|f(i)(g(i)-y)+(f(i)-x) y| \\
& \leq|f(i)(g(i)-y)|+|(f(i)-x) y| \leq|f(i)||g(i)-y|+|f(i)-x||y| \\
& <|f(i)| \delta+\delta|y|=\delta(|f(i)|+|y|)<\delta(|f(i)-x+x|+|y|) \\
& <\delta(|f(i)-x|+|x|+|y|)<\delta(\delta+|x|+|y|)=\delta^{2}+\delta(|x|+|y|)<\varepsilon .
\end{aligned}
$$

Portanto

$$
B \cap C \subseteq\{i \in I:|(f g)(i)-x y|<\varepsilon\} \in \mathcal{U}
$$


Consequentemente, o $\mathcal{U}$-limite de $f g$ existe e é $x y$.

$X$ é dito $\mathcal{U}$-completo se para qualquer família $\left\{x_{i}\right\}_{i \in I}$ em $X$ existe $x \in X$ tal que $\lim _{\mathcal{U}} x_{i}=x$. E, $X$ é ultracompleto se é $\mathcal{U}$-completo para todo $I$ e todo ultrafiltro $\mathcal{U}$ sobre $I$.

Temos o seguinte resultado. ( Para mais informação ver [23], pag 5.)

Lema 3.3 Seja X um espaço normado. Então X é compacto se, e somente se, X é ultracompleto.

\section{Demonstração:}

Seja $\left\{x_{i}\right\}_{i \in I}$ em $X$ e $\mathcal{U}$ um ultrafiltro sobre $I$. Suponhamos que para cada $x \in X, \lim _{\mathcal{U}} x_{i} \neq x$. Então para cada $x \in X$ existe um aberto $V_{x}$ tal que $x \in V_{x}$ e $\left\{i \in I \mid x_{i} \in V_{x}\right\} \notin \mathcal{U}$ e daí, $Y_{x}=\left\{i \in I \mid x_{i} \notin V_{x}\right\} \in \mathcal{U}$. Como $X$ é compacto, existe um conjunto finito $B \subseteq X$ tal que a família $\left\{V_{x} \mid x \in B\right\}$ cobre a $X$. Então, o conjunto $Y=\cap_{x \in B} Y_{x} \in \mathcal{U}$ pois $\mathcal{U}$ é um filtro. Para $i \in Y$, obtemos que $x_{i} \notin \bigcup_{x \in X} V_{x}$, o que é absurdo.

Reciprocamente, suponhamos que $X$ é ultracompleto. Necessitamos provar que se $\Phi=$ $\left\{V_{i}\right\}_{i \in I}$ é uma coleção de subconjuntos abertos de $X$ tal que nenhuma subcoleção finita é uma cobertura de $X$ então a união dos $V_{i}$ não pode ser uma cobertura de $X$. Primeiro, como nenhuma subcoleção é uma cobertura de $X$ podemos construir um filtro gerado por seus complementos:

$$
\mathcal{F}=\left\{A \subseteq X: \bigcap_{j=1}^{n}\left(X \backslash V_{i_{j}}\right) \subseteq A \text { para um número finito de } V_{i_{1}}, \ldots, V_{i_{n}} \in \Phi\right\} .
$$

Podemos estender $\mathcal{F}$ até um ultrafiltro $\mathcal{U}$. Seja $f: X \rightarrow X$ a aplicação identidade. Por hipótese, existe $x \in X$ tal que $\lim _{\mathcal{U}} f=x$. Se algum $\operatorname{dos} V_{i}$ contém $x$, então $V_{i} \in \mathcal{U}$ por definição de convergência. Porém, nós também temos que $X \backslash V_{i} \in \mathcal{F} \subset \mathcal{U}$ e isto é uma contradição. Portanto $x$ está fora da união dos $V_{i}$, como queríamos provar.

No Lema 3.3 podemos considerar que $X$ é simplesmente um espaço topológico.

Consideremos agora $\left\{X_{i}\right\}_{i \in I}$, uma família de espaços normados com $I$ um conjunto infinito e $\mathcal{U}$ um ultrafiltro sobre $I$. Definimos agora o ultraproduto de espaços normados. Seja $l_{\infty}\left(I, X_{i}\right)=\left\{\left\{x_{i}\right\}_{i \in I} \in \prod_{i \in I} X_{i} \mid \sup \left\{\left\|x_{i}\right\| \mid i \in I\right\}<\infty\right\}$ e para $\left\{x_{i}\right\}_{i \in I} \in l_{\infty}\left(I, X_{i}\right)$ a aplicação $\left\|\left\{x_{i}\right\}_{i \in I}\right\|=\sup \left\{\left\|x_{i}\right\| \mid i \in I\right\}=\sup _{I}\left\|x_{i}\right\|$, é uma norma sobre $l_{\infty}\left(I, X_{i}\right)$.

Seja $\left\{x_{i}\right\}_{i \in I} \in l_{\infty}\left(I, X_{i}\right)$. A aplicação $f: I \rightarrow \mathbb{R}$ dada por $i \mapsto\left\|x_{i}\right\|$ é limitada, isto é, existe $M>0$ tal que $\|f(i)\| \leq M$ para todo $i \in I$. Como $[-M, M]$ é compacto em $\mathbb{R}$, obtemos por lema anterior que $f$ converge através do $\mathcal{U}$. Tomemos agora o seguinte conjunto

$$
N_{\mathcal{U}}=\left\{\left\{x_{i}\right\}_{i \in I} \in l_{\infty}\left(I, X_{i}\right) \mid \lim _{\mathcal{U}}\left\|x_{i}\right\|=0\right\} .
$$

O conjunto $N_{\mathcal{U}}$ é um subespaço vetorial de $l_{\infty}\left(I, X_{i}\right)$, na verdade um subespaço vetorial fechado. Sejam $\left\{x_{i}\right\}_{i \in I},\left\{y_{i}\right\}_{i \in I} \in N_{\mathcal{U}}$ e $0 \neq \lambda \in \mathbb{F}$. Dado $\varepsilon>0$ os conjuntos

$$
A=\left\{i \in I:\left\|x_{i}\right\|<\frac{\varepsilon}{2}\right\}, \quad B=\left\{i \in I:\left\|y_{i}\right\|<\frac{\varepsilon}{2|\lambda|}\right\}
$$

estão em $\mathcal{U}$. Se $i \in A \cap B$, então

$$
\left\|x_{i}+\lambda y_{i}\right\| \leq\left\|x_{i}\right\|+\left\|\lambda y_{i}\right\|=\left\|x_{i}\right\|+|\lambda||| y_{i} \|<\frac{\varepsilon}{2}+|\lambda| \frac{\varepsilon}{2|\lambda|}=\varepsilon .
$$

Assim, $A \cap B \subseteq\left\{i \in I:\left\|x_{i}+\lambda y_{i}\right\|<\varepsilon\right\} \in \mathcal{U}$. Isto prova que $N_{\mathcal{U}}$ é um subespaço vetorial. Seja agora $a=\left\{x_{i}\right\}_{i \in I} \in \overline{N_{\mathcal{U}}}$. Então existe uma sequência $\left\{b_{n}\right\}_{n \in \mathbb{N}}$ em $N_{\mathcal{U}}$ tal que 
$\lim _{n \rightarrow \infty} b_{n}=a$. Assim, se $b_{n}=\left\{y_{i}^{n}\right\}_{i \in I}$, então $\lim _{\mathcal{U}}\left\|y_{i}^{n}\right\|=0$ e dado $\varepsilon>0$ existe $n_{0} \in \mathbb{N}$ tal que se $n \geq n_{0},\left\|b_{n}-a\right\|<\frac{\varepsilon}{2}$ onde $\left\|b_{n}-a\right\|=\sup _{i \in I}\left\|y_{i}^{n}-x_{i}\right\|$. Portanto, $\left\|y_{i}^{n}-x_{i}\right\|<\frac{\varepsilon}{2}$ para todo $i \in I$, sempre que $n \geq n_{0}$, em particular $\left\|y_{i}^{n_{0}}-x_{i}\right\|<\frac{\varepsilon}{2}$. Como $\left\{y_{i}^{n_{0}}\right\}_{i \in I} \in N_{\mathcal{U}} \mathrm{O}$ conjunto $Z=\left\{i \in I \mid\left\|y_{i}^{n_{0}}\right\|<\frac{\varepsilon}{2}\right\} \in \mathcal{U}$. Pela desigualdade triangular temos que

$$
\left\|x_{i}\right\|=\left\|x_{i}-y_{i}^{n_{0}}+y_{i}^{n_{0}}\right\| \leq\left\|x_{i}-y_{i}^{n_{0}}\right\|+\left\|y_{i}^{n_{0}}\right\|,
$$

então $\left\|x_{i}\right\|<\varepsilon$ para todo $i \in Z$. Assim, $Z \subseteq\left\{i \in I \mid\left\|x_{i}\right\|<\varepsilon\right\}$ e dado que $\mathcal{U}$ é um filtro obtemos que $\left\{i \in I \mid\left\|x_{i}\right\|<\varepsilon\right\} \in \mathcal{U}$. Como $\varepsilon>0$ é arbitrário obtemos que $\lim _{\mathcal{U}}\left\|x_{i}\right\|=0$.

Definimos sobre $l_{\infty}\left(I, X_{i}\right)$ a seguinte seminorma,

$$
\left\|\left\{x_{i}\right\}_{i \in I}\right\|_{\mathcal{U}}=\lim _{\mathcal{U}}\left\|x_{i}\right\|
$$

Por definição do subespaço $N_{\mathcal{U}}$, obtemos que dita seminorma determina uma norma sobre o espaço quociente $l_{\infty}\left(I, X_{i}\right) / N_{\mathcal{U}}$ e denotamos os elementos do quociente por $\left(x_{i}\right)_{\mathcal{U}}$.

Definição $3.2 O \mathcal{U}$-ultraproduto ou ultraproduto da familia $\left\{X_{i}\right\}_{i \in I}$, onde cada $X_{i}$ é um espaço normado, com respeito ao ultrafiltro $\mathcal{U}$ sobre $I$ é o espaço quociente normado

$$
\left(X_{i}\right)_{\mathcal{U}}=l_{\infty}\left(I, X_{i}\right) / N_{\mathcal{U}}
$$

com norma $\left\|\left(x_{i}\right)_{\mathcal{U}}\right\|=\lim _{\mathcal{U}}\left\|x_{i}\right\|$.

Notemos que se $X_{i}$ é um subespaço normado de $Y_{i}$, para $i \in I$, então $\left(X_{i}\right)_{\mathcal{U}} \subseteq\left(Y_{i}\right)_{\mathcal{U}}$. Se para cada $i \in I, X_{i}=X$, onde $X$ é um espaço normado, dizemos que $\left(X_{i}\right)_{\mathcal{U}}=X_{\mathcal{U}}$, é a ultrapotência de $X$ com respeito ao ultrafiltro $\mathcal{U}$. Nesse caso, para cada $x \in X$ denotamos por $\check{x}$ o elemento $\left(x_{i}\right)_{\mathcal{U}} \in X_{\mathcal{U}}$ tal que $x_{i}=x$ par todo $i \in I$. Escrevemos $\check{X}=\{\check{x}: x \in X\}$.

Lema 3.4 Seja $X$ um espaço normado de dimensão finita. Então $X_{\mathcal{U}}=\check{X}$.

\section{Demonstração:}

Mostremos que $X_{\mathcal{U}} \subseteq \check{X}$. Seja $\left(x_{i}\right)_{\mathcal{U}} \in X_{\mathcal{U}}$ e $\left\{x_{i}\right\}_{i \in I} \in\left(x_{i}\right)_{\mathcal{U}}$. A família $\left\{x_{i}\right\}_{i \in I}$ é limitada, portanto está contida em uma bola fechada limitada de $X$ e como o espaço é de dimensão finita, dita bola é compacta e pelo Lema 3.3, existe $y$ na bola tal que $\lim _{\mathcal{U}} x_{i}=y$, isto é, para cada $\varepsilon>0$ o conjunto $\left\{i \in I \mid x_{i} \in B(y, \varepsilon)\right\} \in \mathcal{U}$, ou seja, $\left\{i \in I \mid\left\|x_{i}-y\right\|<\varepsilon\right\} \in \mathcal{U}$. Donde, $\left\{i \in I \mid\left\|x_{i}-y_{i}\right\|<\varepsilon\right\} \in \mathcal{U}$ com $y_{i}=y$ para todo $i \in I$. Desse modo a família $\left\{x_{i}-y_{i}\right\}_{i \in I}$ é tal que $\lim _{\mathcal{U}}\left\|x_{i}-y_{i}\right\|=0$, assim $\left\{x_{i}-y_{i}\right\}_{i \in I} \in N_{\mathcal{U}}$. Obtemos então que $\left\{x_{i}\right\}_{i \in I}$ e $\left\{y_{i}\right\}_{i \in I}$ são equivalentes módulo $N_{\mathcal{U}}$, isto é, $\left(x_{i}\right)_{\mathcal{U}}=\check{y}=\left(y_{i}\right)_{\mathcal{U}} \in \check{X}$.

Seja $T: X \rightarrow X_{\mathcal{U}}$ dada por $x \mapsto \check{x}$. Então $T$ é uma isometria linear, pois $\|T(x)\|=$ $\left\|\left(x_{i}\right)_{\mathcal{U}}\right\|=\lim _{\mathcal{U}}\|x\|=\|x\|$. Por definição, $T$ é linear e como é uma isometria é injetora. Daí, $X$ é isomorfo (isométrico) com $\check{X}$ que é um subespaço de $X_{\mathcal{U}}$. Quando $X$ é de dimensão finita, $X \cong X_{\mathcal{U}}$ e pelo Lema $3.4, X \cong X_{\mathcal{U}}=\check{X}$.

Corolário 3.1 Seja $\left\{X_{i}\right\}_{i \in I}$ uma familia de espaços de Hilbert sobre $\mathbb{F}$ tal que existe um número natural $n$ com $\operatorname{dim}\left(X_{i}\right) \leq n$ para todo $i \in I$. Então $\operatorname{dim}\left(\left(X_{i}\right)_{\mathcal{U}}\right) \leq n$.

\section{Demonstração:}

Seja $X$ um $\mathbb{F}$ espaço vetorial de dimensão $n$ com produto interno. Para cada $i \in I$, existe uma isometria linear $T_{i}: X_{i} \rightarrow X$. Então $G:\left(X_{i}\right)_{\mathcal{U}} \rightarrow X_{\mathcal{U}}$ definida por $\left(x_{i}\right)_{\mathcal{U}} \mapsto\left(T_{i}\left(x_{i}\right)\right)_{\mathcal{U}}$. 
A aplicação $G$ é linear pois cada $T_{i}$ é linear e $G$ está bem definida já que se $\left\{x_{i}\right\}_{i \in I}$ e $\left\{y_{i}\right\}_{i \in I}$ em $l_{\infty}\left(I, X_{i}\right)$ são tais que $\lim _{\mathcal{U}}\left\|x_{i}-y_{i}\right\|=0$, então para $\left\{T_{i}\left(x_{i}\right)\right\}_{i \in I}$ e $\left\{T_{i}\left(y_{i}\right)\right\}_{i \in I}$ temos que

$$
\lim _{\mathcal{U}}\left\|T_{i}\left(x_{i}\right)-T_{i}\left(y_{i}\right)\right\|=\lim _{\mathcal{U}}\left\|T_{i}\left(x_{i}-y_{i}\right)\right\|=\lim _{\mathcal{U}}\left\|x_{i}-y_{i}\right\|=0
$$

$\operatorname{assim} G\left(\left(x_{i}\right)_{\mathcal{U}}\right)=G\left(\left(y_{i}\right)_{\mathcal{U}}\right)$. A aplicação $G$ é uma isometria pois, dado $\left(x_{i}\right)_{\mathcal{U}} \in\left(X_{i}\right)_{\mathcal{U}}$ obtemos que, $\left\|G\left(\left(x_{i}\right)_{\mathcal{U}}\right)\right\|=\left\|\left(T_{i}\left(x_{i}\right)\right)_{\mathcal{U}}\right\|=\lim _{\mathcal{U}}\left\|T_{i}\left(x_{i}\right)\right\|=\lim _{\mathcal{U}}\left\|x_{i}\right\|=\left\|\left(x_{i}\right)_{\mathcal{U}}\right\|$. Então $G:\left(X_{i}\right)_{\mathcal{U}} \rightarrow$ $X_{\mathcal{U}}$ é uma isometria linear, pelo Lema $3.4, \operatorname{dim} X_{\mathcal{U}}=n$ e como $G$ é injetora $\operatorname{dim}\left(X_{i}\right)_{\mathcal{U}} \leq n$.

Seja $\left\{A_{i}\right\}_{i \in I}$ uma família de $\mathbb{F}$-álgebras normadas. Temos que $\left(A_{i}\right)_{\mathcal{U}}$ é um espaço normado, definimos um produto sobre $\left(A_{i}\right)_{\mathcal{U}}$ tal que este espaço normado seja uma $\mathbb{F}$-álgebra normada. Para $\left(x_{i}\right)_{\mathcal{U}}$ e $\left(y_{i}\right)_{\mathcal{U}}$ em $\left(A_{i}\right)_{\mathcal{U}}$ definimos

$$
\left(x_{i}\right)_{\mathcal{U}} \cdot\left(y_{i}\right)_{\mathcal{U}}=\left(x_{i} y_{i}\right)_{\mathcal{U}}
$$

Este produto está bem definido, já que se $\left(x_{i}^{\prime}\right)_{\mathcal{U}}=\left(x_{i}\right)_{\mathcal{U}}$ e $\left(y_{i}^{\prime}\right)_{\mathcal{U}}=\left(y_{i}\right)_{\mathcal{U}}$, então $\left\|x_{i} y_{i}-x_{i}^{\prime} y_{i}^{\prime}\right\|=$ $\left\|x_{i} y_{i}-x_{i}^{\prime} y_{i}+x_{i}^{\prime} y_{i}-x_{i}^{\prime} y_{i}^{\prime}\right\|=\left\|\left(x_{i}-x_{i}^{\prime}\right) y_{i}+x_{i}^{\prime}\left(y_{i}-y_{i}^{\prime}\right)\right\| \leq\left\|x_{i}-x_{i}^{\prime}\right\|\left\|y_{i}\right\|+\left\|x_{i}^{\prime}\right\|\left\|y_{i}-y_{i}^{\prime}\right\|$, donde $\lim _{\mathcal{U}}\left\|x_{i} y_{i}-x_{i}^{\prime} y_{i}^{\prime}\right\|=0$ e $\operatorname{assim}\left(x_{i} y_{i}\right)_{\mathcal{U}}=\left(x_{i}^{\prime} y_{i}^{\prime}\right)_{\mathcal{U}}$ o que mostra que o produto está bem definido.

Como $\left\|\left(x_{i}\right)_{\mathcal{U}} \cdot\left(y_{i}\right)_{\mathcal{U}}\right\|=\left\|\left(x_{i} y_{i}\right)_{\mathcal{U}}\right\|=\lim _{\mathcal{U}}\left\|x_{i} y_{i}\right\|$ e dado que $\left\|x_{i} y_{i}\right\| \leq\left\|x_{i}\right\|\left\|y_{i}\right\|$, então $\lim _{\mathcal{U}}\left\|x_{i} y_{i}\right\| \leq \lim _{\mathcal{U}}\left\|x_{i}\right\| \lim _{\mathcal{U}}\left\|y_{i}\right\|$. Portanto,

$$
\left\|\left(x_{i}\right)_{\mathcal{U}} \cdot\left(y_{i}\right)_{\mathcal{U}}\right\| \leq\left\|\left(x_{i}\right)_{\mathcal{U}}\right\|\left\|\left(y_{i}\right)_{\mathcal{U}}\right\|
$$

Assim, $\left(A_{i}\right)_{\mathcal{U}}$ é uma $\mathbb{F}$-álgebra normada.

Se $\left\{A_{i}\right\}_{i \in I}$ for uma família de $\mathbb{F}$-álgebras absolutamente valuadas obtemos, com este produto, que $\left(A_{i}\right)_{\mathcal{U}}$ é uma $\mathbb{F}$-álgebra absolutamente valuada. Quando $A_{i}=A$ para todo $i \in I$ e $A$ é absolutamente valuada obtemos que a ultrapotência de $A$ com respeito a $\mathcal{U}, A_{\mathcal{U}}$, é uma $\mathbb{F}$-álgebra absolutamente valuada e como $A$ é isométrico com $\check{A}$, podemos considerar $A$ como uma subálgebra de $A_{\mathcal{U}}$.

Corolário 3.2 Seja $\left\{A_{i}\right\}_{i \in I}$ uma familia de $\mathbb{R}$-álgebras absolutamente valuadas de dimensão finita. Então $\left(A_{i}\right)_{\mathcal{U}}$ é uma $\mathbb{R}$-álgebra absolutamente valuada de dimensão finita, com $\operatorname{dim}\left(A_{i}\right)_{\mathcal{U}} \leq \max \left\{\operatorname{dim}\left(A_{i}\right) \mid i \in I\right\}$ e a dimensão é menor ou igual a 8.

\section{Demonstração:}

Temos pelo Teorema 1.6 que $\operatorname{dim}\left(A_{i}\right) \leq 8$ para todo $i \in I$, e que a norma está induzida por um produto interno. Então, $\left\{A_{i}\right\}_{i \in I}$ é uma família de espaços de Hilbert e pelo Corolário 3.1, $\operatorname{dim}\left(A_{i}\right)_{\mathcal{U}} \leq 8$.

Teorema 3.1 Seja $\left\{A_{i}\right\}_{i \in I}$ uma família de $\mathbb{R}$-álgebras absolutamente valuadas e algébricas. Então $\left(A_{i}\right)_{\mathcal{U}}$ é uma $\mathbb{R}$-álgebra absolutamente valuada algébrica e

$$
\operatorname{deg}\left(A_{i}\right)_{\mathcal{U}} \leq \max \left\{\operatorname{deg}\left(A_{i}\right) \mid i \in I\right\}
$$

Como consequência a ultrapotência, $A_{\mathcal{U}}$, de uma $\mathbb{R}$-álgebra $A$ algébrica e absolutamente valuada, é uma $\mathbb{R}$-álgebra algébrica, absolutamente valuada e $\operatorname{deg}\left(A_{\mathcal{U}}\right)=\operatorname{deg}(A)$.

\section{Demonstração:}

Pelo comentário que precede ao Corolário 3.2 , temos que $\left(A_{i}\right)_{\mathcal{U}}$ é uma $\mathbb{R}$-álgebra absoluta- 
mente valuada. Seja $\left(a_{i}\right)_{\mathcal{U}}$ em $\left(A_{i}\right)_{\mathcal{U}}$, mostremos que a subálgebra gerada por $\left(a_{i}\right)_{\mathcal{U}}$ é de dimensão finita. Como $A_{i}$ é algébrica, $A_{i}\left(a_{i}\right)$ é de dimensão finita e pelo Corolário $3.2,\left(A_{i}\left(a_{i}\right)\right) \mathcal{U}$ é de dimensão finita com $\operatorname{dim}\left(A_{i}\left(a_{i}\right)\right)_{\mathcal{U}} \leq \max \left\{\operatorname{dim}\left(A_{i}\left(a_{i}\right)\right) \mid i \in I\right\}$. Agora, $\left(A_{i}\left(a_{i}\right)\right)_{\mathcal{U}}$ é uma subálgebra de $\left(A_{i}\right)_{\mathcal{U}}$ e $\left(a_{i}\right)_{\mathcal{U}} \in\left(A_{i}\left(a_{i}\right)\right)_{\mathcal{U}}$, então a subálgebra gerada por $\left(a_{i}\right)_{\mathcal{U}}$ é de dimensão finita. Assim, $\left(A_{i}\right)_{\mathcal{U}}$ é algébrica. Temos assim que $\left(A_{i}\right)_{\mathcal{U}}$ é absolutamente valuada e algébrica, portanto de grau limitado. Agora,

$$
\operatorname{dim}\left(A_{i}\left(a_{i}\right)\right)_{\mathcal{U}} \leq \max \left\{\operatorname{dim}\left(A_{i}\left(a_{i}\right)\right) \mid i \in I\right\} \leq \max \left\{\operatorname{deg}\left(A_{i}\right) \mid i \in I\right\},
$$

donde $\operatorname{deg}\left(A_{i}\right)_{\mathcal{U}} \leq \max \left\{\operatorname{deg}\left(A_{i}\right) \mid i \in I\right\}$. Quando $A_{i}=A$ para todo $i \in I, \operatorname{deg}\left(A_{\mathcal{U}}\right) \leq \operatorname{deg}(A)$ e como podemos considerar $A$ como subálgebra de $A_{\mathcal{U}} \operatorname{deg}(A) \leq \operatorname{deg}\left(A_{\mathcal{U}}\right)$. Desse modo $\operatorname{deg}\left(A_{\mathcal{U}}\right)=\operatorname{deg}(A)$.

Para mostrar a seguinte proposição definimos primeiro o conceito de $\mathbb{F}$-álgebra livre não associativa (sem unidade) sobre um conjunto não vazio $X$ que denotaremos por $\mathbb{F}\langle X\rangle$. Podemos dizer que $X$ é um conjunto de indeterminadas e assim os elementos de $\mathbb{F}\langle X\rangle$ são expressões formais nas indeterminadas de $X$.

Para definir $\mathbb{F}\langle X\rangle$, definimos primeiro o conceito de palavra não associativa nas indeterminadas de $X$.

Definimos as palavras não associativas de comprimento $n$, com $n$ inteiro positivo, indutivamente da seguinte maneira:

i) As palavras de comprimento 1 são os elementos de $X$;

ii) As palavras de comprimento 2 são todas as expressões formais $x y \operatorname{com} x, y \in X$;

iii) Para $n>2$, as palavras de comprimento $n$ são obtidas por justaposição de palavras de menor comprimento, das seguintes formas:

$$
x(u),(u) x,(v)(w)
$$

com $x \in X, u$ palavra de comprimento $n-1$, e $v$ e $w$ palavras de comprimento $\leq n-2$ tal que a soma dos comprimentos de $v$ e $w$ seja $n$.

Uma palavra de comprimento $n$ chama-se também monômio de grau $n$. $\mathbb{F}\langle X\rangle$ é a $\mathbb{F}$-álgebra tal que o conjunto de palavras definidas acima formam uma base de $\mathbb{F}\langle X\rangle$ como $\mathbb{F}$-espaço vetorial e o produto de duas palavra é uma outra palavra obtida por justaposição da seguinte maneira:

$$
x \cdot y=x y, \quad x \cdot u=x(u), \quad u \cdot x=(u) x, \quad u \cdot v=(u)(v),
$$

para todo $x \in X$, e $u$ e $v$ palavras de comprimento $\geq 2$.

Temos também a propriedade universal das álgebras livres não associativas: seja $A$ uma $\mathbb{F}$-álgebra, e $\varphi: X \rightarrow A$ uma aplicação qualquer. Então existe um único homomorfismo de $\mathbb{F}$-álgebras $\psi: \mathbb{F}\langle X\rangle \rightarrow A$ tal que $\psi(x)=\varphi(x)$ para todo $x \in X$, isto é, a aplicação $\varphi$ pode ser estendida de uma única forma a um homomorfismo de $\mathbb{F}\langle X\rangle$ em $A$.

A aplicação $\psi$ é definida por indução sobre o comprimento dos elementos de $\mathbb{F}\langle X\rangle$ do seguinte modo. Se $x \in X$, então obviamente definimos $\psi(x)=\varphi(x)$. Seja agora $n>1 \mathrm{e}$ suponhamos por indução definida $\psi$ para palavras de comprimento menor do que $n$. Seja $u$ uma palavra de comprimento $n$. Então existem duas palavras $u, w$ (que são únicas) tal que $u=v \cdot w$. Definimos $\psi(u)=\psi(v) \psi(w)$. Finalmente, se $p=\sum_{i-1}^{n} \lambda_{i} u_{i} \in \mathbb{F}\langle X\rangle \operatorname{com} \lambda_{i} \in \mathbb{F} \mathrm{e}$ $u_{i}$ palavras, $\psi(p)=\sum_{i-1}^{n} \lambda_{i} \psi\left(u_{i}\right)$. 
Proposição 3.2 Sejam A uma $\mathbb{F}$-álgebra normada e $n \in \mathbb{N}$. Então

$$
A_{n}=\{a \in A \mid \operatorname{dim}(A(a)) \leq n\}
$$

é fechado em A.

\section{Demonstração:}

Seja $\mathbb{F}\langle x\rangle$ a $\mathbb{F}$-álgebra universal livre não associativa gerada por $\{x\}$. Sejam $a \in A$ e $p(x) \in$ $\mathbb{F}\langle x\rangle$. Então $p(a)$ representa a imagem de $p$ no único homomorfismo de $\mathbb{F}$-álgebras de $\mathbb{F}\langle x\rangle$ em $A$ que transforma $x$ em $a$. Notamos que $p(a)$ pode ser obtido, substituindo em $p$ a variável $x$ por $a$.

Como a soma, o produto por escalar e o produto em $A$ são aplicações contínuas, a função $a \mapsto p(a)$ de $A$ em $A$ é contínua. Seja $a \in \overline{A_{n}}$ e mostremos que $a \in A_{n}$. Temos que existe uma sequência $\left\{a_{k}\right\}_{k \in \mathbb{N}}$ em $A_{n}$ tal que $\lim _{k \rightarrow \infty} a_{k}=a$. Se $x_{0}, \ldots, x_{n}$ são elementos de $A(a)$, a subálgebra de $A$ gerada por $a$, então existem $p_{0}, \ldots, p_{n}$ em $\mathbb{F}\langle x\rangle$ tais que $p_{i}(a)=x_{i}$, para $0 \leq i \leq n$. O conjunto $\Phi_{k}=\left\{p_{0}\left(a_{k}\right), \ldots, p_{n}\left(a_{k}\right)\right\}$ é linearmente dependente em $A$ para todo $k \in \mathbb{N}$, pois $p_{i}\left(a_{k}\right) \in A\left(a_{k}\right)$ e $\operatorname{dim}\left(A\left(a_{k}\right)\right) \leq n$. Portanto, para cada $k \in \mathbb{N}$ existe $\mathbf{v}_{k}=\left(\lambda_{0, k}, \ldots, \lambda_{n, k}\right)$ em $\mathbb{F}^{n+1}$ tal que $\sum_{i=0}^{n}\left|\lambda_{i, k}\right|=1$ e $\sum_{i=0}^{n} \lambda_{i, k} p_{i}\left(a_{k}\right)=0$. Obtemos assim uma sequência $\left\{\mathbf{v}_{k}\right\}_{k \in \mathbb{N}}$ na esfera unitária de $l_{1}^{n+1}(\mathbb{F})$, onde $l_{1}^{n+1}(\mathbb{F})$ é o espaço vetorial $\mathbb{F}^{n+1}$ com norma $\left\|\left(\alpha_{0}, \ldots, \alpha_{n}\right)\right\|_{1}=\sum_{i=0}^{n}\left|\alpha_{i}\right|$. Dado que dita esfera é compacta, existe uma subsequência $\left\{\mathbf{v}_{k_{j}}\right\}_{j \in \mathbb{N}}$ de $\left\{\mathbf{v}_{k}\right\}_{k \in \mathbb{N}}$ que converge a um ponto $\mathbf{v}=\left(\lambda_{0}, \ldots, \lambda_{n}\right)$ da esfera.

Para cada $i$, a aplicação $a \mapsto p_{i}(a)$ é contínua logo

$$
\lim _{k \rightarrow \infty} p_{i}\left(a_{k}\right)=p_{i}\left(\lim _{k \rightarrow \infty} a_{k}\right)=p_{i}(a)=x_{i},
$$

$\log 0$

$$
\begin{aligned}
0=\lim _{j \rightarrow \infty} \sum_{i=0}^{n} \lambda_{i, k_{j}} p_{i}\left(a_{k_{j}}\right) & =\sum_{i=0}^{n} \lim _{j \rightarrow \infty} \lambda_{i, k_{j}} p_{i}\left(a_{k_{j}}\right)=\sum_{i=0}^{n}\left(\lim _{j \rightarrow \infty} \lambda_{i, k_{j}}\right)\left(\lim _{j \rightarrow \infty} p_{i}\left(a_{k_{j}}\right)\right) \\
& =\sum_{i=0}^{n}\left(\lim _{j \rightarrow \infty} \lambda_{i, k_{j}}\right)\left(p_{i}\left(\lim _{j \rightarrow \infty} a_{k_{j}}\right)\right)=\sum_{i=0}^{n} \lambda_{i} p_{i}(a)=\sum_{i=0}^{n} \lambda_{i} x_{i} .
\end{aligned}
$$

Daí, o conjunto $\left\{x_{1}, \ldots, x_{n+1}\right\}$ é linearmente dependente em $A(a)$ e consequentemente $\operatorname{dim}(A(a)) \leq n$

\section{$3.2 \quad$ O Resultado}

Corolário 3.3 Seja A uma $\mathbb{F}$-álgebra algébrica e normada de grau limitado. Então o conjunto $B=\{a \in A \mid \operatorname{dim}(A(a))=\operatorname{deg}(A)\}$ é aberto em $A$.

\section{Demonstração:}

Seja $n=\operatorname{deg}(A)$. Pela Proposição $3.2 A_{n-1}=\{a \in A: \operatorname{dim} A(a)<n\}$ é fechado. Assim, $B=A \backslash A_{n-1}$ é aberto.

Corolário 3.4 Seja A uma $\mathbb{R}$-álgebra algébrica e absolutamente valuada. Então existe a $\in A$ tal que $\|a\|=1, \operatorname{dim}(A(a))=\operatorname{deg}(A)$ e a norma de $A$ é Fréchet diferenciável em a. 


\section{Demonstração:}

Como $A$ é algébrica e absolutamente valuada, pelo Teorema 1.6, obtemos que $A$ é de grau limitado $\leq 8$ e pelo Corolário 3.3, $B=\{b \in A \mid \operatorname{dim}(A(b))=\operatorname{deg}(A)\}$ é um conjunto aberto em $A$. Então dado $b \in B$ existe $\delta>0$ tal que $B(b, \delta) \subseteq B$. Pelo Lema 2.2, existe $x \in B(b, \delta) \cap \Omega$. Definimos $a=\frac{x}{\|x\|}$. Temos que $A(a)=A(x)$ e portanto $\operatorname{dim}(A(a))=\operatorname{deg}(A)$. Finalmente, por Teorema 2.15 seque que a norma é Fréchet diferenciável em $a$.

Lembremos que o fato de que um espaço normado seja suave em um elemento não zero é equivalente ao fato de que a norma seja Gateaux diferenciável nesse elemento, portanto se a norma é Fréchet diferenciável em um ponto não zero, o espaço é suave no ponto. O seguinte é o resultado principal desta dissertação.

Teorema 3.2 Seja A uma $\mathbb{R}$-álgebra algébrica e absolutamente valuada. Então $A$ é de dimensão finita.

\section{Demonstração:}

Tal como é mostrado no comentário que precede ao Corolário 3.2, consideramos $A$ como subálgebra da ultrapotência de $A$, identificando $b \in A$ por $\left(b_{n}\right)_{\mathcal{U}} \in A_{\mathcal{U}}$ onde $b_{n}=b$ para todo $n \in N$.

Seja, pelo Corolário 3.4, $a \in A,\|a\|=1$, tal que $A$ é suave em $a$ e $\operatorname{deg}(A)=\operatorname{dim}(A(a))$. Definimos $M=\{x \in A(a) \mid a x=x\}, M$ é um subespaço de $A$ e como a dimensão de $A(a)$ é finita obtemos que $\operatorname{dim}(M)<\infty$. Pelo Teorema 1.6, temos que a restrição da norma de $A$ ao subespaço $M$ provém de um produto interno e pela Proposição 2.4, $A$ é suave em todo elemento de norma um em $M$.

Como $L_{a}: A \rightarrow A, x \mapsto a x$, é tal que $L_{a}(m)=m$ para todo $m \in M$ e $\left\|L_{a}(x)\right\|=\|x\|$ então $\left\|L_{a}\right\|=1$ o que permite aplicar a Proposição $2.3 \operatorname{com} T=L_{a}$, daí existe $\pi: A \rightarrow A$ uma projeção linear contínua tal que $\pi(A)=M$ e $k e r(\pi)$ é $L_{a}$-invariante. Suponhamos que $A$ é de dimensão infinita.

Pelo Corolário 2.1, aA ou $A a$ não é denso em $A$. Podemos supor que $L_{a}(A)=a A$ não é denso. Dado que $A=M \oplus \operatorname{ker}(\pi)$, a restrição de $L_{a}$ a $M$ é a identidade e $k e r(\pi)$ é $L_{a}$-invariante, obtemos que a aplicação $G: \operatorname{ker}(\pi) \rightarrow \operatorname{ker}(\pi), y \mapsto a y$, é isometria linear e $G(k e r(\pi))$ não é denso em $\operatorname{ker}(\pi)$. Então pelo Corolário 2.4 existe uma sequência, $\left\{x_{n}\right\}_{n \in \mathbb{N}}$, em $\operatorname{ker}(\pi) \operatorname{com}$ $\left\|x_{n}\right\|=1$ para todo $n$ tal que a sequência $\left\{a x_{n}-x_{n}\right\}_{n \in \mathbb{N}} \rightarrow 0$.

Agora seja $\mathcal{U}$ um ultrafiltro sobre $\mathbb{N}$, obtido refinando o filtro de Fréchet, e seja também $\beta \in A_{\mathcal{U}}$ dado por $\beta=\left(x_{n}\right)_{\mathcal{U}}$, observemos que $\left\|\left(x_{n}\right)_{\mathcal{U}}\right\|=\lim _{\mathcal{U}}\left\|x_{n}\right\|=1$, assim $\beta$ é elemento de norma um na ultrapotência de $A$. Tendo em consideração que $A$ é uma subálgebra de $A_{\mathcal{U}}$

$$
a \beta=\left(a_{n}\right)_{\mathcal{U}}\left(x_{n}\right)_{\mathcal{U}}=\left(a_{n} x_{n}\right)_{\mathcal{U}}=\left(a x_{n}\right)_{\mathcal{U}}
$$

e como $\left\{a x_{n}-x_{n}\right\}_{n \in \mathbb{N}} \rightarrow 0$ obtemos que para todo $\varepsilon>0\left\{n \in \mathbb{N} \mid\left\|a x_{n}-x_{n}\right\|<\varepsilon\right\} \in \mathcal{U}$ o que implica que $\left(a x_{n}\right)_{\mathcal{U}}=\left(x_{n}\right)_{\mathcal{U}}$, isto é, $a \beta=\beta$ em $A_{\mathcal{U}}$. Pelo Teorema 3.1, $A_{\mathcal{U}}$ é uma $\mathbb{R}$-álgebra algébrica absolutamente valuada e $\operatorname{deg}\left(A_{\mathcal{U}}\right)=\operatorname{deg}(A)$, como $A(a) \subseteq A_{\mathcal{U}}(a)$ e $\operatorname{dim}(A(a))=\operatorname{deg}(A)$ obtemos que $\operatorname{dim}\left(A_{\mathcal{U}}(a)\right) \leq \operatorname{dim}(A(a))$ portanto $A_{\mathcal{U}}(a)=A(a)$, daí $\operatorname{dim}\left(A_{\mathcal{U}}(a)\right)=\operatorname{deg}\left(A_{\mathcal{U}}\right)$ o que implica, pela Proposição 2.5 , que $A_{\mathcal{U}}(\beta)=A_{\mathcal{U}}(a)=A(a)$, portanto $\beta \in A(a)$ e como $a \beta=\beta$, segue que $\beta \in M$.

Finalmente mostremos que $\beta \in \operatorname{ker}(\pi)$ e obteremos assim uma contradição. Como $\beta \in A$, pela definição de ultraproduto (de espaço normado), ter $\beta=\left(x_{n}\right)_{\mathcal{U}}$ é equivalente a ter $\lim _{\mathcal{U}}\left\|\beta-x_{n}\right\|=0$. Dado que $\left\{x_{n}\right\}_{n \in \mathbb{N}} \in \operatorname{ker}(\pi)$, obtemos $\pi(\beta)=0$. Assim $\beta \in M \cap k e r(\pi)$, isto é, $\beta=0$ o que é absurdo pela definição de $\beta$. Então $A$ é de dimensão finita. 
Observemos que pelo Teorema $1.6, A$ é isótopa com $\mathbb{R}, \mathbb{C}, \mathbb{H}$ ou $\mathbb{D}$ e que a norma está induzida por um produto interno. 


\section{Referências Bibliográficas}

[1] Albert, A.A. Absolute Valued Real Algebras. Ann. of Math. Vol. 48, 1947 495-501. xiii, 5,12

[2] Albert, A.A. Absolute-Valued Algebraic Algebras. Bull. Amer. Math. Soc. Vol. 55. 1949. 763-768. xiii, 19

[3] Palacios, A. R. One Sided Division Absolute Valued Algebras. Publ. Mat. 36, 1992 925-954.

[4] Albiac, F; Kalton, N.J. Topics in Banach Space Theory. Springer. New York, 2006.

[5] Becerra, J. G.; Moreno, Antonio. A.; Rodríguez, Ángel. P. Absolute Valuable Banach Spaces. Illinois J. Math. 49, 2005, 121-138. 25, 27

[6] Berberian, S.K. Lectures in Functional Analysis and Operator Theory. Springer-Verlag, New York-Heidelberg, 1974. 35

[7] Calderón, A.; Kaidi, A.; Martín, C.; Morales, A.; Ramírez, M.; Rochdi, A. Finite Dimensional Absolute Valued Algebras. Israel J. Math. 184, 2011 193-220.

[8] Elduque, A.; Pérez J.M. Infinite Dimensional Quadratic Forms Admiting Composition. Proc. Amer. Math. Soc. 125, 1997 2207-2216. 5

[9] El-Amin, K; Ramírez, M.I; Palacios, A.R. Absolute Valued Algebraic Algebras Are Finete Dimensional. J. Algebra 195, 1997 295-307. xiv, 29, 30, 35, 41, 47, 49, 51

[10] Fontes, F.G. Espectro de una Isometria Lineal. Carta informativa SMM, No. 51, 2007 1-4. 35, 39, 41

[11] Heinrich,S. Ultraproducts in Banach Space Theory. J. Reine Angew. Math. 313, 1980, 72-104. 51

[12] Herstein, I.N. Topics in algebra. Ginn and company, London, 1964. 15

[13] Ingelstam, L. Non-associative Normed Algebras and Hurwitz'problem. Ark.Mat. 5, 1964 231-238.

[14] Jacobson, N. Composition algebras and their automorphisms. Rend. Circ. Mat. 7, 1958 55-80. 12

[15] Jacobson, N. Basic Algebra. I. W. H. Freeman and Co. San Francisco, Calif, 1974.

[16] Junior, H.G. O Teorema de Frobenius para Álgebras não Associativas. Dissertação, 1985. xiii, $6,12,41$ 
[17] Kaplansky, I. Infinite Dimensional Quadratic Forms Admitting Composition. Proc. Amer. Math. Soc. 36, 1953 956-960. 15

xiii, 6

[18] Kreyszig,E. Introduction Functional Analysis with Applications. Wiley Classics Library. New York, 1989. 18, 29, 35

[19] Ma,T.W. Classical Analysis on Normed Spaces. Word Scientific Publishing Co. New Jersey, 1995. 41

[20] MacCrimmon, K. A Taste of Jordan Algebras. Springer-Verlag. New York, 2004. 6

[21] Megginson, R.E. An Introduction to Banach Space Theory. Springer-Verlag. New York, 1998. 41,48

[22] Myung, H.CH. Non-Unital Composition Algebras. Lectures Notes Series, 22, 1994 viii1085

[23] Nelson, G.C. Compactness, Ultralimits, Ultraproducts and Maximal Ideals. Department of Mathematics, University of Iowa, Iowa City, Iowa 52242. 51, 54

[24] Oneto, Angel. Alternative Real Division Algebras of Finite Dimension. Divulg. Mat. 10, 2002 161-169. 15

[25] Palacios, A.R. One Sided Division Absolute Valued Algebras. Publ. Mat. 36, 1992 925-954. 35, 39

[26] Roman, S. Advanced Linear Algebra. Springer. New York, 2005.

[27] Schafer, R.D. An introduction to Nonassociative Algebras. Academic Press. New York, 1966. 6

[28] Schoenberg, I. J. A remark on M. M. Day's characterization of inner product spaces and a conjeture of L. M. Blumenthal. Proc. Amer. Math. Soc. 3, 1952 961-964. 14

[29] Slinko, A. 1, 2, 4, 8,... What comes next?. Extracta Math. 19, 2004 155-1661. 3

[30] Urbanik, K; Wright, F.B. Absolute Valued Algebras. Proc. Amer. Math. Soc. 11, 1960 861-866. xiii, xiv, 25, 26, 27

[31] Wright, F.B. Absolute Valued Algebras. Proc. Nat. Acad. Sci. U.S.A. 39, 1953 330-332. xiii, 12,29 


\section{Índice Remissivo}

Álgebra

algébrica, 1

alternativa, 7

de Banach, 35

de composição, 6

de grau limitado, 48

livre não associativa, 54

normada, 1

quadrática, 6

associador de uma, 6, 15

central, 15

centro de uma, 15

comutador de uma, 15

núcleo de uma, 15

Álgebra Absolutamente Valuada (a.a.v), 1

Álgebras

de Hurwitz, 9

isótopas, 5

Completamento de um espaço, 30

Complexificação de uma álgebra, 12

Conjunto J-ortogonal, 19

Derivada

de Fréchet, 41

de Gateaux, 42

Diferenciabilidade da norma, 46

Divisor topológico de zero, 37

Duplicação de Cayley-Dickson, 8

Espaço completo, 30

Espaço métrico, 29

Espaço ultracompleto, 51

Espectro de $x \in A, 36$

Filtro , 49

de Fréchet, 50

Forma quadrática não degenerada, 6

Função polinomial , 44

Identidade de Moufang, 7

Involução , 8 estândar, 7

Normas equivalentes, 18

Octônios, 3

Octônios

tabela do produto dos, 3

Polinômio Homogêneo, 44

Produto de Jordan, 19

Quatérnios , 2

duplicação dos, 3

tabela do produto dos, 2

Radical de uma forma quadrática, 6

Raiz quadrada, 19

Regras do produto de Cayley-Dickson, 9

Resolvente de $x \in A, 36$

Sequência de Cauchy, 29

Suavidade de $X$ em $x \neq 0,47$

Teorema

de Albert, 17

de Frobenius, 15

de Hahn-Banach, 35

de K. Urbanik e F. Wright, 25

Ultrafiltro, 49

Ultrapotência de $X, 52$

Ultraproduto de espaços normados, 52 